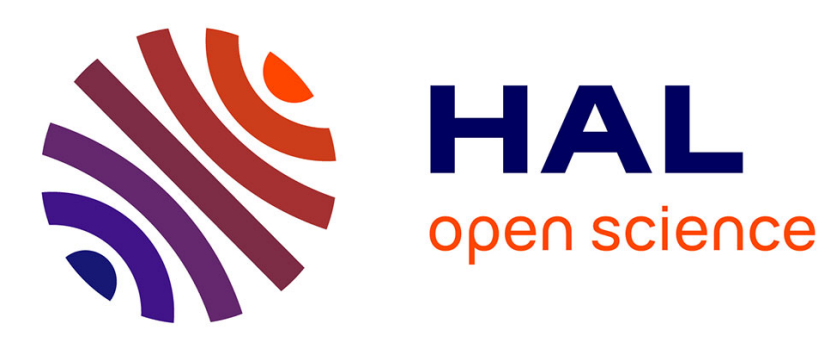

\title{
Direct simulation of rigid particles in a viscoelastic fluid
}

Astrid Decoene, Sébastien Martin, Bertrand Maury

\section{To cite this version:}

Astrid Decoene, Sébastien Martin, Bertrand Maury. Direct simulation of rigid particles in a viscoelastic fluid. 2018. hal-01456089v2

\section{HAL Id: hal-01456089 \\ https://hal.science/hal-01456089v2}

Preprint submitted on 4 Jun 2018

HAL is a multi-disciplinary open access archive for the deposit and dissemination of scientific research documents, whether they are published or not. The documents may come from teaching and research institutions in France or abroad, or from public or private research centers.
L'archive ouverte pluridisciplinaire HAL, est destinée au dépôt et à la diffusion de documents scientifiques de niveau recherche, publiés ou non, émanant des établissements d'enseignement et de recherche français ou étrangers, des laboratoires publics ou privés. 


\title{
Direct simulation of rigid particles in a viscoelastic fluid
}

\author{
Astrid Decoene*, Sébastien Martin† Bertrand Maury ${ }^{\ddagger}$
}

June 4,2018

\begin{abstract}
In this paper, we present a numerical method which performs the direct simulation of $2 \mathrm{D}$ viscoelastic suspensions. Objectives. Interactions between viscoelastic fluids of Oldroyd type, including inertial effects, and a large number of rigid disks or ellipsoids are addressed. Method. The numerical method is built upon a fictitious domain approach which consists in defining the fluid-structure problem over the whole domain (fluid+solid), see e.g. 19. In the Newtonian case 33, 39, the resulting variational formulation, although classical, uses non-standard functional spaces which include the rigidity constraints. From the numerical point of view, these constraints can be addressed by penalty methods, leading to the possible use of standard finite elements solvers with fixed structured meshes. We show how this approach can be adapted to viscoelastic fluids of Oldroyd type. Results. Two types of configurations are investigated in dilute and dense regimes: sedimentation of circular particles and shear flows with ellipsoidal particles.
\end{abstract}

Keywords. viscoelastic fluid, Oldroyd model, fluid-structure interaction, fictitious domain, penalty method, characteristics method, dense suspensions

MSC. 76D03, 76A10, 35Q35

\section{Introduction}

The motion of rigid bodies interacting in a viscoelastic fluid is a well-studied problem from experimental, theoretical and numerical points of view, with a wide range of practical applications such as the study of muds, slurries or polymer melts. The interactions of viscosity, elasticity and multi-body particle inclusions give rise to some complex unexpected phenomena: particle migration, alignment and aggregation of particles, complex rheology of suspensions, etc. Single-particle dynamics, binary interactions and multi-particle systems are discussed in particular in [13.

The simulation of mixtures composed of a large number of rigid entities immerged in a bulk viscoelastic fluid has been the subject of intensive studies. Numerical strategies have been developped in order to incorporate the main features of those complex flows at lower costs: on the one hand, so-called direct simulations aim at solving the fundamental equations of the dynamics at the most precise scale, with numerical algorithms that are proven to be consistent, stable and efficient. On the other hand, approximate simulations may rely on approximations of different kinds leading to an effective simplification of the fundamental equations, such as coarse evaluation of hydrodynamic interactions between the particles thus avoiding the computations related to the fluid dynamics. In this case the underlying equations at the continuous level may not be so clear even if the general dynamics are inspired by the fundamental equations of the coupled problem.

Among the approximate numerical methods, the most popular method is perhaps the so-called "Stokesian dynamics" method that has been introduced by Brady \& Bossis [6, 7. and used primarily for non-equilibrium suspensions. It is based on the determination of both hydrodynamic interactions between the entities inside the fluid and the microstrucure of the suspension. Indeed, in the case of non-Brownian rigid circular particles in a Stokes bulk flow at low Reynolds

\footnotetext{
*Université Paris Sud, Laboratoire de mathématiques d'Orsay (CNRS-UMR 8628), Bâtiment 307, 91405 Orsay cedex, France, astrid.decoene@math.u-psud.fr

†Université Paris Descartes, Laboratoire MAP5 (CNRS UMR 8145), 45 rue des Saints-Pères, 75270 Paris cedex 06, France, sebastien.martin@parisdescartes.fr

¥Université Paris Sud, Laboratoire de mathématiques d’Orsay (CNRS-UMR 8628), Bâtiment 307, 91405 Orsay cedex, France, bertrand.maury@math.u-psud.fr
} 
number, the particles velocity linearly relates to the hydrodynamic forces, see Happel \& Brenner [23] and Brenner \& O'Neil [8], which results in a resistance or mobility formulation that relates the hydrodynamic forces exerted on the particles to the instantaneous velocity of each particle ; the main difficulty consists in building the associated resistance or mobility matrix. In the context of the Stokesian dynamics, computational efficiency of the method is directly related to a pairwise assumption of the interactions: although not fully justified from a theoretical standpoint, this assumption leads to reasonable computational costs and has been checked to provide good agreement with experiments. The Stokesian dynamics method has been extended to viscoelastic fluids by [4] as elastic effects are incorporated by directly calculating particle-dumbbell interactions. Starting from the correspondence principle, the Stokesian dynamics simulation method is also extended in 51 to take into account the viscoelasticity of the suspending fluid at least when the Weissenberg number is small.

In this paper we focus on direct numerical methods that are based on the straight resolution of fundamental equations. Numerical methods to incorporate the fluid-structure issue in direct simulations can be divided into two main classes:

1. the first class of methods relies on moving meshes adapted to the movement of the rigid particles. Remeshing of the fluid domain is needed but it can be coupled to mesh adaptation techniques in order to limit the computational costs. Interestingly in the case of near-contact situations mesh refinement techniques provide a good accuracy in the description of the lubrication forces. However the case of dense suspensions may be delicate because of the many near-contact situations that need to be handled. This was used in particular in [29, 28, 35, 43].

2. the second class of methods rely on the so-called fictitious domain approach, which consists in in extending the time-varying fluid domain into a fixed domain including both the fluid domain and the rigid domain associated to the particles. Rigidity constraints can be tackled in two ways:

- the rigid motion may be enforced using a Lagrange multiplier approach, see e.g. [18, 19, 47, 53, in that case, not only the fluid solver needs to be used iteratively at each time step, but also the number of degrees of freedom may become significant in the case of dense suspensions.

- the rigid constraint may be imposed by a penalty method, see e.g. [1, 48, 33]: in that case, direct solvers can be used at each time step.

Although those methods focused on Newtonian bulk fluids, extensions to viscoelastic fluids were attempted. In particular, particle migration of one or two disks/spheres in a shear viscoelastic flow was numerically investigated in [30, 10, in 2D, in [14 in 3D, by means of direct simulation based upon ALE techniques, and also in [4] by combining a distributed Lagrange multiplier approach with finite volume solvers. The sedimentation of particles in a viscoelastic fluid was studied in 2D in [16, 31] by using ALE methods, in [52, 54, 22, by using a fictitious domain approach with Lagrange multipliers, and in 20] in 3D by combining lattice-Boltzmann methods (for the numerical treatment of the Stokes operator), finite volume methods (for the numerical treatment of the transport of the elastic stress of the fluid) and immersed boundary method (for the fluid-structure interaction). Two-dimensional shear flows in bi-periodic domains, described by Lees-Edwards boundary conditions were also investigated in 32 by using a fictitious domain approach with Lagrange multipliers. The rheology of dilute suspensions was investigated in [46 in 2D by using the same techniques as in 31 .

However, in all the above contributions the number of particles in the viscoelastic fluid is very limited, with less than 8 particles, except in 52 in which the fluidization of 102 particles in a two-dimensional bed is addressed. In this paper, the fictitious domain approach is performed with a penalty method and a splitting procedure, enlightening some specific advantages when targeting long time behavior of dense suspensions: in particular no mesh adaptation or remeshing technique is required; standard finite element solvers can be used; each time step roughly requires the resolution of two linear problems, namely a single Stokes problem with a matrix profile which matches the matrix profile of the unconstrained problem, and a linear transport problem associated to the constitutive Oldroyd model that describes the elastic effects. These advantages allow us to perform direct simulations of flows including a large number of rigid entities, from dilute to dense suspensions, over long time periods. Rheological properties of the combination between rigid entities and viscoelastic fluids can be numerically derived.

The paper is organized as follows: in Section 2, we present the fluid-structure interaction problem. In Section 3 we derive the fictitious domain approach which leads us to a mathematical formulation of the initial problem. In Section 4 we derive the numerical method which is based upon the fictitious domain approach: in particular the coupling strategies between the Stokes solvers and the Oldroyd (constitutive equation) solvers are discussed and, furthermore, the penalty method that deals with the rigidity constraints of the particles is described. In Section 5 numerical results are presented: we address the sedimentation of one particle, two particles and describe the sedimentation of a dense suspension (228 
particles) in both Newtonian and non-Newtonian regimes; we also address shear flows: in particular the influence of the elasticity of the bulk fluid over the Jeffery orbit is investigated and the rheology of dilute to dense suspensions is numerically described.

\section{Modelling of the fluid-particle interaction}

\section{Description of rigid particles}

We consider $\Omega \subset \mathbb{R}^{2}$ a bounded regular domain which is composed of rigid particles embedded in a viscoelastic fluid. We consider a set of $N$ rigid particles and denote by $B(t)=\cup_{i=1, \ldots, N} B_{i}(t)$ the domain occupied by the rigid entities at time $t$. We denote by $\rho_{i}$ the mass density of particle $i$, which is assumed to be constant for the sake of simplicity. We denote by $\mathbf{x}_{i}$ the position of its center-of-mass, $\theta_{i}$ its orientation, $m_{i}=\left|B_{i}(0)\right| \rho_{i}$ its total mass and $J_{i}=\int_{B_{i}(0)} \rho_{i}\left\|\mathbf{x}-\mathbf{x}_{i}(0)\right\|^{2} \mathrm{~d} \mathbf{x}$ its kinematic momentum.

\section{Description of a viscoelastic flow}

As the solid domain is $B(t)$, the fluid domain is $\Omega \backslash \overline{B(t)}$. The behavior of an incompressible fluid is described by Newton's second law, leading to the following set of equations

$$
\begin{array}{rlrl}
\rho_{\mathrm{f}}\left(\partial_{t} \mathbf{u}+(\mathbf{u} \cdot \nabla) \mathbf{u}\right)-\operatorname{div}(\boldsymbol{\Sigma})+\nabla p & =\mathbf{f}_{\mathrm{f}}, & & \text { in } \bigcup_{t \in(0, T)}\{t\} \times(\Omega \backslash \overline{B(t)}), \\
\operatorname{div}(\mathbf{u})=0, & & \text { in } \bigcup_{t \in(0, T)}\{t\} \times(\Omega \backslash \overline{B(t)}),
\end{array}
$$

where $\mathbf{u}:(0, T) \times \Omega \mapsto \mathbb{R}^{2}$ denotes the velocity field, $\boldsymbol{\Sigma}:(0, T) \times \Omega \mapsto \mathbb{R}^{2 \times 2}$ the shear stress tensor, $p:(0, T) \times \Omega \mapsto \mathbb{R}$ is the pressure associated to the incompressibility constraint, $\mathbf{f}_{\mathrm{f}}$ the external forces exerted on the fluid and $\rho_{\mathrm{f}}$ the fluid density (the typical external force is the gravity: $\mathbf{f}_{\mathrm{f}}=-\rho_{\mathrm{f}} g \mathbf{e}_{y}, g$ being the gravity). The Oldroyd model describes the behavior of a viscoelastic fluid. Its principle is built upon a description of the shear stress that interpolates between a purely viscous contribution and a purely elastic contribution. The stress tensor for a viscoelastic fluid of Oldroyd type writes:

$$
\boldsymbol{\Sigma}:=2 \mu(1-r) \mathbb{D}(\mathbf{u})+\boldsymbol{\sigma}, \quad \text { in } \bigcup_{t \in(0, T)}\{t\} \times(\Omega \backslash \overline{B(t)})
$$

where $\boldsymbol{\sigma}: \Omega \mapsto \mathbb{R}^{2 \times 2}$ is a symmetric tensor representing the elastic extra-stress of the fluid. We will denote by $\boldsymbol{S}:=\boldsymbol{\Sigma}-p \mathbb{I}$ the total Cauchy stress tensor. At this point, the set of equations is closed by the Oldroyd model which describes the behavior of the elastic extra-stress with respect to the velocity field:

$$
\lambda\left(\partial_{t} \boldsymbol{\sigma}+(\mathbf{u} \cdot \nabla) \boldsymbol{\sigma}+g_{a}(\nabla \mathbf{u}, \boldsymbol{\sigma})\right)+\boldsymbol{\sigma}-\delta \Delta \boldsymbol{\sigma}=2 r \mu \mathbb{D}(\mathbf{u}), \quad \text { in } \bigcup_{t \in(0, T)}\{t\} \times(\Omega \backslash \overline{B(t)}) .
$$

Here $\lambda$ is the relaxation time that characterizes the elasticity of the fluid whereas $\mu$ is the fluid viscosity; $r \in[0,1]$ is an interpolation parameter: cases $r \in(0,1)$ are often referred as Jeffreys models whereas case $r=1$ is referred as the Maxwell model. Besides, $\mathbb{D}(\mathbf{u})$ (resp. $\mathbb{W}(\mathbf{u})$ ) denotes the symmetric (resp. skew-symmetric) part of the velocity gradient. The function $g_{a},-1 \leq a \leq 1$, is a bilinear mapping related to the total derivative: the function $g_{a}$ is defined as

$$
g_{a}(\nabla \mathbf{u}, \boldsymbol{\sigma})=\mathbb{W}(\mathbf{u}) \cdot \boldsymbol{\sigma}-\boldsymbol{\sigma} \cdot \mathbb{W}(\mathbf{u})-a(\mathbb{D}(\mathbf{u}) \cdot \boldsymbol{\sigma}+\boldsymbol{\sigma} \cdot \mathbb{D}(\mathbf{u})) .
$$

The idea of the objective derivative $\partial_{t}+\mathbf{u} \cdot \nabla+g_{a}(\nabla \mathbf{u}, \cdot)$ is to take the time derivative with respect to a reference frame suitably fixed to the body. Different choices of material frames yield different objective derivatives, see [36. In particular, the material slip parameter $a$ interpolates between the so-called upper-convected model $(a=1)$ and lower-convected model $(a=-1)$. Note that the case $a=0$, which we will favor here, is known as the corotational model. The value of the slippage parameter $a$ may be a matter of debate [5. Parameter $\delta$ is a diffusive parameter of the elastic stress. In standard derivations of Oldroyd model from kinetic models for dilute polymers, this diffusive term is routinely omitted, on the grounds that it is several orders of magnitude smaller than the other terms in the equation. It physically corresponds 
to a center-of-mass diffusion term in the dumbell models and it is in the range of about $10^{-9}$ to $10^{-7}$ when scaled in dimensionless equations.

The standard Oldroyd model (without diffusion: $\delta=0$ ) has been the subject of intensive studies: in particular global existence of weak solutions for any data was proved in 42 in the corotational case only. In the general case, the existence and uniqueness of local strong solutions was shown in [21]; besides, if the fluid is not too elastic and if the data are sufficiently small, then solutions are global. In [45] it is proved that the smallness assumption on the elasticity of the fluid could be relaxed. The diffusive Oldroyd model (with diffusion: $\delta>0$ ) has been studied by a few authors: the existence of global strong solutions in 2D for the Cauchy problem of the diffusive Oldroyd-B model (i.e. $a=+1)$ and uniqueness of the solution among a class of strong solutions was proved in [12. Notice also that other regularizations of the standard Oldroyd model have been studied, see in particular [2].

The stationary version of the Oldroyd model is also of interest in our case, in particular when focusing on the discretizedin-time system. To our knowledge, the main available result is due to Renardy [49] and focuses on the standard model (without diffusion) only: existence and uniqueness of strong solutions is proved under the assumption of small regular data. The method used by Renardy is based on a reformulation of the Oldroyd model as a "Newtonian generalized" fluid: the contribution of the stress $\operatorname{div}(\boldsymbol{\sigma})$ is expressed as an implicit function of the velocity field $\mathbf{u}$ and, then, an iterative scheme is built upon this fully nonlinear system. When including stress diffusion, the mathematical analysis of the model can be approached with a completely different framework, as the diffusive contribution in the stress equation drastically changes the mathematical properties of the system. So far, when considering the diffusive version of the Oldroyd model, it is possible to derive mathematical results by using a classical approach, see 11, based on a weak formulation and on energy estimates: in this case, the diffusive model makes it possible to handle with irregular data and boundary conditions and, in the corotational case, the smallness of the data is not needed anymore.

\section{Initial and boundary value problem and fluid-structure interaction}

The equations are supplemented by initial conditions:

$$
(\mathbf{u}, \boldsymbol{\sigma})(0, \cdot)=\left(\mathbf{u}_{0}, \boldsymbol{\sigma}_{0}\right), \quad \text { in } \Omega \backslash \overline{B(0)},
$$

and boundary conditions located at the frontiers of the domain

$$
\mathbf{u}=\mathbf{0}, \quad \text { on }(0, T) \times \partial \Omega,
$$

and at the interface between the rigid particles and the fluid

$$
\mathbf{u}=\mathcal{U}_{i}, \quad \text { on } \bigcup_{t \in(0, T)}\{t\} \times \partial B_{i}(t),
$$

where $\mathcal{U}_{i}$ denotes the velocity field of particle $i$ associated to a rigid motion. This results from the viscous properties of the fluid which leads to adhering (no-slip) boundary conditions at the fluid-structure interface. As mentioned, $\mathcal{U}_{i}$ stands for the velocity of a rigid particle and, consequently, takes the form

$$
\mathcal{U}_{i}(t, \mathbf{x})=\mathbf{v}_{i}(t)+\omega_{i}(t)\left(\mathbf{x}-\mathbf{x}_{i}\right)^{\perp}
$$

$\mathbf{v}_{i}$ and $\omega_{i}$ being the instantaneous translational and rotational velocities of the particle. The fluid-structure interaction is based upon a coupling which writes:

$$
\begin{aligned}
m_{i} \dot{\mathbf{v}}_{i}(t) & =\int_{B_{i}(t)} \mathbf{f}_{i}(t, \mathbf{x}) \mathrm{d} \mathbf{x}+\int_{\partial B_{i}(t)} \boldsymbol{S}(t, \mathbf{x}) \cdot(-\mathbf{n}(t, \mathbf{x})) \mathrm{d} \gamma(\mathbf{x}) \\
J_{i} \dot{\omega}_{i}(t) & =\int_{B_{i}(t)}\left(\mathbf{x}-\mathbf{x}_{i}(t)\right)^{\perp} \cdot \mathbf{f}_{i}(t, \mathbf{x}) \mathrm{d} \mathbf{x}+\int_{\partial B_{i}(t)}\left(\mathbf{x}-\mathbf{x}_{i}(t)\right)^{\perp} \cdot(\boldsymbol{S}(t, \mathbf{x}) \cdot(-\mathbf{n}(t, \mathbf{x}))) \mathrm{d} \gamma(\mathbf{x})
\end{aligned}
$$

where $\mathbf{f}_{i}$ stands for the external non-hydrodynamic forces applied to particle $i$ (e.g. gravity).

Boundary conditions for the elastic tensor may be of different type. In the diffusive case $\delta>0$ (which we favor for the analysis of the problem), we will consider homogeneous Dirichlet boundary conditions:

$$
\boldsymbol{\sigma}=0, \quad \text { on }(0, T) \times \partial \Omega \text { and } \bigcup_{t \in(0, T)}\{t\} \times \partial B_{i}(t)
$$


In the non-diffusive case $(\delta=0)$, no boundary condition is required on the elastic stress. Actually, denoting by $\mathbf{u}_{d}$ the velocity of the boundaries of the fluid domain (with $\mathbf{u}_{d}=0$ for the fixed boundary $\partial \Omega$ and $\mathbf{u}_{d}$ to be determined for the moving boundary $\partial B$ ), we have $\mathbf{u}-\mathbf{u}_{d}=0$ on the boundaries of the fluid domain. As a consequence characteristics do not enter the fluid domain neither through $\partial \Omega$ nor through $\partial B$.

\section{Fictitious domain approach: mathematical formulation}

From a numerical point of view, we do not want to mesh the fluid domain: the complexity of the mesh due to the presence of inclusions would no allow to use standard solvers, and the computational cost of remeshing could become prohibitive. Therefore, we choose the fictitious domain approach, which has been used for fluid-particle interactions by several authors.

\section{Fictitious domain approach and functional spaces}

We define the following space:

$$
V_{B}:=\left\{\mathbf{u} \in H_{0}^{1}(\Omega): \forall i, \exists\left(\mathbf{v}_{i} \times \omega_{i}\right) \in \mathbb{R}^{2} \times \mathbb{R}, \mathbf{u}(\mathbf{x})=\mathbf{v}_{i}+\omega_{i}\left(\mathbf{x}-\mathbf{x}_{i}\right)^{\perp} \text { in } B_{i}\right\}
$$

In a natural way, if $\mathbf{u}(t, \cdot)$ belongs to $V_{B(t)}$, the velocity field is not only defined in the fluid domain but also on the rigid domain through the relationship:

$$
\mathbf{u}(t, \mathbf{x})=\mathbf{v}_{i}(t)+\omega_{i}(t)\left(\mathbf{x}-\mathbf{x}_{i}(t)\right)^{\perp} .
$$

It can be shown also that, since $V_{B}$ is the space of functions that do not deform $B$, we have also the following property:

$$
V_{B}=\left\{\mathbf{u} \in H_{0}^{1}(\Omega), \mathbb{D}(\mathbf{u})=0 \text { in } B\right\}
$$

Hence the rigid motion can be fully taken into account by extending the velocity field over the whole domain and applying a linear constraint on the velocity field (namely $\mathbb{D}(\mathbf{u})=0$ ). Notice that the knowledge of $\mathbf{u}$ over the whole domain is sufficient to identify the translational and rotational velocities $\mathbf{v}_{i}(t)$ and $\omega_{i}(t)$ (which cannot be prescribed in a straightforward way as they are unknowns) by considering their linear relationships with the velocity field $\mathbf{u}(t, \cdot)$ :

$$
\mathbf{v}_{i}(t)=\mathrm{T}_{i}[\mathbf{u}]:=\frac{\int_{B_{i}(t)} \mathbf{u}(t, \mathbf{x}) \mathrm{d} \mathbf{x}}{\left|B_{i}(t)\right|}, \quad \omega_{i}(t)=\mathrm{R}_{i}[\mathbf{u}]:=\frac{\int_{B_{i}(t)} \mathbf{u}(t, \mathbf{x}) \cdot\left(\mathbf{x}-\mathbf{x}_{i}(t)\right)^{\perp} \mathrm{d} \mathbf{x}}{\int_{B_{i}(t)}\left\|\mathbf{x}-\mathbf{x}_{i}(t)\right\|^{2} \mathrm{~d} \mathbf{x}} .
$$

In the same way, with $H_{0, \text { sym. }}^{1}(\Omega)=\left\{\boldsymbol{\sigma} \in H_{0}^{1}(\Omega), \boldsymbol{\sigma}=\boldsymbol{\sigma}^{\mathrm{t}}\right\}$, we now define the functional space related to the elastic stress tensor in the diffusive case $(\delta>0)$ :

$$
W_{B}:=\left\{\boldsymbol{\sigma} \in H_{0, \text { sym. }}^{1}(\Omega), \boldsymbol{\sigma}=0 \text { in } B\right\} .
$$

In the non-diffusive case $(\delta=0)$, denoting $L_{0, \text { sym. }}^{2}(\Omega)=\left\{\boldsymbol{\sigma} \in L^{2}(\Omega), \boldsymbol{\sigma}=\boldsymbol{\sigma}^{\mathrm{t}}\right\}$, we will rather consider

$$
W_{B}:=\left\{\boldsymbol{\sigma} \in L_{\text {sym. }}^{2}(\Omega), \boldsymbol{\sigma}=0 \text { in } B\right\} .
$$

The definition of $W_{B}$ consists in extending the elastic tensor by $\mathbf{0}$ in the rigid domain. Since rigid motions are incompressible, it is also convenient to extend the pressure field by 0 in the rigid domain:

$$
Z_{B}=\left\{p \in L_{0}^{2}(\Omega), p=0 \text { in } B\right\}
$$

where $L_{0}^{2}(\Omega)$ denotes the set of square-integrable functions with zero mean value. Hence the total Cauchy stress tensor $\boldsymbol{S}$, which is defined as $\boldsymbol{\Sigma}-p \mathbb{I}$, has been naturally extended by 0 in the rigid domain. At each time $t$, any function (u, $\boldsymbol{\sigma}, p$ ) satisfying the set of equations defined on $\Omega \backslash B(t)$ is extended on each $B_{i}(t)$ by

$$
\mathbf{u}(t, \mathbf{x})=\mathbf{v}_{i}(t)+\omega_{i}(t)\left(\mathbf{x}-\mathbf{x}_{i}\right)^{\perp}, \quad \boldsymbol{\sigma}(t, \mathbf{x})=0, \quad p(t, \mathbf{x})=0 .
$$

Note that $B$ evolves in time and so do the functional spaces. 


\section{Derivation of a variational formulation in the continuous framework}

Let us fix $t>0$, let $(\mathbf{u}, \boldsymbol{\sigma}, p)$ be an extended solution of the problem over the whole domain and choose $(\mathbf{w}, \boldsymbol{\tau}, q) \in$ $V_{B(t)} \times W_{B(t)} \times Z_{B(t)}$.

Step 1. Conservation of momentum. Denoting $D_{t}:=\partial_{t}+\mathbf{u} \cdot \nabla$ the total derivative operator (associated to the velocity field $\mathbf{u}$ ), we multiply Eq. (1) by $\mathbf{w}$ and integrate over the fluid domain $\Omega \backslash \overline{B(t)}$ :

$$
\int_{\Omega \backslash \overline{B(t)}} \rho_{\mathrm{f}} \mathrm{D}_{t} \mathbf{u}(t, \cdot) \cdot \mathbf{w}-\int_{\Omega \backslash \overline{B(t)}} \operatorname{div}(\mathbf{\Sigma}(t, \cdot)) \cdot \mathbf{w}+\int_{\Omega \backslash \overline{B(t)}} \nabla p(t, \cdot) \cdot \mathbf{w}=\int_{\Omega \backslash \overline{B(t)}} \mathbf{f}_{\mathrm{f}}(t, \cdot) \cdot \mathbf{w} \cdot
$$

Integrating by part and using Eq. (3) (and also Eq. (2)), we get:

$$
\begin{array}{r}
\int_{\Omega \backslash \overline{B(t)}} \rho_{\mathrm{f}} \mathrm{D}_{t} \mathbf{u}(t, \cdot) \cdot \mathbf{w}+2 \mu(1-r) \int_{\Omega \backslash \overline{B(t)}} \mathbb{D}(\mathbf{u}(t, \cdot)): \mathbb{D}(\mathbf{w})+\int_{\Omega \backslash \overline{B(t)}} \boldsymbol{\sigma}(t, \cdot): \mathbb{D}(\mathbf{w}) \\
-\int_{\Omega \backslash \overline{B(t)}} p(t, \cdot) \operatorname{div}(\mathbf{w})-\int_{\partial(\Omega \backslash \overline{B(t)})} \boldsymbol{S}(t, \cdot) \mathbf{n} \cdot \mathbf{w}=\int_{\Omega \backslash \overline{B(t)}} \mathbf{f}_{\mathrm{f}}(t, \cdot) \cdot \mathbf{w} .
\end{array}
$$

Some integrals over $\Omega \backslash \overline{B(t)}$ can be extended over $\Omega$ using the fact that, on the rigid domain $B(t), \mathbb{D}(\mathbf{w})=0, \operatorname{div}(\mathbf{w})=0$. Moreover, since $\mathbf{w} \in V_{B(t)}$, w takes the following form:

$$
\forall i, \quad \mathbf{w}(\mathbf{x})=\mathrm{T}_{i}[\mathbf{w}]+\mathrm{R}_{i}[\mathbf{w}]\left(\mathbf{x}-\mathbf{x}_{i}\right)^{\perp}, \text { in } B_{i}(t) .
$$

Taking advantage of the homogeneous boundary conditions on $\mathbf{u}$ at the boundary $\partial \Omega$ and the fluid-structure interaction at the interface described by Eqs. (10) and (11), we get

$$
\begin{array}{r}
\int_{\Omega \backslash \overline{B(t)}} \rho_{\mathrm{f}} \mathrm{D}_{t} \mathbf{u}(t, \cdot) \cdot \mathbf{w}+\sum_{i=1}^{N} m_{i} \dot{\mathrm{T}}_{i}[\mathbf{u}(t, \cdot)] \mathrm{T}_{i}[\mathbf{w}]+\sum_{i=1}^{N} J_{i} \dot{\mathrm{R}}_{i}[\mathbf{u}(t, \cdot)] \mathrm{R}_{i}[\mathbf{w}] \\
+2 \mu(1-r) \int_{\Omega} \mathbb{D}(\mathbf{u}(t, \cdot)): \mathbb{D}(\mathbf{w})+\int_{\Omega} \boldsymbol{\sigma}(t, \cdot): \mathbb{D}(\mathbf{w}) \\
-\int_{\Omega} p(t, \cdot) \operatorname{div}(\mathbf{w})=\int_{\Omega} \mathbf{f}(t, \cdot) \cdot \mathbf{w},
\end{array}
$$

with $\mathbf{f}(t, \cdot):=\mathbf{f}_{\mathrm{f}} \mathbf{1}_{\Omega \backslash \overline{B(t)}}+\sum_{i=1}^{N} \mathbf{f}_{i} \mathbf{1}_{B_{i}(t)}$. Finally, since we have

$$
\forall i, \quad m_{i} \dot{\mathrm{T}}_{i}[\mathbf{u}(t, \cdot)] \mathrm{T}_{i}[\mathbf{w}]+J_{i} \dot{\mathrm{R}}_{i}[\mathbf{u}(t, \cdot)] \mathrm{R}_{i}[\mathbf{w}]=\int_{B_{i}(t)} \rho_{i} \mathrm{D}_{t} \mathbf{u}(t, \cdot) \cdot \mathbf{w}
$$

we obtain the following equality:

$$
\begin{array}{r}
\int_{\Omega} \rho \mathrm{D}_{t} \mathbf{u}(t, \cdot) \cdot \mathbf{w}+2 \mu(1-r) \int_{\Omega} \mathbb{D}(\mathbf{u}(t, \cdot)): \mathbb{D}(\mathbf{w})+\int_{\Omega} \boldsymbol{\sigma}(t, \cdot): \mathbb{D}(\mathbf{w}) \\
-\int_{\Omega} p(t, \cdot) \operatorname{div}(\mathbf{w})=\int_{\Omega} \mathbf{f}(t, \cdot) \cdot \mathbf{w}
\end{array}
$$

with $\rho(t, \cdot):=\rho_{\mathrm{f}} \mathbf{1}_{\Omega \backslash \overline{B(t)}}+\sum_{i=1}^{N} \rho_{i} \mathbf{1}_{B_{i}(t)}$.

Step 2. Continuity equation. We multiply Eq. (2) by $q$ and integrate over the fluid domain $\Omega \backslash \overline{B(t)}$ :

$$
\int_{\Omega \backslash \overline{B(t)}} q \operatorname{div}(\mathbf{u}(t, \cdot))=0
$$

Since $q$ belongs to $Z_{B}$, it is equal to 0 in $B$. Hence the integral over the fluid domain can be extended over the whole domain:

$$
\int_{\Omega} q \operatorname{div}(\mathbf{u}(t, \cdot))=0 .
$$


Step 3. Oldroyd law. We multiply Eq. (4) by $\boldsymbol{\tau}$ and integrate over the fluid domain $\Omega \backslash \overline{B(t)}$ :

$$
\int_{\Omega \backslash \overline{B(t)}} \lambda\left(\mathrm{D}_{t} \boldsymbol{\sigma}(t, \cdot)+g_{a}(\nabla \mathbf{u}(t, \cdot), \boldsymbol{\sigma}(t, \cdot))\right): \boldsymbol{\tau}+\int_{\Omega \backslash \overline{B(t)}} \boldsymbol{\sigma}(t, \cdot): \boldsymbol{\tau}+\delta \int_{\Omega \backslash \overline{B(t)}} \nabla \boldsymbol{\sigma}(t, \cdot): \nabla \boldsymbol{\tau}=\int_{\Omega \backslash \overline{B(t)}} 2 r \mu \mathbb{D}(\mathbf{u}(t, \cdot)): \boldsymbol{\tau} .
$$

Since $\boldsymbol{\tau}$ belongs to $W_{B}$, it is equal to 0 in $B(t)$ and the integrals over the fluid domain can be extended over the whole domain:

$$
\int_{\Omega} \lambda\left(\mathrm{D}_{t} \boldsymbol{\sigma}(t, \cdot)+g_{a}(\nabla \mathbf{u}(t, \cdot), \boldsymbol{\sigma}(t, \cdot))\right): \boldsymbol{\tau}+\int_{\Omega} \boldsymbol{\sigma}(t, \cdot): \boldsymbol{\tau}+\delta \int_{\Omega} \nabla \boldsymbol{\sigma}(t, \cdot): \nabla \boldsymbol{\tau}=\int_{\Omega} 2 r \mu \mathbb{D}(\mathbf{u}(t, \cdot)): \boldsymbol{\tau} .
$$

Since the velocity is divergence free, $\rho$ is constant along the characteristics, i. e. $\mathrm{D}_{t} \rho=0$, and we have $\rho \mathrm{D}_{t} \mathbf{u}=\mathrm{D}_{t}(\rho \mathbf{u})$. Gathering the previous equalities, the fluid-structure interaction problem can be tackled with the following variational formulation (in which the time variable is omitted for the sake of simplicity):

$$
\left\{\begin{array}{l}
\text { Find }(\mathbf{u}(t, \cdot), \boldsymbol{\sigma}(t, \cdot), p(t, \cdot)) \in V_{B(t)} \times W_{B(t)} \times Z_{B(t)} \text { such that, for all }(\mathbf{w}, \boldsymbol{\tau}, q) \in V_{B(t)} \times W_{B(t)} \times Z_{B(t)}: \\
\bullet \int_{\Omega} \mathrm{D}_{t}(\rho \mathbf{u}) \cdot \mathbf{w}+2 \mu(1-r) \int_{\Omega} \mathbb{D}(\mathbf{u}): \mathbb{D}(\mathbf{w})+\int_{\Omega} \boldsymbol{\sigma}: \mathbb{D}(\mathbf{w})-\int_{\Omega} p \operatorname{div}(\mathbf{w})=\int_{\Omega} \mathbf{f} \cdot \mathbf{w}, \\
\bullet \int_{\Omega} q \operatorname{div}(\mathbf{u})=0 \\
\bullet \int_{\Omega} \lambda\left(\mathrm{D}_{t} \boldsymbol{\sigma}+g_{a}(\nabla \mathbf{u}, \boldsymbol{\sigma})\right): \boldsymbol{\tau}+\int_{\Omega} \boldsymbol{\sigma}: \boldsymbol{\tau}+\delta \int_{\Omega} \nabla \boldsymbol{\sigma}: \nabla \boldsymbol{\tau}=\int_{\Omega} 2 r \mu \mathbb{D}(\mathbf{u}): \boldsymbol{\tau} .
\end{array}\right.
$$

with the following generalized source terms and mass densities:

$$
\mathbf{f}(t, \cdot):=\mathbf{f}_{\mathbf{f}} \mathbf{1}_{\Omega \backslash \overline{B(t)}}+\sum_{i=1}^{N} \mathbf{f}_{i} \mathbf{1}_{B_{i}(t)}, \quad \rho(t, \cdot):=\rho_{\mathrm{f}} \mathbf{1}_{\Omega \backslash \overline{B(t)}}+\sum_{i=1}^{N} \rho_{i} \mathbf{1}_{B_{i}(t)} .
$$

\section{Algorithm}

\subsection{Semi-discretized formulation}

\section{Discretization of the total derivative operator}

Let us proceed to the time discretization. With classical notations, $f^{n}$ stands for the approximation of $f$ at time $t^{n}$. The time discretization is built upon the characteristics method which allows us to approximate the total derivative. Let us introduce the ODE

$$
\left\{\begin{array}{l}
\dot{\mathbf{X}}(s)=\mathbf{u}(s, \mathbf{X}(s)), \quad t \in\left(t^{n}, t^{n+1}\right) \\
\mathbf{X}\left(t^{n+1}\right)=\mathbf{x}
\end{array}\right.
$$

The solution of this ODE is denoted $s \mapsto \kappa\left(\mathbf{x}, t^{n+1} ; s\right)$. For any function $(t, \mathbf{x}) \mapsto f(t, \mathbf{x})$, we have

$$
\mathrm{D}_{t} f(t, \mathbf{x})=\left(\partial_{t} f+\mathbf{u} \cdot \nabla f\right)(t, \mathbf{x})=\left(\frac{\delta}{\delta s}(f(s, \kappa(\mathbf{x}, t ; s)))\right)_{\mid s=t} .
$$

Therefore, using the fact that $\kappa\left(\mathbf{x}, t^{n+1} ; t^{n+1}\right)=\mathbf{x}$, we can discretize the total derivative of $f$ as:

$$
\mathrm{D}_{t} f\left(t^{n+1}, \mathbf{x}\right) \simeq \frac{f^{n+1}(\mathbf{x})-f^{n} \circ \kappa^{n}(\mathbf{x})}{\Delta t}
$$

where $\kappa^{n}(\mathbf{x})$ is an approximation of $\kappa\left(\mathbf{x}, t^{n+1} ; t^{n}\right)$ which denotes the position at time $t^{n}$ of the point placed in $\mathbf{x}$ at time $t^{n+1}$ and moving along the integral path defined by the velocity field $\mathbf{u}$. Thus we will use in particular the approximations:

$$
\mathrm{D}_{t}(\rho \mathbf{u})\left(t^{n+1}, \cdot\right) \simeq \frac{\left(\rho^{n+1} \mathbf{u}^{n+1}\right)-\left(\rho^{n} \mathbf{u}^{n}\right) \circ \kappa^{n}}{\Delta t}, \quad \mathrm{D}_{t} \boldsymbol{\sigma}\left(t^{n+1}, \cdot\right) \simeq \frac{\boldsymbol{\sigma}^{n+1}-\boldsymbol{\sigma}^{n} \circ \kappa^{n}}{\Delta t} .
$$




\section{Splitting scheme}

Once the convective terms have been discretized using the characteristics method, we have to deal with the numerical treatment of the coupling between the momentum equation and the Oldroyd law. In order to simplify the computation, it is possible to relax the strong coupling by treating the term involving $\sigma$ in the momentum equation, i.e.

$$
\int_{\Omega} \boldsymbol{\sigma}(t, \cdot): \mathbb{D}(\mathbf{w})
$$

in an explicit way. By applying this splitting strategy, both equations become uncoupled at each time-step and can be solved independently. Such a formulation is often called "weakly coupled". The other possibility is of course to preserve the strong coupling at the discrete level, and thus treat this term implicitly. We will discuss further the advantage of such choices.

The algorithm reads as follows. Let $\left(\mathbf{u}^{n}, \boldsymbol{\sigma}^{n}\right)$ and $B^{n}$ be given.

1. Compute the updated position of the particles $\left(\mathbf{x}_{i}^{n+1}, \theta_{i}^{n+1}\right)$, hence defining the rigid domain $B^{n+1}=B\left(t^{n+1}\right)$ : computing $\mathbf{v}_{i}^{n}=\mathrm{T}_{i}\left[\mathbf{u}^{n}\right]$ and $\omega_{i}^{n}=\mathrm{R}_{i}\left[\mathbf{u}^{n}\right]$, we define

$$
\mathbf{x}_{i}^{n+1}=\mathbf{x}_{i}^{n}+\Delta t \mathbf{v}_{i}^{n}, \quad \theta_{i}^{n+1}=\theta_{i}^{n}+\Delta t \omega_{i}^{n} ;
$$

The definition of $\left(\mathbf{x}_{i}^{n+1}, \theta_{i}^{n+1}\right)$ also defines $B^{n+1}$ by using the geometrical characteristics of the particle ;

2. Compute the source terme $\mathbf{f}^{n+1}$ and the mass density $\rho^{n+1}$ :

$$
\mathbf{f}^{n+1}=\mathbf{f}_{\mathrm{f}} \mathbf{1}_{\Omega \backslash \overline{B^{n+1}}}+\sum_{i=1}^{N} \mathbf{f}_{i} \mathbf{1}_{B_{i}^{n+1}}, \quad \rho^{n+1}=\rho_{\mathrm{f}} \mathbf{1}_{\Omega \backslash \overline{B^{n+1}}}+\sum_{i=1}^{N} \rho_{i} \mathbf{1}_{B_{i}^{n+1}}
$$

3. Solve the strongly coupled variational problem

$$
\left(\mathrm{P}_{\text {impl. }}^{n+1}\right)\left\{\begin{array}{r}
\text { Find }\left(\mathbf{u}^{n+1}, p^{n+1}\right) \in V_{B^{n+1}} \times Z_{B^{n+1}} \text { such that, for all }(\mathbf{w}, q) \in V_{B^{n+1}} \times Z_{B^{n+1}}: \\
\frac{1}{\Delta t} \int_{\Omega} \rho^{n+1} \mathbf{u}^{n+1} \cdot \mathbf{w}+2 \mu(1-r) \int_{\Omega} \mathbb{D}\left(\mathbf{u}^{n+1}\right): \mathbb{D}(\mathbf{w})-\int_{\Omega} p^{n+1} \operatorname{div}(\mathbf{w})+\int_{\Omega} q \operatorname{div}\left(\mathbf{u}^{n+1}\right) \\
\quad=\frac{1}{\Delta t} \int_{\Omega}\left(\left(\rho^{n} \mathbf{u}^{n}\right) \circ \kappa^{n}\right) \cdot \mathbf{w}-\int_{\Omega} \boldsymbol{\sigma}^{n+1}: \mathbb{D}(\mathbf{w})+\int_{\Omega} \mathbf{f}^{n+1} \cdot \mathbf{w} . \\
\text { Find } \boldsymbol{\sigma}^{n+1} \in W_{B^{n+1}} \text { such that, forall } \boldsymbol{\tau} \in W_{B^{n+1}}: \\
\frac{\lambda}{\Delta t} \int_{\Omega} \boldsymbol{\sigma}^{n+1}: \boldsymbol{\tau}+\lambda \int_{\Omega} g_{a}\left(\nabla \mathbf{u}^{n+1}, \boldsymbol{\sigma}^{n+1}\right): \boldsymbol{\tau}+\int_{\Omega} \boldsymbol{\sigma}^{n+1}: \boldsymbol{\tau}+\delta \int_{\Omega} \nabla \boldsymbol{\sigma}^{n+1}: \nabla \boldsymbol{\tau} \\
=\frac{\lambda}{\Delta t} \int_{\Omega}\left(\boldsymbol{\sigma}^{n} \circ \kappa^{n}\right): \boldsymbol{\tau}+\int_{\Omega} 2 r \mu \mathbb{D}\left(\mathbf{u}^{n+1}\right): \boldsymbol{\tau} .
\end{array}\right.
$$

or, alternatively, the weakly coupled problem

$$
\left(\mathrm{P}_{\text {expl. }}^{n+1}\right)\left\{\begin{array}{c}
\text { Find }\left(\mathbf{u}^{n+1}, p^{n+1}\right) \in V_{B^{n+1}} \times Z_{B^{n+1}} \text { such that, for all }(\mathbf{w}, q) \in V_{B^{n+1}} \times Z_{B^{n+1}}: \\
\frac{1}{\Delta t} \int_{\Omega} \rho^{n+1} \mathbf{u}^{n+1} \cdot \mathbf{w}+2 \mu(1-r) \int_{\Omega} \mathbb{D}\left(\mathbf{u}^{n+1}\right): \mathbb{D}(\mathbf{w})-\int_{\Omega} p^{n+1} \operatorname{div}(\mathbf{w})+\int_{\Omega} q \operatorname{div}\left(\mathbf{u}^{n+1}\right) \\
\quad=\frac{1}{\Delta t} \int_{\Omega}\left(\left(\rho^{n} \mathbf{u}^{n}\right) \circ \kappa^{n}\right) \cdot \mathbf{w}-\int_{\Omega} \sigma^{n}: \mathbb{D}(\mathbf{w})+\int_{\Omega} \mathbf{f}^{n+1} \cdot \mathbf{w} . \\
\text { Find } \boldsymbol{\sigma}^{n+1} \in W_{B^{n+1}} \text { such that }, \text { forall } \boldsymbol{\tau} \in W_{B^{n+1}}: \\
\frac{\lambda}{\Delta t} \int_{\Omega} \boldsymbol{\sigma}^{n+1}: \boldsymbol{\tau}+\lambda \int_{\Omega} g_{a}\left(\nabla \mathbf{u}^{n+1}, \boldsymbol{\sigma}^{n+1}\right): \boldsymbol{\tau}+\int_{\Omega} \boldsymbol{\sigma}^{n+1}: \boldsymbol{\tau}+\delta \int_{\Omega} \nabla \boldsymbol{\sigma}^{n+1}: \nabla \boldsymbol{\tau} \\
=\frac{\lambda}{\Delta t} \int_{\Omega}\left(\boldsymbol{\sigma}^{n} \circ \kappa^{n}\right): \boldsymbol{\tau}+\int_{\Omega} 2 r \mu \mathbb{D}\left(\mathbf{u}^{n+1}\right): \boldsymbol{\tau} .
\end{array}\right.
$$

Problems $\left(\mathrm{P}_{\text {impl. }}^{n+1}\right)$ and $\left(\mathrm{P}_{\text {expl. }}^{n+1}\right)$ are both consistent with the continuous equations. However, several differences and links between the two formulations can be described: 
- The variational formulations $\left(\mathrm{P}_{\text {impl. }}^{n+1}\right)$ and $\left(\mathrm{P}_{\text {expl. }}^{n+1}\right)$ both take advantage of the characteristic method: the nonlinearity associated to the total derivative of the velocity field (in the momentum equation) and the elastic tensor (in the Oldroyd equation) is tackled numerically by the characteristics method which is based upon the identification of $\mathbf{x} \mapsto \kappa^{n}(\mathbf{x})$ which approximates the position at time $t^{n}$ of the point placed in $\mathbf{x}$ at time $t^{n+1}$ and moving along the integral path defined by the velocity field $\mathbf{u}$. In practice, the foot of the characteristic curve is approximated by using the velocity field $\mathbf{u}^{n}$.

- Several properties emerge from straightforward adaptations of [11: first, at each time step, the nonlinear problem $\left(\mathrm{P}_{\mathrm{impl}}^{n+1}\right)$ admits a unique solution for sufficiently small data. Second, in the corotational case, namely for $a=0$, we can take advantage of the particular structure of the Oldroyd system: existence of a solution at each time step is guaranteed without smallness assumption on the data and, besides, the numerical stability of the discretized-in-time solution can be derived by using classical energy estimates. This motivates the restriction of the numerical results in this paper to the corotational case, which is the only one for which existence of a solution with no restriction on the data, as well as stability of the numerical approximation have been proved.

- The weakly coupled scheme $\left(\mathrm{P}_{\text {expl. }}^{n+1}\right)$ relies on the fact that the term

$$
\int_{\Omega} \boldsymbol{\sigma}(t, \cdot): \mathbb{D}(\mathbf{w})
$$

in the momentum equation is treated explicitly, i.e. it is evaluated at time $t^{n}$ instead of $t^{n+1}$. This explicit treatment not only allows us to uncouple the momentum equation and the Oldroyd law at each time-step but it also leads to a linearization of the Oldroyd law. In fact, it leads to the numerical resolution of two linear problems: the first one is a generalized linear Stokes system (including a momentum equation associated to the continuity equation) and the second one is a linear advection-reaction system.

- A fixed-point procedure based upon solving iteratively $\left(\mathrm{P}_{\text {expl. }}^{n+1}\right)$ with an update of the explicit term leads to the determination of the solution of $\left(\mathrm{P}_{\mathrm{impl}}^{n+1}\right)$. However, numerical computations have shown no qualitative difference between the splitting method (that consists in solving problem $\left(\mathrm{P}_{\text {expl. }}^{n+1}\right)$ only once at each time-step) and the fixedpoint method.

In practice, when targeting the simulation of the long-time behavior of dense suspensions, the formulation based on the splitting scheme will be used for faster computations. At variance with the implicit scheme, no stability analysis is available in this framework. However, all numerical tests performed have shown stable results. Still, the variational formulation $\left(\mathrm{P}_{\text {expl. }}^{n+1}\right)$ contains a major issue that needs to be addressed. From the numerical point of view, the main difficulty in computing the solution of the splitting scheme consists in solving successively two linear variational problems dealing with constrained functional spaces which are non-standard. First, the definition of a finite element basis for each functional space is rather delicate. Second, as the rigid domain evolves in times, so do the constraints defining the functional spaces, leading to a new definition of a finite element basis associated to the functional spaces. This difficulty is overcome by the penalty method which relaxes the constraints expressed in the functional spaces by adding a penalty term in the variational formulation. The penalty method leads to the possibility to implement the method with any standard finite element solver that is able to solve a Stokes-like problem (with e.g. $\mathbb{P}_{1}^{\mathrm{b}}-\mathbb{P}_{1}$ elements) and a linear advection-reaction(-diffusion) system (with e.g. $\mathbb{P}_{1}$ elements in the diffusive case, $\mathbb{P}_{0}$ elements in the non-diffusive case).

\subsection{Penalty method}

As already mentioned, the constraints expressed in the functional spaces have to be tackled from the numerical point of view. One possibility is to use an iterative algorithm on an auxiliary field, composed by Lagrange multipliers, which warrants the rigid motion constraint of the particles. An alternative to this iterative process are penalty methods: the rigid motion constraint is obtained by relaxing the definition of the functional spaces and adding penalty terms in the variational formulation. In the end, we obtain a mixed formulation which is well-posed and very easy to compute with a usual finite element solver.

In the case of the diffusive Oldroyd model $(\delta>0)$, the variational formulation $\left(\mathrm{P}_{\text {expl. }}^{n+1}\right)$ is thus approximated by the 
penalized one:

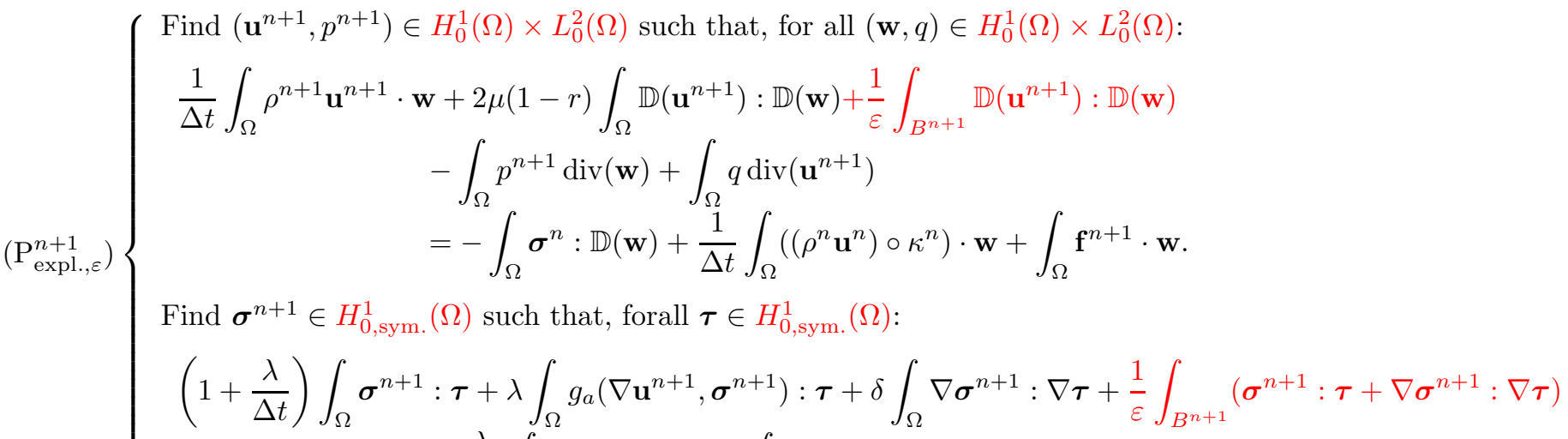

$$
\begin{aligned}
& =\frac{\lambda}{\Delta t} \int_{\Omega}\left(\boldsymbol{\sigma}^{n} \circ \kappa^{n}\right): \boldsymbol{\tau}+\int_{\Omega} 2 r \mu \mathbb{D}\left(\mathbf{u}^{n+1}\right): \boldsymbol{\tau} \text {. }
\end{aligned}
$$

Let us give some comments on the penalty method. The penalty term related to $\mathbb{D}\left(\mathbf{u}^{n+1}\right)$ consists in replacing rigid zones by highly viscous ones. In the same way the penalty term related to $\boldsymbol{\sigma}^{n+1}$ implies that the elastic tensor is (close to) 0 in the rigid domain, as expected. In the diffusive Oldroyd model $\delta>0$, the functional framework for the elastic stress tensor is $H^{1}$. As a consequence the $H^{1}$-penalization of the elastic stress tensor (i.e. with the term $\left.\frac{1}{\varepsilon} \int_{B^{n+1}}\left(\boldsymbol{\sigma}^{n+1}: \boldsymbol{\tau}+\nabla \boldsymbol{\sigma}^{n+1}: \nabla \boldsymbol{\tau}\right)\right)$ is more accurate than the classical $L^{2}$-penalization (with a term $\frac{1}{\varepsilon} \int_{B^{n+1}}\left(\boldsymbol{\sigma}^{n+1}: \boldsymbol{\tau}\right)$ ). In fact we can prove that the penalized velocity field converges to the exact velocity field at order 1 in the $H^{1}$-norm when $\varepsilon$ vanishes.

In the non-diffusive Oldroyd model, the functional framework for the elastic stress tensor is $L^{2}$. As a consequence, the $L^{2}$-penalization of the stress tensor in the constitutive equation ensures the fact that the penalized stress tensor converges to the exact stress tensor at order 1 in the $L^{2}$-norm. Besides, $\mathbb{P}_{0}$ finite elements for the elastic stress have to be preferred over $\mathbb{P}_{1}$ finite elements, since they do not require the continuity of the elastic stress tensor at the fluid-structure interface, and allow to reduce computational costs. Note that when using $\mathbb{P}_{0}$ finite elements, the gradient of the solution is zero on each mesh element. Nevertheless, since we use characteristics method for the transport term, the discretized equations do not involve the computation of the gradient of the solution. The variational formulation $\left(\mathrm{P}_{\text {expl. }}^{n+1}\right)$ is in this case approximated by the following penalized one:

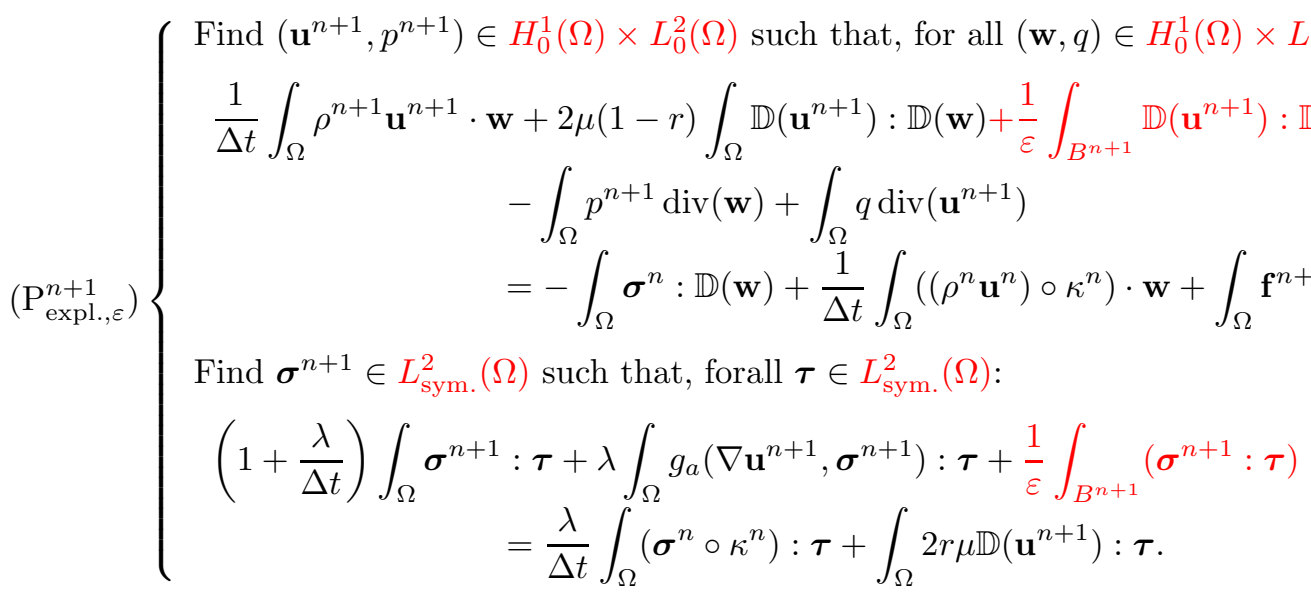

Let us give some comprehensive details on the practical computations. The convergence of numerical methods is ensured by classical analysis results. For instance, under regularity assumptions on the solution, the use of $P_{\mathrm{b}}^{1} / P^{1}$ finite elements for the Stokes-type equation ensures convergence in space of the velocity field at order 1 in the $H^{1}-$ norm. The stress also converges in space at order 1 in the $L^{2}$ norm with $P^{0}$ elements in the standard case, in the $H^{1}$ norm with $P^{1}$ elements in the diffusive case. Besides the characteristics method ensures convergence in time at order 1 . For the computational aspects, when considering the penalized Stokes-type problem of $\left(\mathrm{P}_{\text {expl., },}^{n+1}\right)$, the profile of the matrix resulting 
from the finite element discretization exactly matches the profile of the matrix without penalty term. This statement holds also for the penalized Oldroyd equation of problem $\left(\mathrm{P}_{\text {expl., }}^{n+1}\right)$ with respect to its unpenalized version. Nevertheless, the penalty method suffers from the following drawback: the conditioning of the matrix tends to deteriorate. Hence at each time step, the solver associated to the linear problem should be checked carefully: in particular, iterative solvers may fail to compute the numerical solution (although the penalized problem is well-posed) because of the bad conditioning. Without pre-conditioning techniques, direct solvers have to be used and, as a practical consequence, this leads to a limitation of the method to 2D flows: indeed 3D flows are associated to a larger number of degrees of freedom which prevents us from performing the resolution of the linear problems with a direct solver.

Let us discuss the (near-)contact issue in the practical computations. Assuming, as we have in this model, that particle surfaces are perfectly smooth and that Stokes-like models are valid at any scale, it is known that contacts are not supposed to happen (no contact in finite time) 24, 26, 27. In practice, because of time discretization and inexact estimate of lubrication forces, the "do-nothing" approach is risky, as contact or overlapping between rigid entities may happen. In order to avoid overlapping, refinement of the time step may help but, in the case of dense suspensions, the quasicontact situation would lead to severe limitations of the time step which would drastically limit the advance-in-time of the simulation. Therefore a robust handling of the contact is needed in order to avoid overlapping without imposing a severe restriction on the time step. The treatment of possible overlaps is even more important in the case of dense suspensions, if we want to avoid small time steps. For that purpose we use a projection algorithm, where inelastic collisions between rigid particles are computed. It consists of projecting the velocity field onto some convex admissible set depending on the current configuration, so that particles do not overlap, see 44, 39. A finer description of the near-contact dynamics could be carried out by integrating lubrication models as in [40, 25.

\section{$5 \quad$ Numerical simulations}

In the simulations, the domain is $\left(-\frac{\mathrm{L}_{x}}{2},+\frac{\mathrm{L}_{x}}{2}\right) \times\left(-\frac{\mathrm{L}_{y}}{2},+\frac{\mathrm{L}_{y}}{2}\right)$. The viscoelastic fluid is characterized by the viscosity $\mu$, the retardation parameter $r$, the density $\rho_{\mathrm{f}}$ and the relaxation time $\lambda$. For the reasons presented in the previous section, we only consider the corotational case, namely $a=0$. In fact, the other cases present stability issues that are independent of the coupling with rigid particles. The $N$ particles are defined by their semi-axes $R_{1}$ and $R_{2}$ (with $R_{1} / R_{2}=1$ for a disk) and density $\rho_{B}$. Gravity constant is denoted $g$. Numerical parameters related to the discretization (in time and in space) are denoted by $\Delta t$ and $h$. Data related to each simulation can be found in Tables 1 and 2, The penalty parameter $\varepsilon$ involved in the rigidity constraint is set to $10^{-8}$ in all the simulations.

\subsection{Sedimentation of circular particles}

Sedimentation of spherical particles is investigated in this subsection. We define for that purpose the Reynolds, Weissenberg and elasticity numbers:

$$
\mathrm{Re}=\frac{\rho_{\mathrm{f}} U R}{\mu}, \quad \mathrm{We}=\frac{U \lambda}{R}, \quad \mathrm{E}=\frac{\mathrm{We}}{\mathrm{Re}} .
$$

where $U$ scales the magnitude of the velocity, $R$ is the radius of the particle (other quantities have been already introduced).

\subsubsection{Single particle}

Sedimentation of a single circular particle in the non-inertial regime is described in Figures 34 Inertial terms are neglected and we therefore consider the Stokes-Oldroyd equations for the fluid. Data are given in Table $10\left[\mathrm{~A}_{1}\right]$ : the governing non-dimensional numbers are therefore $\mathrm{Re}=0, \mathrm{We}=64$ and $\mathrm{E}=+\infty$. Buoyancy is fixed and equal to $\Delta \rho=\rho_{B}-\rho_{\mathrm{f}}=1$.

- Figure 3 presents the sedimentation of the disk in a viscoelastic fluid at different time steps, highlighting the evolution of the sedimenting velocity from the initial position to the near-contact position.

- Figure 4 present the evolution of the shear contribution and the elongational contribution of the elastic tensor: in particular, once the disk reaches the bottom of the domain, the fluid velocity is nearly homogeneous and the relaxation of the elastic characteristics towards the homogeneous equilibrium distribution is observed with an exponential decay associated to the characteristic relaxation time $\lambda$. 
Then we add inertial effects and investigate the influence of the Reynolds number and model parameters on the sedimentation of the particle. Data are given in Table $1\left[\mathrm{~A}_{2}\right]$ and all results are compared to the Newtonian case with constant viscosity $(1-r) \mu$.

- Figure[5allows us to discuss the influence of the retardation parameter over the sedimentation in non-inertial regimes and inertial regimes. In the experiment, the Reynolds number varies from 0 to 60 , whereas the Weissenberg number is about 80 (hence the elasticity number varies from 1 to $+\infty$. Numerical results tend to show that the sedimenting velocity for this value of the Weissenberg number (hence for a large value of $\lambda$ ) is close to the one obtained with a Newtonian fluid with viscosity $(1-r) \mu$.

- Figure 6 allows us to discuss the influence of the elastic relaxation over the sedimentation in non-inertial regimes and inertial regimes. In the experiment, the Reynolds number varies from 0 to 60 , whereas the Weissenberg number varies between 0 and 8: in each subfigure, the Reynolds number is fixed ( $R e=0,12.5,25$ and 60 respectively), hence leading to elasticity numbers that range from 0 to $+\infty$. When focusing on moderate values of $\lambda$, the simulation shows a sedimentation dynamics that qualitatively lies between the Newtonian cases with viscosities $\mu$ and $(1-r) \mu$.

These results show that increasing the elastic properties of the bulk fluid decreases the sedimenting velocity: viscoelastic fluids modeled by the Oldroyd law are shear-thinning. However inertial effects tend to damp the differences due to the elasticity of the fluid, as it enhances the mixing of the fluid.

As a concluding remark, we illustrate the influence of the mesh size and time step of the numerical method for the particle motion in the case of the sedimentation of a sphere in a viscoelastic fluid, including inertial effects. Note that no data in the literature afford the validation of numerical methods for the fluid-structure problem with a viscoelastic fluid; however the axisymmetric computation of a sphere falling in a vertical tube was tested in [16] and, for validation purposes, compared with computations related to a circular cylinder fixed at different eccentric positions in a creeping channel flow that is steady and non-inertial. Our convergence study is based upon the data related to Expriment $\left[\mathrm{A}_{2}\right]$ : the domain is $[-1,1] \times[-3,3]$, the fluid is viscoelastic $\left(\rho_{\mathrm{f}}=0.1, \mu=0.01, r=0.5, \lambda=0.1, \delta=0, a=0\right)$, the particle is a sphere $\left(R_{1}=R_{2}=0.125\right)$ with density $\rho_{B}=1+\rho_{\mathrm{f}}$ and initial position $(0,2.5)$. In Figure 7, the mesh size is fixed to $h=1 / 50$ and we let $\Delta t$ vary between 0.030 (coarse time step) and 0.005. The evolution of the position of the particle $t \mapsto(x(t), y(t))$ is computed for these sets of data. In Figure 8, the time step is fixed to $\Delta t=0.005$ and we let $h$ vary between $1 / 20$ (coarse mesh) and 1/100. In both cases, the convergence is illustrated through a stabilization process of the trajectory of the particle $t \mapsto y(t)$ (where $y(t)$ denotes the altitude of the particle at time $t$ ) for small values of $\Delta t$ and $h$. The method being stable and consistent (at least in the corotational case which is considered here), this ensures the accuracy of the method. Note that computations related to Expriments $\left[\mathrm{A}_{1}\right]$ and $\left[\mathrm{A}_{2}\right]$ have been made with $\Delta t=0.005$ and $h=1 / 50$ : these discretization parameters fall into the range of values leading to a numerical solution which is close to the reference solution.

\subsubsection{Pairwise interactions}

As pointed out in [17, the phenomenon of drafting and kissing and tumbling emerges from the conjunction of inertial effects associated with wakes. The interaction between two sedimenting disks is investigated in a rectangular domain $[-1,1] \times[-5,5]$. The initial positions of the disks are:

$$
\mathbf{x}^{(1)}(0)=\left(\begin{array}{c}
+0.000 \\
+2.000
\end{array}\right), \quad \mathbf{x}^{(2)}(0)=\left(\begin{array}{c}
-0.005 \\
+1.625
\end{array}\right)
$$

and other data are described in Table1, referred as experiment [B]. The phenomenon is captured in the Newtonian setting, see Figure 9, but it is highly sensitive to perturbation. Indeed, introducing some elasticity in the model, namely $r=0.5$ and $\lambda=0.10$, a similar numerical experiment in the viscoelastic case is reproduced in Figure 10, using the same initial conditions. The Newtonian case is obtained in the regime $[\mathrm{Re}, \mathrm{We}, \mathrm{E}]=[75,0,0]$ whereas the viscoelastic case corresponds to the regime $[\mathrm{Re}, \mathrm{We}, \mathrm{E}]=[150,20,0.12]$. We may observe that in the viscoelastic case the dynamics differ in terms of rearrangement mechanisms: one disk is captured in the wake of the other but the kissing phase is much longer than in the Newtonian case, resulting in the absence of tumbling phase. Figures 11 and 12 present the dynamics of the shear contribution and the elongational contribution of the elastic tensor, resulting from the sedimentation of the disks. As noticed in [37, if two touching disks are launched side-by-side in a Newtonian fluid, they will be pushed apart until a stable separation distance between centers across the stream is established. If the same two disks are launched from an 
initial side-by-side configuration, the disks will attract, turn and chain. This was referred as a dispersion phenomenon in the Newtonian liquid and aggregation phenomenon in the viscoelastic liquid. Numerical simulations presented in Figures 10 outline the influence of the aggregation phenomenon in the dynamics of the two sedimenting disks. Note that the chaining in the settling direction is observed in the experiment: the particles form a long body that is stable and does not tumble.

\subsubsection{Sedimentation in dense suspensions}

Data are described in Table 1, referred as experiment [C]. Sedimentation of the particles can be observed in Figure 13I. for the Newtonian case (corresponding to the regime [Re, We, E] $=[2,0,0]$ ), and Figures [13 II. and [14 for the viscoelastic case (corresponding to the regime $[\mathrm{Re}, \mathrm{We}, \mathrm{E}]=[2,12,6]$ ). Because of the inertial and congestion effects, it can be observed that the dynamics of sedimentation in the viscoelastic case does not differ a lot from the one that can be observed in the Newtonian case.

\subsection{Shear flows}

Shear flows including rigid ellipses are investigated in this subsection. We define for that purpose the Reynolds, Weissenberg and elasticity numbers as proposed in [15:

$$
\operatorname{Re}=\frac{\rho_{\mathrm{f}} G\left(2 R_{1}\right)^{2}}{\mu},
$$

where $R_{1}$ is the length of the semi-major axis of the ellipse, $G$ is the shear rate. Using the typical velocity $U=G\left(2 R_{1}\right)$, we define the Weissenberg and elasticty numbers as

$$
\mathrm{We}=G \lambda, \quad \mathrm{E}=\frac{\mathrm{We}}{\mathrm{Re}} .
$$

Newtonian fluids in the non-inertial regime. At low Reynolds numbers, when inertial effects are neglectable, the effective viscosity of a suspension of passive circular particles in a fluid of viscosity $\mu$ depends on its volume fraction $\phi$. In the dilute regime, where $\phi \ll 1$ so that particles do not interact, the effective viscosity is described in a suitable manner by Einstein's relation

$$
\mu_{\mathrm{eff} .} \simeq \mu(1+\alpha \phi),
$$

where $\alpha$ is known as Einstein's coefficient. It depends on the dimension and on the elongation of entities. In the case of circular and passive particles, $\alpha=(2+d) / 2$, where $d$ is the space dimension. In particular $\alpha=2$ in $2 d$ (see $\underline{3}$ ). The linear dependency with respect to volume fraction is due to the fact that, in this regime, the total effect of the particles on the viscosity is equal to the sum of individual contributions. Since the particles are circular, each contribution is identical (if we neglect those which are close to the plates). When particles are elongated, the contribution to instantaneous apparent viscosity due to each particle depends on its orientation, as expressed in 34 resulting in establishing that Einstein's relation (14) is still valid, but the constant $\alpha$ depends on the elongation ratio. Beyond the dilute regime, particles interact and thus simple addition of contributions is no more valid. In concentrated regimes, experimental results are fitted by the following semi-empirical law proposed by [38]:

$$
\mu_{\mathrm{eff} .}=\mu\left(1-\frac{\phi}{\phi_{m}}\right)^{-\alpha \phi_{m}}
$$

where $\phi_{m}$ define a maximal packing volume fraction.

Viscoelastic fluids. The rheology of viscoelastic fluids of Oldroyd type can be investigated numerically from dilute suspensions to dense suspensions, with circular or ellipsoidal particles. This approach can be analysed as a supplementary benchmark to address the classification of viscoelastic fluids as an Oldroyd-type fluid, by fitting numerical simulations to experiments.

Methodology. We consider a 2D rheometer $\left(-\frac{\mathrm{L}_{x}}{2},+\frac{\mathrm{L}_{x}}{2}\right) \times\left(-\frac{\mathrm{L}_{y}}{2},+\frac{\mathrm{L}_{y}}{2}\right)$, see Figure 1. The fluid adheres to the moving walls $y= \pm \frac{\mathrm{L}_{y}}{2}$ whereas periodic boundary conditions are considered at $x= \pm \frac{\mathrm{L}_{x}}{2}$. The Cauchy tensor is defined as $\boldsymbol{\Sigma}_{\text {tot. }}=p \mathrm{I}-\boldsymbol{\Sigma}$ with

$$
\boldsymbol{\Sigma}= \begin{cases}2 \mu \mathbb{D}(\mathbf{u}), & \text { for a Newtonian fluid } \\ 2 \mu(1-r) \mathbb{D}(\mathbf{u})+\boldsymbol{\sigma}, & \text { for a viscoelastic fluid }\end{cases}
$$


Tangential (shear) forces exerted by the walls over the fluid read

$$
\mathcal{F}_{\mid y= \pm \frac{L_{y}}{2}}=\int_{-\frac{L_{x}}{2}}^{+\frac{L_{x}}{2}}\left( \pm \mathbf{e}_{x}\right) \cdot\left(-\boldsymbol{\Sigma}_{\text {tot. }}\right)_{\mid y= \pm \frac{L_{y}}{2}} \cdot\left( \pm \mathbf{e}_{y}\right) \mathrm{d} x .
$$

Let us discuss the different cases:

- In the Newtonian case with no rigid inclusion, the Couette velocity profile is linear, see Figure 2.

$$
\mathbf{u}(x, y)=S \frac{y}{L_{y} / 2} \mathbf{e}_{x}, \quad p(x, y)=0
$$

and since we have $\Sigma=2 \mu \mathbb{D}(\mathbf{u})$, straightforward computations lead to the following relation:

$$
\mu=\frac{L_{y}}{4 L_{x} S}\left(\mathcal{F}_{\mid y=+\frac{L_{y}}{2}}+\mathcal{F}_{\mid y=-\frac{L_{y}}{2}}\right) .
$$

- In the viscoelastic case with no rigid inclusion, the effective viscosity is defined as:

$$
\mu_{\mathrm{eff} .}:=\frac{L_{y}}{4 L_{x} S}\left(\mathcal{F}_{\mid y=+\frac{L_{y}}{2}}+\mathcal{F}_{\mid y=-\frac{L_{y}}{2}}\right),
$$

in which the forces now depend on the viscoelastic tensor $\boldsymbol{\Sigma}=2 \mu(1-r) \mathbb{D}(\mathbf{u})+\boldsymbol{\sigma}$.

Dealing with rigid inclusions does not alter the definition of the effective viscosity: rigid particles do modify the velocity profiles, see Figure 2, but only the bulk fluid exerts forces on the walls. Thus the influence of the particles on the effective viscosity is taken into account through the possible modifications of the Couette profile which results in a modification of $\Sigma$ (with a Newtonian or viscoelastic model, according to the fluid) at the walls.

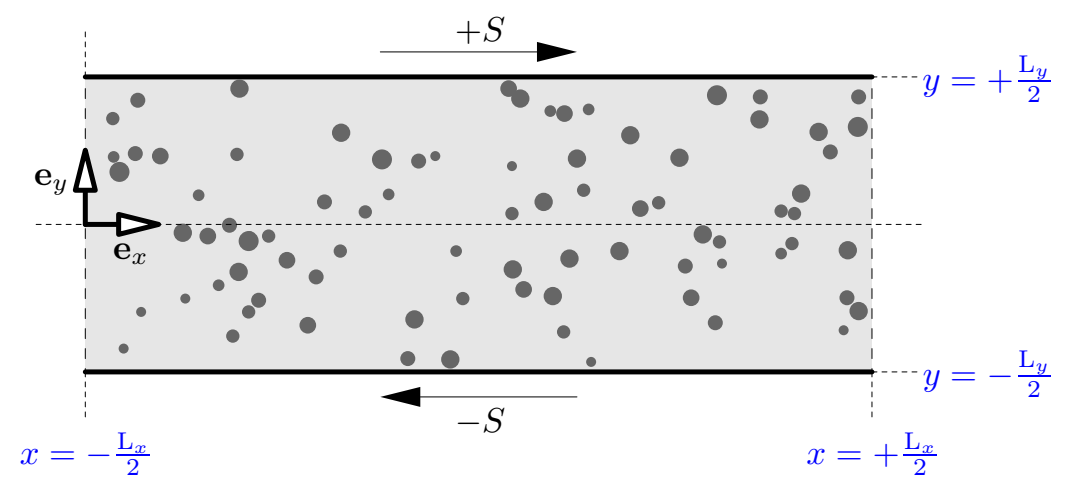

Figure 1: Dimensions of a two-dimensional rheometer.

a)

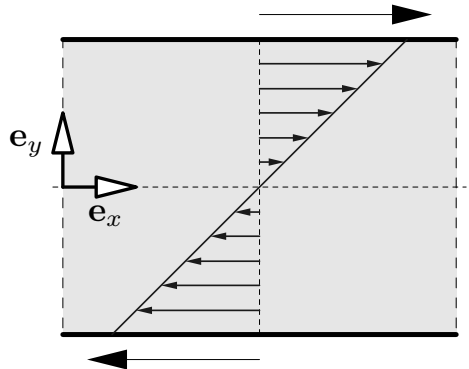

b)

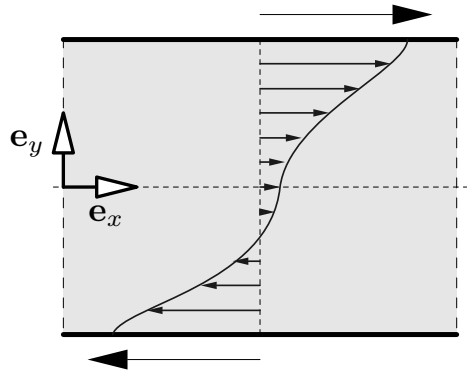

Figure 2: Velocity profile in the viscosimeter: a) in the Newtonian case; b) in a non-Newtonian case. 


\subsubsection{Preliminary study: numerical validation in the non-inertial regime}

Rheology of a Newtonian fluid with rigid inclusions in the dilute regime. In numerical simulations, referred as experiment [D] we address the computation of the effective viscosity of a suspension with circular entities in the Newtonian fluid in the non-inertial regime $(\mathrm{Re}=0)$ (with data provided in Table $2[\mathrm{D}])$. Numerical results, see Table 3 , are in good agreement with the theoretical results derived by Einstein: coefficient $\alpha$ in Eq. (14) is obtained in the dilute regime even if, as it is expected, the validity of the Einstein formula falls down as the solid fraction increases.

Rheology of a viscoelastic fluid with no rigid inclusion. The rheology of a viscoelastic fluid of Oldroyd type (with no inclusion) has been analyzed in different works, see in particular the review [50] and references therein. Considering the two-dimensional rheometer described in Figure 1, plane shear flow can be described by the velocity, pressure and extra-stress:

$$
\mathbf{u}(x, y)=(u(y), 0), \quad p(x, y)=0, \quad \boldsymbol{\sigma}(x, y)=\left(\begin{array}{ll}
\sigma_{x x} & \sigma_{x y} \\
\sigma_{x y} & \sigma_{y y}
\end{array}\right)
$$

with

$$
u(y)=G y, \quad \sigma_{x y}=\mu G \frac{r}{1+\mathrm{We}^{2}\left(1-a^{2}\right)}, \quad \sigma_{x x}=\mathrm{We}(1+a) \sigma_{x y}, \quad \sigma_{y y}=-\mathrm{We}(1-a) \sigma_{x y}
$$

where $G:=\frac{S}{L_{y} / 2}$ is the shear rate (in reciprocal seconds) and We $:=\lambda G$ denotes the (dimensionless) Weissenberg number. The total shear stress is

$$
\begin{aligned}
\Sigma_{x y} & =(1-r) \mu u^{\prime}+\sigma_{x y} \\
& =\mu G\left(1-r+\frac{r}{1+\mathrm{We}^{2}\left(1-a^{2}\right)}\right) .
\end{aligned}
$$

leading to the theoretical effective viscosity:

$$
\mu_{\text {eff.,th. }}=\mu\left(1-r+\frac{r}{1+\mathrm{We}^{2}\left(1-a^{2}\right)}\right) .
$$

We address the computation of the effective viscosity of a viscoelastic fluid in numerical simulations in the non-inertial regime, referred as experiment [E], see Table 2, Figure 15 presents the rheology of fluid with no rigid inclusion. First we may notice that the effective viscosity reaches a constant value after some time. Starting from a situation at rest, the stationary state is attained after a characteristic time which depends on both the elastic relaxation time and the shear rate. The constant value that is numerically captured fits the theoretical value of the effective viscosity, see Eq. (16), with an error rate which is less than $1 \%$ for all different values of the relaxation time $\lambda$. Second it is observed that increasing the value of the relaxation time tends to lower the effective viscosity. Fixing the relaxation time in the Weissenberg number, increasing the shear velocity produces a similar effect. As a consequence, viscoelastic fluids of the Oldroyd type, for $a \neq \pm 1$, are shear-thinning, as already argued in the comments related to the sedimentation of a disk. Note that, in the previous computations, the effective viscosity does not depend on the initial configuration: the dependency with respect to the initial state is lost with sufficient statistical inputs. In practice, long-time behaviour ensures the averaging process, up to the stabilization of the macroscopic properties of the suspension.

\subsubsection{Jeffery orbits for Newtonian and viscoelastic fluids in the non-inertial regime}

Jeffery [34] and Bretherton [9] showed that, in the non-inertial Newtonian regime, ellipsoids of revolution rotate in a linear shear field with a period

$$
T=2 \pi\left(\frac{R_{1}}{R_{2}}+\frac{R_{2}}{R_{1}}\right) G^{-1}
$$

${ }^{1}$ Note that the one-dimensional steady flow relies on the following equations:

$$
\left\{\begin{aligned}
-\mu(1-r) u^{\prime \prime} & =\sigma_{x y}^{\prime} \\
\sigma_{x x} & =\lambda(1+a) \sigma_{x y} u^{\prime} \\
\sigma_{y y} & =-\lambda(1-a) \sigma_{x y} u^{\prime} \\
\sigma_{x y} & =\mu r u^{\prime}+\lambda\left(\frac{1+a}{2} \sigma_{y y}-\frac{1-a}{2} \sigma_{x x}\right) u^{\prime}
\end{aligned}\right.
$$

with the boundary conditions

$$
u\left( \pm L_{y} / 2\right)= \pm S
$$


where $R_{i}$ denote the major and minor axis of an ellipsoid of revolution and $G$ is the shear rate. More precisely, the dynamics of the ellipsoid is determined by the evolution of the orientation $t \mapsto \theta(t)$

$$
\theta(t)=\theta\left(t_{0}\right)+\arctan \left(\frac{R_{1}}{R_{2}} \tan \frac{2 \pi\left(t-t_{0}\right)}{T}\right)
$$

where $t_{0}$ is the initial time. The rotation of an axisymmetric particle in a simple shear flow was studied in detail for particles of arbitrary shapes, provided that the body is modeled as an equivalent ellipsoid. However, non-Newtonian effects are entirely neglected.

The direct simulation allows us to possibly derive Jeffery orbits adapted to the viscoelastic case even for very elongated particles (in which case mesh refinements may be required). Nevertheless, the number of parameters in the Oldroyd model makes it difficult to establish laws for any set of parameters. Data are described in Table 2] referred as experiment [ $\left.\mathrm{F}_{1}\right]$. It is known that two-dimensional simulations grossly over-predict the wall effects seen in three dimensions and, as a consequence, the particle size is carefully chosen so as to limit the wall effects: in particular, hydrodynamic interactions between two particles in the shear flow can be neglected when placed at $(x, y)=\left( \pm \frac{L_{x}}{4}, 0\right)$.

Figures [16 and 17 provide the Jeffery orbits (angle and angular velocity) for different relaxation times and aspect ratios of the particles. These include the regimes $\mathrm{We} \in[0,1]$ (with $\mathrm{Re}=0$ and $\mathrm{E}=+\infty$ ). First, it can be observed that the Newtonian orbits $(\lambda=0)$ are numerically captured when compared to the theoretical ones. Second, it shows that the elastic properties of the fluid tend to increase the period of rotation of the circular or elongated particle. Finally, although the analysis of the sedimenting velocity of a single disk could suggest that the Oldroyd fluid behaves as a pseudo-viscous fluid, with an effective viscosity which would decrease as the Weissenberg number increases, this argument is not supported by the analysis of the Jeffery orbits: in the case of a rotating disk immersed in a shearing Newtonian flow, the Jeffery orbits do not depend on the viscosity of the bulk fluid, see Eq. (17) and (18). As shown by Figure 16, the orbits are modified by the Weissenberg number, which sheds a light on the influence of the elastic properties of the fluid onto its general behavior: numerical simulations evidence the fact that Oldroyd-type fluids with a rigid inclusion do not behave as a pseudo-viscous fluid. Let us comment the influence of the mesh size: interestingly even if the description of the boundary is of poor quality, the dynamics is not essentially affected by this description. Indeed when using mesh refinement inside/near/around the particle (with mesh coarsening far from the particle in order to get tractable computations), the main characteristics of the dynamics (trajectory, period) are numerically preserved. We conclude that, provided the shape of the particle is represented (in particular the elongational properties), basic properties of the dynamics, when averaging outputs in order to get reduced models informations, are captured.

As studied in the sedimentation study, we illustrate the influence of the mesh size and time step of the numerical method on the motion of a particle immersed in a shear flow with a viscoelastic fluid, without inertial effects. The convergence study is based upon the data related to Expriment $\left[\mathrm{F}_{1}\right]$ : the domain is $\left[-\frac{1}{2}, \frac{1}{2}\right] \times\left[-\frac{1}{2}, \frac{1}{2}\right]$, the fluid is viscoelastic $\left(\rho_{\mathrm{f}}=1\right.$, $\mu=1, r=0.5, \lambda=0.5, \delta=0, a=0)$, the particle is a neutrally buoyant ellipse $\left(R_{1} / R_{2}=3, R_{1} \times R_{2}=8.0 \cdot 10^{-4}\right)$ immersed in a shear flow. The regime is non-inertial which means that the total time derivative in the momentum equation is neglected. In Figure 18, the mesh size is fixed to $h=1 / 600$ and we let $\Delta t$ vary between 0.200 (coarse time step) and 0.025. In Figure 8, the time step is fixed to $\Delta t=0.05$ and we let $h$ vary between $1 / 150$ (coarse mesh) and $1 / 750$. In both cases, the convergence is illustrated through a stabilization process of the Jeffery orbit over one rotation period of the particle $t \mapsto \theta(t)$ (where $\theta(t)$ denotes the angular position of the particle at time $t$ ) for small values of $\Delta t$ and $h$. Note that computations related to Expriment $\left[\mathrm{F}_{1}\right]$ and $\left[\mathrm{F}_{2}\right]$ have been made with $\Delta t=0.025$ and $h=1 / 600$, leading to a numerical solution which is close to the reference solution.

\subsubsection{Impact of inertial effects on the Jeffery orbits for Newtonian and viscoelastic fluids}

The effect of inertia on the dynamics of an ellipse suspended in Newtonian shear flow has been investigated in [15]. At small Reynolds number, the behaviour of the particle is periodic. By increasing the Reynolds number, the period of rotation increases, and eventually becomes infinitely large at a critical Reynolds number $\operatorname{Re}_{c}$. At Reynolds numbers above $\operatorname{Re}_{c}$, the particle becomes stationary in a steady-state flow. The transition from a time-periodic to a steady state is through a saddle-node bifurcation.

Data used for the numerical experiments referred as $\left[F_{2}\right]$ are described in Table 22 Then fixing the values for $\rho_{\mathrm{f}}, R_{1}$ and $\mu$, we let $\rho_{\mathrm{f}}=\rho_{B}$ vary between 0 and 4000 in order to study the influence of the Reynolds number over the dynamics of an ellipse, in both Newtonian and viscoelastic cases.

Figures 20, 21 and 22 present the numerical results in a situation which is very close to the one that was studied in [15]. The ratio between the axes of the ellipse is fixed to $R_{1} / R_{2}=2$ and the ratio between the size of the domain 
and the typical length of the ellipse is $\mathrm{L}_{y} / R_{1} \simeq 20$. Figures 20 a) and 21 reproduce the observations that were made in [15]: the period of rotation increases by increasing the Reynolds number, up to a critical value which is between 25.60 and 32.00, which is in agreement with the critical Reynolds number obtained in [15] which about 29.2. When dealing with the viscoelastic case, see Figures $20 \mathrm{~b}$ ) and 22. the Weissenberg number is We $=1$ so that the elasticity number $\mathrm{E}$ varies between 0.02 and $+\infty$. Numerical simulations lead to the similar observation, up to a strong modification of the bifurcation value: the critical Reynolds number is between 12.80 and 19.20 .

Figures 23, 24 and 25 present the numerical results obtained with a higher aspect ratio of the ellipse, $R_{1} / R_{2}=3$. It is shown that the bifurcation value for the Reynolds number, associated to the loss of periodicity of the dynamics, is decreased in both the Newtonian case $\left(\operatorname{Re}_{c}\right.$ is between 9.73 and 11.68) and the viscoelastic case $\left(\operatorname{Re}_{c}\right.$ is between 4.38 and 5.35). Note also that, starting from some initial angular state $\theta(0)=0$, the steady-state is attained more quickly when the aspect ratio of the ellipse becomes important.

\subsubsection{Rheology of dense suspensions in the non-inertial regime}

Numerical simulations of sheared dense suspensions are presented, corresponding to data that are described in Table 2 , referred as experiment $[\mathrm{G}]$, in a regime that corresponds to $[\mathrm{Re}, \mathrm{We}, \mathrm{E}]=[0,2,+\infty]$. In order to ensure the limited influence of the size effect, the rheology of suspensions has been studied with two populations of rigid ellipsoids, which differ from the typical size of ellipsoids:

- population $(*)$ is made of rigid ellipsoids with semi-axes $R_{1} \times R_{2}=2.0 \times 10^{-4} \mathrm{~m}^{2}$;

- population $(* *)$ is made of rigid ellipsoids with semi-axes $R_{1} \times R_{2}=0.9 \times 10^{-4} \mathrm{~m}^{2}$. Thus $(* *)$-ellipsoids are smaller than $(*)$-ellipsoids.

In both cases, the semi-axis ratio $R_{1} / R_{2}=2$. In the range of common solid fraction values, no significant difference was observed from the numerical point of view, when computing the effective viscosity of the suspension. Therefore the numerical simulations will now focus on $(* *)$-ellipsoids.

Note that, on the grounds of the behaviour of the method with a coarse mesh, we expect the macro-quantities of interest (e.g. effective viscosity) are captured provided the shape of the particle is correctly represented (in particular the elongational properties) even if the description of the boundary is rather poor. In that case the use of a unstructured mesh is favoured in order to avoid directional artefacts due to the mesh.

We consider a dense suspension of $N$ rigid $(* *)$-ellipsoids in a viscoelastic fluid, corresponding to dilute to dense solid fractions. Figure 26 presents the evolution of the microstructure composed of the ellipsoids from the initial spatial configuration at different time steps for $N=1296$. The initial spatial distribution is nearly homogeneous: the particles are regularly spaced (except near the artificial periodic boundaries) but the orientations are randomly chosen. In the initial configuration, observe that an empty strip (area with no particle) is designed near the artificial periodic boundary $x= \pm \frac{L_{x}}{2}$; the evolution of the strip (corresponding to no-particle areas) in the first time steps can be observed (see $t=i \Delta t, i \in\{0,10, \ldots, 60\})$ and some time is required to break this particular microstructure until reaching a complete spatial homogeneous configuration with random orientations (see, e.g. $t=250 \Delta t$ ). Thus, combined with the relaxation time of the viscoelastic fluid, this spatial evolution has a strong impact on the effective viscosity.

Figure 27 presents the evolution in time of the effective viscosity of a suspension of $N=1296$ rigid ellipsoids in the Newtonian case and in the viscoelastic case. It exhibits the antagonist effects of the elastic relaxation time and the solid fraction over the effective viscosity.

Direct simulation allows us to quantify the influence of the solid fraction over the effective viscosity reduction. Figures 28 and 29 show the effective viscosity $\mu_{\text {eff. }}$ and the relative effective viscosity $\frac{\mu_{\text {eff. }}}{\mu_{\text {eff }}}$ with respect to the solid fraction, where

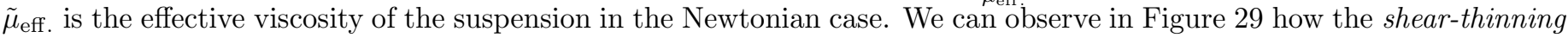
properties of the Oldroyd model are enhanced by the presence of rigid inclusions. In fact, the relative effective viscosity decreases linearly with the solid fraction. Interestingly the slope, which models the sensitivity of the viscosity reduction with respect the solid fraction, depends on the relaxation time of the viscoelastic fluid; it tends to zero when $\lambda$ tends to 0 or $+\infty$, recovering in both cases the Newtonian behaviour of the fluid with respectively $\mu_{\text {eff. }}=\tilde{\mu}_{\text {eff. }}$ and $\mu_{\text {eff. }}=\tilde{\mu}_{\text {eff. }}(1-r)$. The consequences are twofold: the slope does not linearly depend on the relaxation time, and the sensitivity of the viscosity reduction with respect to the solid fraction critically depends on the relaxation time, reaching a maximal value for some intermediate relaxation time. 


\subsubsection{Influence of the material slip parameter}

Computations have been performed for the Johnson-Segalman model with various values of the material slip parameter $a$. In particular, in a regime that corresponds to $[\mathrm{Re}, \mathrm{We}, \mathrm{E}]=[0,0.5,+\infty]$, Figure 30 perfectly captures the behaviour of the effective viscosity for an Oldroyd fluid without rigid entities, see Eq. (16): in particular, upper and lower Maxwell models $(a= \pm 1)$ are not shear-thinning, as it is well-known. Figures 31 and 32 address the same question when dealing with $N=100$ and $N=400$ rigid entities immerged in the fluid. Numerical results suggest the fact that the solid fraction tends to damp the influence of the material slip parameter: as the solid fraction increases, the viscosity reduction becomes important even for values of $|a|$ which are close to 1 . We conjecture that the viscosity of the suspension with a JohnsonSegalman model tends to be close to the one obtained for a corotational model, as the solid fraction increases, but this has to be investigated further.

\section{Conclusion}

In this paper we have presented a numerical strategy to perform the direct simulation of dense 2D suspensions of rigid particles in a viscoelastic fluid. An Oldroyd model is used to describe the viscoelastic properties of the flow and inertial effects may be taken into account. The numerical method is based on a fictitious domain approach, namely a penalty method, that allows the use of standard finite element functional spaces along with fixed and structured meshes. This approach makes it possible to simulate dense suspensions of particles, with a reasonable computational cost, without adding any additional assumption to the model. The complete fluid-structure interaction problem is solved at each time step in a coupled way, what ensures unconditional stability and arbitrary accuracy.

The developed numerical tool allows to intensively study the behavior of viscoelastic suspensions, in different configurations and for different values of the parameters involved in the fluid model.

We have first addressed the sedimentation of a dense suspension in the Newtonian and non-Newtonian regimes, illustrating the impact of inertial effects and elastic properties on the sedimenting velocity. Numerical results show that the latter decreases when increasing the elastic properties of the fluid. Besides, results have shown how inertial effects tend to damp the impact of elasticity on the sedimenting velocity, because they enhance mixing.

We have also addressed the impact of rigid particles on the rheological properties of a viscoelastic fluid, mainly in the non-inertial regime. Our numerical method has been validated in the case where there are no rigid inclusions, by computing the effective viscosity of the viscoelastic fluid after reaching the stationary state, for different values of the elastic relaxation time. The computed values are in agreement with the theoretical ones, with a relative error less than 1\%. The model has then been used to study the influence of the elastic properties of the fluid on the rotation of a rigid particle in a shear flow. The period of rotation increases with the relaxation time, that means that it depends on the Weissenberg number. This shows that a viscoelastic fluid cannot be interpreted as a pseudo-viscous fluid with an effective viscosity that decreases with the Weissenberg number, as could be suggested by the previous numerical experiment, since the period of rotation would then be independent of the viscosity. Finally, we have quantified the dependence of the effective viscosity of a viscoelastic fluid on the solid fraction, for different values of the relaxation time. This study shows how the shear-thining effect of the Oldroyd model is enforced in the presence of rigid particles, and how this effect depends on the relaxation time of the viscoelastic fluid.

Many regimes have not been investigated in our paper, in particular regimes for which the mathematical analysis (existence, uniqueness of solutions) is problematic. Actually numerical simulations may help to understand the behavior of the suspensions in these cases. For instance, the Johnson-Segalman models, $a \neq 0$, require smallness of the data in order to ensure global-in-time solutions. Combined with the high Weissenberg regime, i.e. considering the asymptotic regime $\lambda \rightarrow+\infty$, the solution formally tends to the Newtonian behavior with a viscosity equal to $\mu(1-r)$, where $\mu$ is the solvent viscosity and $r$ is the viscoelastic interpolation parameter in the Oldroyd model. However, this regime is difficult to capture numerically and presents serious stability issues. More adapted numerical methods should be developed to overcome this stability issue. Finally hydrodynamic interactions highly differ in the $2 \mathrm{D}$ and $3 \mathrm{D}$ cases. It would therefore be interesting to investigate numerically $3 D$ suspensions of viscoelastic fluids and for that purpose it would be necessary to propose a different numerical method that is more adapted to the use of iterative methods that circumvent the necessity of assembling the matrices associated to the linear systems to be solved, which are much too large in dimension 3. 


\section{References}

[1] P. Angot, C.-H. Bruneau, and P. Fabrie. A penalization method to take into account obstacles in incompressible viscous flows. Numer. Math., 81(4):497-520, 1999.

[2] J. W. Barrett and E. Süli. Existence of global weak solutions to some regularized kinetic models for dilute polymers. Multiscale Model. Simul., 6(2):506-546, 2007.

[3] M. Belzons, R. Blanc, J. L. Bouillot, and C. Camoin. Viscosité d'une suspension diluée et bidimensionnelle de sphères. C. R. Acad. Sci, Paris II, 292:939-44, 1981.

[4] H. Binous and R. J. Phillips. Dynamic simulation of one and two particles sedimenting in viscoelastic suspensions of \{FENE\} dumbbells. J. Non-Newtonian Fluid Mech., 83(1-2):93-130, 1999.

[5] T. Bodnár and A. Sequeira. Numerical study of the significance of the non-Newtonian nature of blood in steady flow through a stenosed vessel. In Advances in Mathematical Fluid Mechanics. Dedicated to Giovanni Paolo Galdi on the Occasion of his 60th Birthday, pages 83-104. Springer Berlin Heidelberg, 2010.

[6] G. Bossis and J. F. Brady. Dynamic simulation of sheared suspensions. I. General method. J. Chem. Phys., 80:5141$5154,1984$.

[7] J. F. Brady and G. Bossis. Stokesian Dynamics. Ann. Rev. Fluid Mech., 20:111-157, 1988.

[8] H. Brenner and M.E. O'Neill. On the Stokes resistance of multiparticle systems in a linear shear field. Chem. Eng. Sci., 27:1421-1439, 1972.

[9] F. P. Bretherton. The motion of rigid particles in a shear flow at low Reynolds number. J. Fluid Mech., 14:284-304, 1962.

[10] Y. J. Choi, M. A. Hulsen, and H. E. H. Meijer. An extended finite element method for the simulation of particulate viscoelastic flows. J. Non-Newtonian Fluid Mech., 165(11-12):607-624, 2010.

[11] L. Chupin and S. Martin. Stationary Oldroyd model with diffusive stress: mathematical analysis of the model and vanishing diffusion process. J. Non-Newtonian Fluid Mech., 218:27-39, 2015.

[12] P. Constantin and M. Kliegl. Note on global regularity for two-dimensional Oldroyd-B fluids with diffusive stress. Arch. Ration. Mech. Anal., 206(3):725-740, 2012.

[13] G. D'Avino and P.L. Maffettone. Particle dynamics in viscoelastic liquids. J. Non-Newtonian Fluid Mech., 215:80-104, 2015.

[14] G. D'Avino, P.L. Maffettone, F. Greco, and M.A. Hulsen. Viscoelasticity-induced migration of a rigid sphere in confined shear flow. J. Non-Newtonian Fluid Mech., 165(9-10):466-474, 2010.

[15] E.-J. Ding and C. K. Aidun. The dynamics and scaling law for particles suspended in shear flow with inertia. J. Fluid Mech., 423:317-344, 2000.

[16] J. Feng, P.Y. Huang, and D.D. Joseph. Dynamic simulation of sedimentation of solid particles in an Oldroyd-B fluid. J. Non-Newtonian Fluid Mech., 63(1):63-88, 1996.

[17] A. F. Fortes, D. D. Joseph, and T. S. Lundgren. Nonlinear mechanics of fluidization of beds of spherical particles. $J$. Fluid Mech., 177:407-483, 1987.

[18] R. Glowinski, T.-W. Pan, T. I. Hesla, D. D. Joseph, and J. Periaux. A distributed Lagrange multiplier/fictitious domain method for the simulation of flow around moving rigid bodies: application to particulate flow. Comput. Methods Appl. Mech. Engrg., 184(2-4):241-267, 2000.

[19] R. Glowinski, T. W. Pan, T. I. Hesla, D. D. Joseph, and J. Périaux. A fictitious domain approach to the direct numerical simulation of incompressible viscous flow past moving rigid bodies: application to particulate flow. $J$. Comput. Phys., 169(2):363-426, 2001. 
[20] N. Goyal and J.J. Derksen. Direct simulations of spherical particles sedimenting in viscoelastic fluids. J. NonNewtonian Fluid Mech., 183-184:1-13, 2012.

[21] C. Guillopé and J.-C. Saut. Existence results for the flow of viscoelastic fluids with a differential constitutive law. Nonlinear Anal., 15(9):849-869, 1990.

[22] J. Hao, T. W. Pan, R. Glowinski, and D. D. Joseph. A fictitious domain/distributed Lagrange multiplier method for the particulate flow of Oldroyd-B fluids: A positive definiteness preserving approach. J. Non-Newtonian Fluid Mech., 156(1-2):95-111, 2009.

[23] J. Happel and H. Brenner. Low Reynolds number hydrodynamics. Martinus Nijho, Dordrecht, 1986 (reprint of the 1963 edition).

[24] M. Hillairet. Lack of collision between solid bodies in a 2D incompressible viscous flow. Comm. Partial Differential Equations, 32(7-9):1345-1371, 2007.

[25] M. Hillairet, A. Lozinski, and M. Szopos. On discretization in time in simulations of particulate flows. Discrete Contin. Dyn. Syst. Ser. B, 15(4):935-956, 2011.

[26] M. Hillairet and T. Takahashi. Collisions in three-dimensional fluid structure interaction problems. SIAM Journal on Mathematical Analysis, 40(6):2451-2477, 2009.

[27] M. Hillairet and T. Takahashi. Blow up and grazing collision in viscous fluid solid interaction systems. Annales de l'Institut Henri Poincare (C) Non Linear Analysis, 27(1):291-313, 2010.

[28] H. H. Hu. Direct simulation of flows of solid-liquid mixtures. Int. J. Multiphase Flow, 22:335-352, 1996.

[29] H. H. Hu, D. D. Joseph, and M. J. Crochet. Direct simulation of fluid particle motions. Theor. Comput. Fluid Dynam., 3(5):285-306, 1992.

[30] P. Y. Huang, J. Feng, H. H. Hu, and D. D. Joseph. Direct simulation of the motion of solid particles in Couette and Poiseuille flows of viscoelastic fluids. J. Fluid Mech., 343:73-94, 1997.

[31] P. Y. Huang, H. H. Hu, and D. D. Joseph. Direct simulation of the sedimentation of elliptic particles in Oldroyd-B fluids. J. Fluid Mech., 362:297-325, 1997.

[32] W. R. Hwang, M. A. Hulsen, and H. E.H. Meijer. Direct simulation of particle suspensions in sliding bi-periodic frames. J. Comput. Phys., 194(2):742-772, 2004.

[33] J. Janela, A. Lefebvre, and B. Maury. A penalty method for the simulation of fluid-rigid body interaction. In CEMRACS 2004-mathematics and applications to biology and medicine, volume 14 of ESAIM Proc., pages 115-123. EDP Sci., Les Ulis, 2005.

[34] G. B. Jeffery. The motion of ellipsoidal particles immersed in a viscous fluid. Proc. R. Soc. Lond. A, 102:161-179, 1922.

[35] A. A. Johnson and T. E. Tezduyar. Simulation of multiple spheres falling in a liquid-filled tube. Comput. Methods Appl. Mech. Engrg., 134(3-4):351-373, 1996.

[36] D. D. Joseph. Fluid Dynamics of Viscoelastic Liquids, volume 84 of Applied Mathematical Sciences. Springer, New York, 1998. $754 \mathrm{pp}$.

[37] D.D. Joseph, Y.J. Liu, M. Poletto, and J. Feng. Aggregation and dispersion of spheres falling in viscoelastic liquids. J. Non-Newtonian Fluid Mech., 54:45-86, 1994.

[38] I. M. Krieger and T. J. Dougherty. A mechanism for non-Newtonian flow in suspensions of rigid spheres. T. Soc. Rheol., 3(1):137-152, 1959.

[39] A. Lefebvre. Fluid-particle simulations with FreeFem++. In Paris-Sud Working Group on Modelling and Scientific Computing 2006-2007, volume 18 of ESAIM Proc., pages 120-132. EDP Sci., Les Ulis, 2007. 
[40] A. Lefebvre. Numerical simulation of gluey particles. M2AN Math. Model. Numer. Anal., 43(1):53-80, 2009.

[41] G. Li, G. H. McKinley, and A. M. Ardekani. Dynamics of particle migration in channel flow of viscoelastic fluids. $J$. Fluid Mech., 785:486-505, 122015.

[42] P. L. Lions and N. Masmoudi. Global solutions for some Oldroyd models of non-Newtonian flows. Chinese Ann. Math. Ser. B, 21(2):131-146, 2000.

[43] B. Maury. Direct simulations of 2D fluid-particle flows in biperiodic domains. J. Comput. Phys., 156(2):325-351, 1999.

[44] B. Maury. A time-stepping scheme for inelastic collisions. Numerical handling of the nonoverlapping constraint. Numer. Math., 102(4):649-679, 2006.

[45] L. Molinet and R. Talhouk. On the global and periodic regular flows of viscoelastic fluids with a differential constitutive law. NoDEA Nonlinear Differential Equations Appl., 11(3):349-359, 2004.

[46] N. A. Patankar and H.H. Hu. Rheology of a suspension of particles in viscoelastic fluids. J. Non-Newtonian Fluid Mech., 96(3):427-443, 2001.

[47] N.A. Patankar, P. Singh, D.D. Joseph, R. Glowinski, and T-W. Pan. A new formulation for the distributed Lagrange multiplier/fictitious domain method for particulate flows. Int. J. Multiphase Flow, 26:1509-1524, 2000.

[48] T. N. Randrianarivelo, G. Pianet, S. Vincent, and J. P. Caltagirone. Numerical modelling of solid particle motion using a new penalty method. Int. J. Numer. Meth. Fluids, 47(10-11):1245-1251, 2005.

[49] M. Renardy. Existence of slow steady flows of viscoelastic fluids with differential constitutive equations. Z. Angew. Math. Mech., 65(9):449-451, 1985.

[50] J.-C. Saut. Lectures on the mathematical theory of viscoelastic fluids. Lect. Anal. Nonlinear Partial Differ. Equ., 3:325-393, 2013.

[51] H. M. Schaink, J. J. M. Slot, R. J. J. Jongschaap, and J. Mellema. The rheology of systems containing rigid spheres suspended in both viscous and viscoelastic media, studied by Stokesian dynamics simulations. J. Rheol., 44(3):473498,2000 .

[52] P. Singh, D.D. Joseph, T.I. Hesla, R. Glowinski, and T.-W. Pan. A distributed lagrange multiplier/fictitious domain method for viscoelastic particulate flows. J. Non-Newtonian Fluid Mech., 91(2-3):165-188, 2000.

[53] Z. Yu and X. Shao. A direct forcing fictitious domain method for particulate flows. J. Comput. Phys., 227(1):292-314, 2007.

[54] Z. Yu, A. Wachs, and Y. Peysson. Numerical simulation of particle sedimentation in shear-thinning fluids with a fictitious domain method. J. Non-Newtonian Fluid Mech., 136(2-3):126-139, 2006. 


\begin{tabular}{|c|c|c|c|c|c|}
\hline & & {$\left[\mathrm{A}_{1}\right]$} & {$\left[\mathrm{A}_{2}\right]$} & {$[\mathrm{B}]$} & {$[\mathrm{C}]$} \\
\hline \multicolumn{6}{|l|}{ Domain } \\
\hline $\mathrm{L}_{x}$ & {$[\mathrm{~m}]$} & 2 & 2 & 2 & 2 \\
\hline $\mathrm{L}_{y}$ & {$[\mathrm{~m}]$} & 6 & 6 & 10 & 2 \\
\hline \multicolumn{6}{|l|}{ Fluid } \\
\hline$\mu$ & {$[\mathrm{Pa} \cdot \mathrm{s}]$} & $1.0 \cdot 10^{-2}$ & $1.0 \cdot 10^{-2}$ & $1.0 \cdot 10^{-2}$ & $1.0 \cdot 10^{-2}$ \\
\hline$\rho_{\mathrm{f}}$ & {$\left[\mathrm{kg} \cdot \mathrm{m}^{-3}\right]$} & 0.1 & {$[0.1-0.5]$} & 0.50 & 1.00 \\
\hline$r$ & {$[-]$} & 0.5 & {$[0,0.5]$} & $\{0.0,0.5\}$ & $\{0.0,0.5\}$ \\
\hline$\lambda$ & & 1.0 & {$[0.0-1.0]$} & $\{0.0,0.1\}$ & $\{0.0,1.0\}$ \\
\hline$\delta$ & {$\left[\mathrm{m}^{2}\right]$} & 0. & 0 & $\begin{array}{c}{[0.0,0.1]} \\
0 .\end{array}$ & $\begin{array}{c}{[0.0,1.0\}} \\
0 .\end{array}$ \\
\hline$a$ & {$[-]^{1}$} & 0 . & 0 . & 0 . & 0 . \\
\hline \multicolumn{6}{|c|}{ Boundary conditions } \\
\hline$x= \pm \frac{L_{x}}{r^{2}}$ & & $\mathbf{u}=\mathbf{0}$ & $\mathbf{u}=\mathbf{0}$ & $\mathbf{u}=\mathbf{0}$ & $\mathbf{u}=\mathbf{0}$ \\
\hline$y= \pm \frac{L_{y}}{2}$ & & $\mathbf{u}=\mathbf{0}$ & $\mathbf{u}=\mathbf{0}$ & $\mathbf{u}=\mathbf{0}$ & $\mathbf{u}=\mathbf{0}$ \\
\hline \multicolumn{6}{|l|}{ Particle } \\
\hline$N$ & {$[-]$} & 1 & 1 & 2 & 228 \\
\hline$R_{1}$ & {$[\mathrm{~m}]$} & 0.125 & 0.125 & 0.125 & 0.040 \\
\hline$R_{2}$ & {$[\mathrm{~m}]$} & 0.125 & 0.125 & 0.125 & 0.040 \\
\hline$\rho_{B}$ & {$\left[\mathrm{~kg} \cdot \mathrm{m}^{-3}\right]$} & $1+\rho_{\mathrm{f}}$ & $1+\rho_{\mathrm{f}}$ & 1.5 & 50.0 \\
\hline \multicolumn{6}{|l|}{ Gravity } \\
\hline$g$ & {$\left[\mathrm{~m} \cdot \mathrm{s}^{-2}\right]$} & 10.0 & 10.0 & 10.0 & 10.0 \\
\hline \multicolumn{6}{|l|}{ Regimes } \\
\hline $\operatorname{Re}$ & {$[-]$} & $0^{*}$ & {$\left[0^{*}-60\right]$} & $\{75,150\}$ & 2 \\
\hline We & {$[-]$} & 64 & {$[0-80]$} & $\{0,20\}$ & $\{0,12\}$ \\
\hline \multicolumn{6}{|c|}{ Discretization } \\
\hline$\Delta t$ & {$[\mathrm{~s}]$} & $5.0 \cdot 10^{-3}$ & $5.0 \cdot 10^{-3}$ & $5.0 \cdot 10^{-3}$ & $1.0 \cdot 10^{-2}$ \\
\hline$h$ & {$[\mathrm{~m}]$} & $2.0 \cdot 10^{-2}$ & $2.0 \cdot 10^{-2}$ & $2.0 \cdot 10^{-2}$ & $2.0 \cdot 10^{-2}$ \\
\hline
\end{tabular}

Table 1: Physical and numerical parameters for the sedimentation study. ${ }^{*}$ Note that non-inertial regimes, $\operatorname{Re}=0$, are computed by neglecting the total time derivative in the momentum equation. 


\begin{tabular}{|c|c|c|c|c|c|c|c|}
\hline & & {$[\mathrm{D}]$} & {$[\mathrm{E}]$} & {$\left[\mathrm{F}_{1}\right]$} & {$\left[\mathrm{F}_{2}\right]$} & {$[\mathrm{G}]$} & {$[\mathrm{H}]$} \\
\hline \multicolumn{8}{|l|}{ Domain } \\
\hline $\mathrm{L}_{x}$ & {$[\mathrm{~m}]$} & 1 & 1 & 1 & 1 & 1 & 1 \\
\hline $\mathrm{L}_{y}^{\infty}$ & [m] & 1 & 1 & 1 & 1 & 1 & 1 \\
\hline \multicolumn{8}{|l|}{ Fluid } \\
\hline$\mu$ & {$[\mathrm{Pa} \cdot \mathrm{s}]$} & $1.0 \cdot 10^{+0}$ & $1.0 \cdot 10^{+0}$ & $1.0 \cdot 10^{+0}$ & $1.0 \cdot 10^{+0}$ & $1.0 \cdot 10^{+0}$ & $1.0 \cdot 10^{+0}$ \\
\hline$\rho_{\mathrm{f}}$ & {$\left[\mathrm{kg} \cdot \mathrm{m}^{-3}\right]$} & 1 & 1 & 1 & {$[1-4000]$} & 1 & 1 \\
\hline$r$ & {$[-]$} & 0.0 & 0.5 & 0.5 & $\{0.0,0.5\}$ & 0.5 & 0.5 \\
\hline$\lambda$ & & 0.0 & {$[0.0-1.0]$} & {$[0.0-0.5]$} & $\{0.0,0.5\}$ & {$[0.0-1.0]$} & 0.25 \\
\hline$\delta$ & {$\left[\mathrm{m}^{2}\right]$} & 0. & 0 & 0 & 0 & 0 & 0. \\
\hline$a$ & {$[-]$} & 0. & 0. & 0. & 0. & 0. & $a \in[-1,1]$ \\
\hline \multicolumn{8}{|c|}{ Boundary conditions } \\
\hline$x= \pm \frac{L_{x}}{2}$ & & periodicity & periodicity & periodicity & periodicity & periodicity & periodicity \\
\hline$y= \pm \frac{L_{y}}{2}$ & & $\mathbf{u}= \pm(1,0)$ & $\mathbf{u}= \pm(1,0)$ & $\mathbf{u}= \pm(1,0)$ & $\mathbf{u}= \pm(1,0)$ & $\mathbf{u}= \pm(1,0)$ & $\mathbf{u}= \pm(1,0)$ \\
\hline \multicolumn{8}{|l|}{ Particle } \\
\hline$N$ & {$[-]$} & {$[5-200]$} & 0 & 1 & 1 & {$[0-1444]$} & $\{0,100,400\}$ \\
\hline$R_{1} \times R_{2}$ & {$\left[\mathrm{~m}^{2}\right]$} & $2.0 \cdot 10^{-4}$ & & $8.0 \cdot 10^{-4}$ & $8.0 \cdot 10^{-4}$ & $0.9 \cdot 10^{-4}$ & $0.9 \cdot 10^{-4}$ \\
\hline$R_{1} / R_{2}$ & & 1 & & {$[1-3]$} & $\{2,3\}$ & 2 & 2 \\
\hline$\rho_{B}$ & {$\left[\mathrm{~kg} \cdot \mathrm{m}^{-3}\right]$} & $\rho_{\mathrm{f}}$ & & $\rho_{\mathrm{f}}$ & $\rho_{\mathrm{f}}$ & $\rho_{\mathrm{f}}$ & $\rho_{\mathrm{f}}$ \\
\hline \multicolumn{8}{|l|}{ Gravity } \\
\hline$g$ & {$\left[\mathrm{~m} \cdot \mathrm{s}^{-2}\right]$} & 0.0 & 0.0 & 0.0 & 0.0 & 0.0 & 0.0 \\
\hline \multicolumn{8}{|c|}{ Regimes } \\
\hline Re & {$[-]$} & $0^{*}$ & $0^{*}$ & $0^{*}$ & {$\left[0^{*}-60\right]$} & $0^{*}$ & $0^{*}$ \\
\hline We & {$[-]$} & 0 & {$[0-2]$} & {$[0-1]$} & $\{0,1\}$ & {$[0-2]$} & 0.5 \\
\hline \multicolumn{8}{|c|}{ Discretization } \\
\hline$\Delta t$ & {$[\mathrm{~s}]$} & $3.0 \cdot 10^{-2}$ & $3.0 \cdot 10^{-2}$ & $2.5 \cdot 10^{-2}$ & $2.5 \cdot 10^{-2}$ & $3.0 \cdot 10^{-2}$ & $3.0 \cdot 10^{-2}$ \\
\hline$h$ & {$[\mathrm{~m}]$} & $5.0 \cdot 10^{-3}$ & $1.0 \cdot 10^{-2}$ & $1.5 \cdot 10^{-3}$ & $1.5 \cdot 10^{-3}$ & $5.0 \cdot 10^{-3}$ & $5.0 \cdot 10^{-3}$ \\
\hline
\end{tabular}

Table 2: Physical and numerical parameters for the shear study. ${ }^{*}$ Note that non-inertial regimes, $\operatorname{Re}=0$, are computed by neglecting the total time derivative in the momentum equation. 


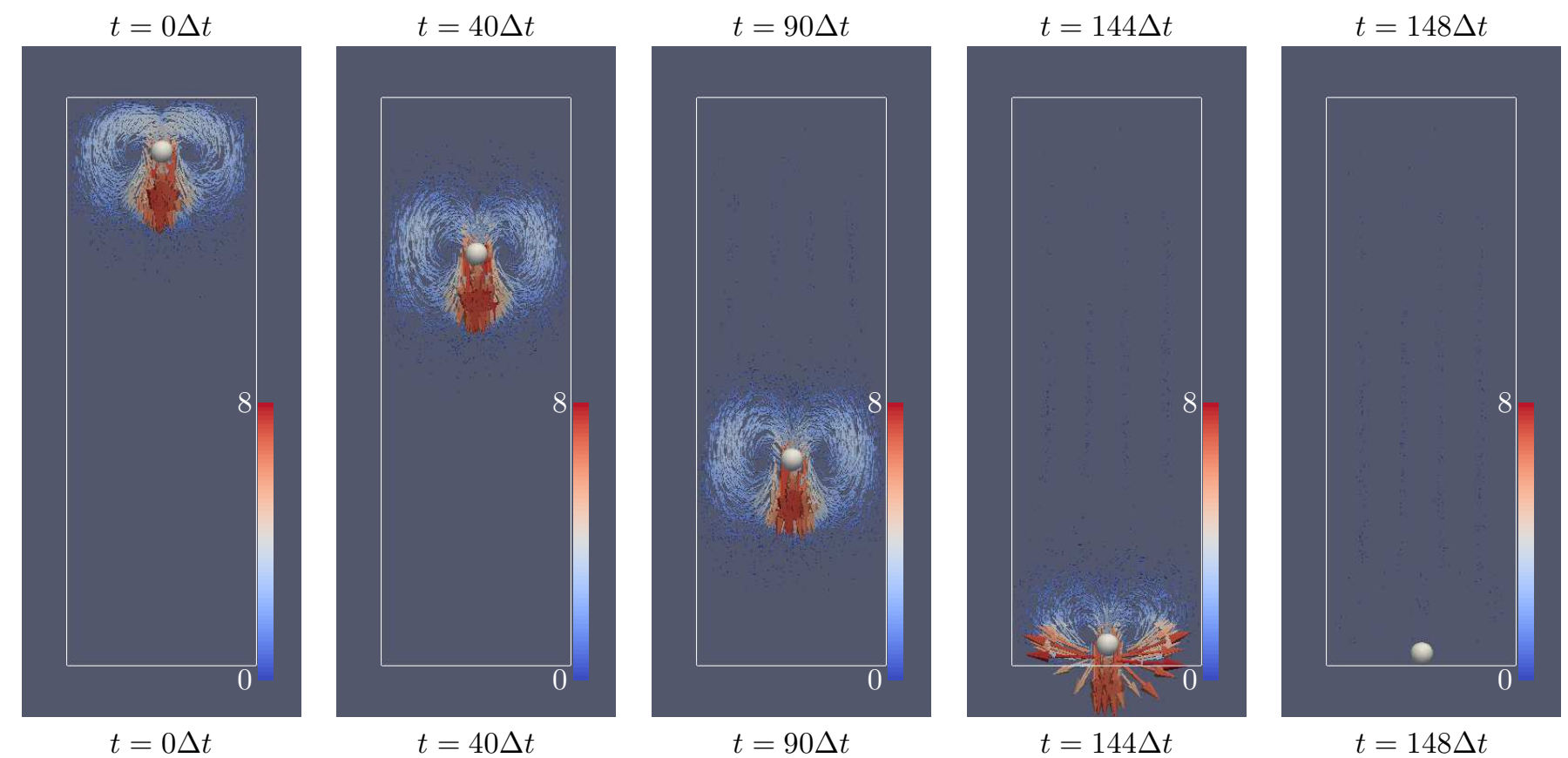

Figure 3: Experiment $\left[\mathrm{A}_{1}\right]$. Sedimenting disk in a viscoelastic fluid in a non-inertial regime: velocity field $\mathbf{u}$ near the disk. 
$t=0 \Delta t$

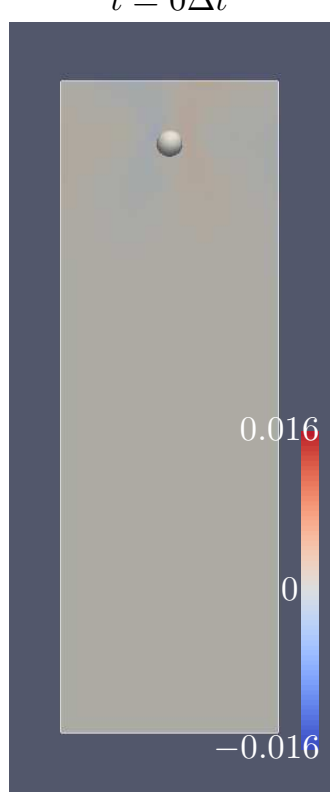

Ni

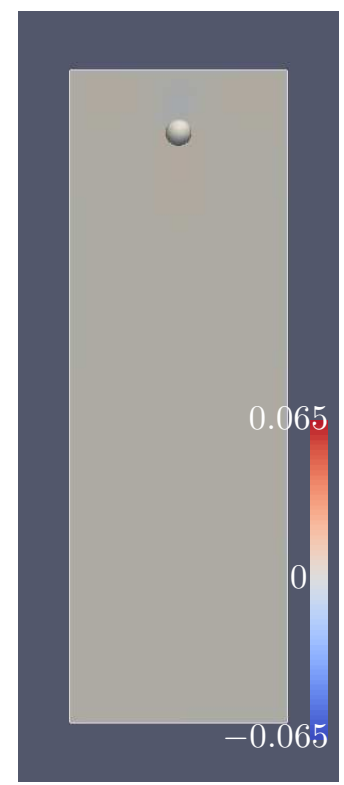

$t=40 \Delta t$

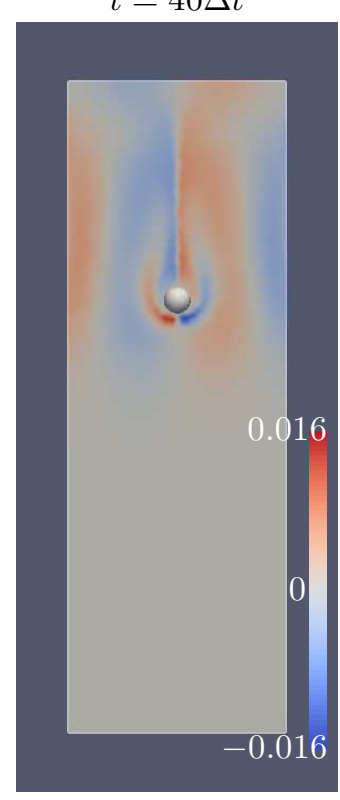

$t=90 \Delta t$
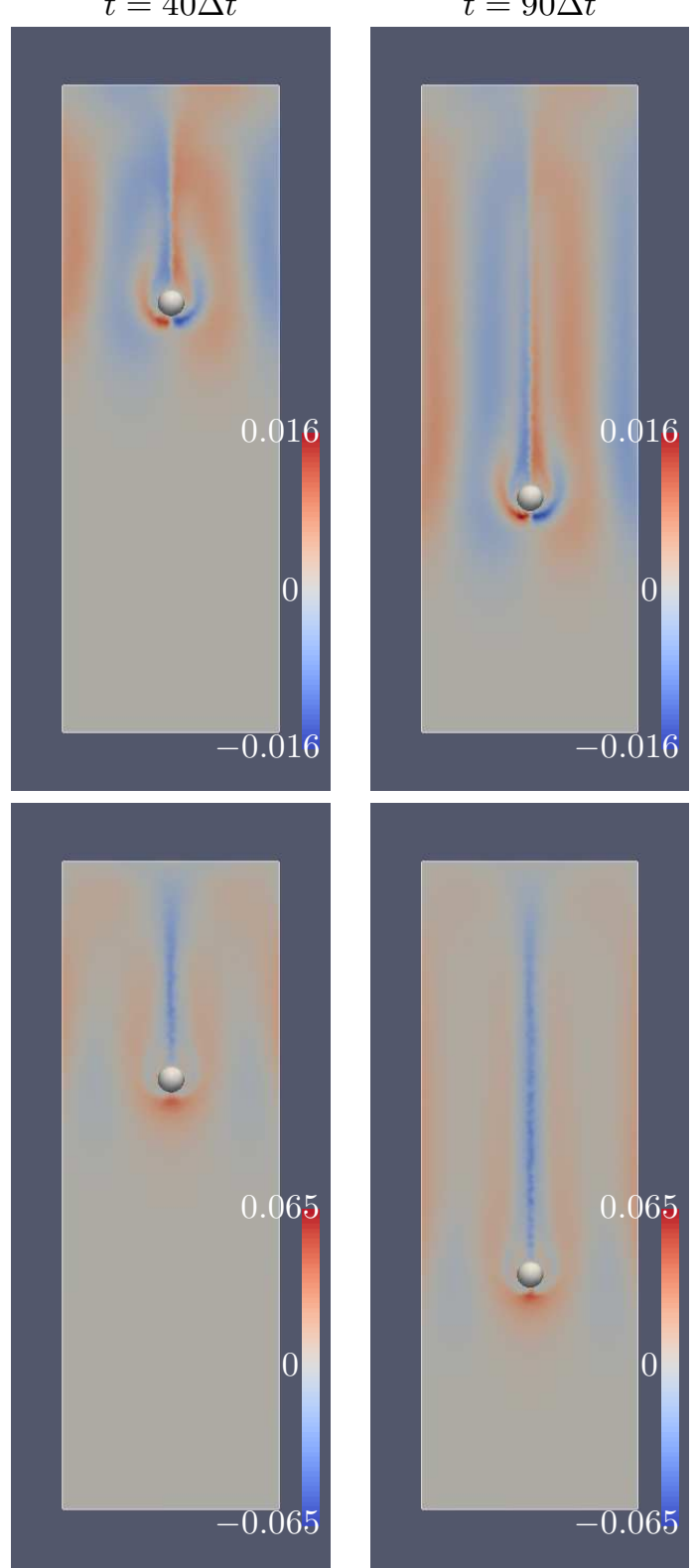

$t=150 \Delta t$

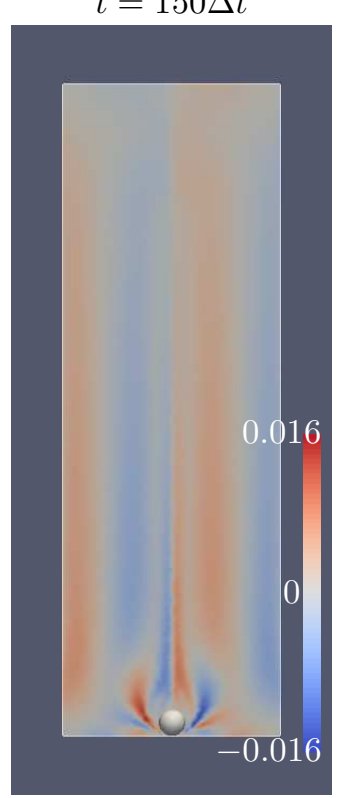

$t=300 \Delta t$
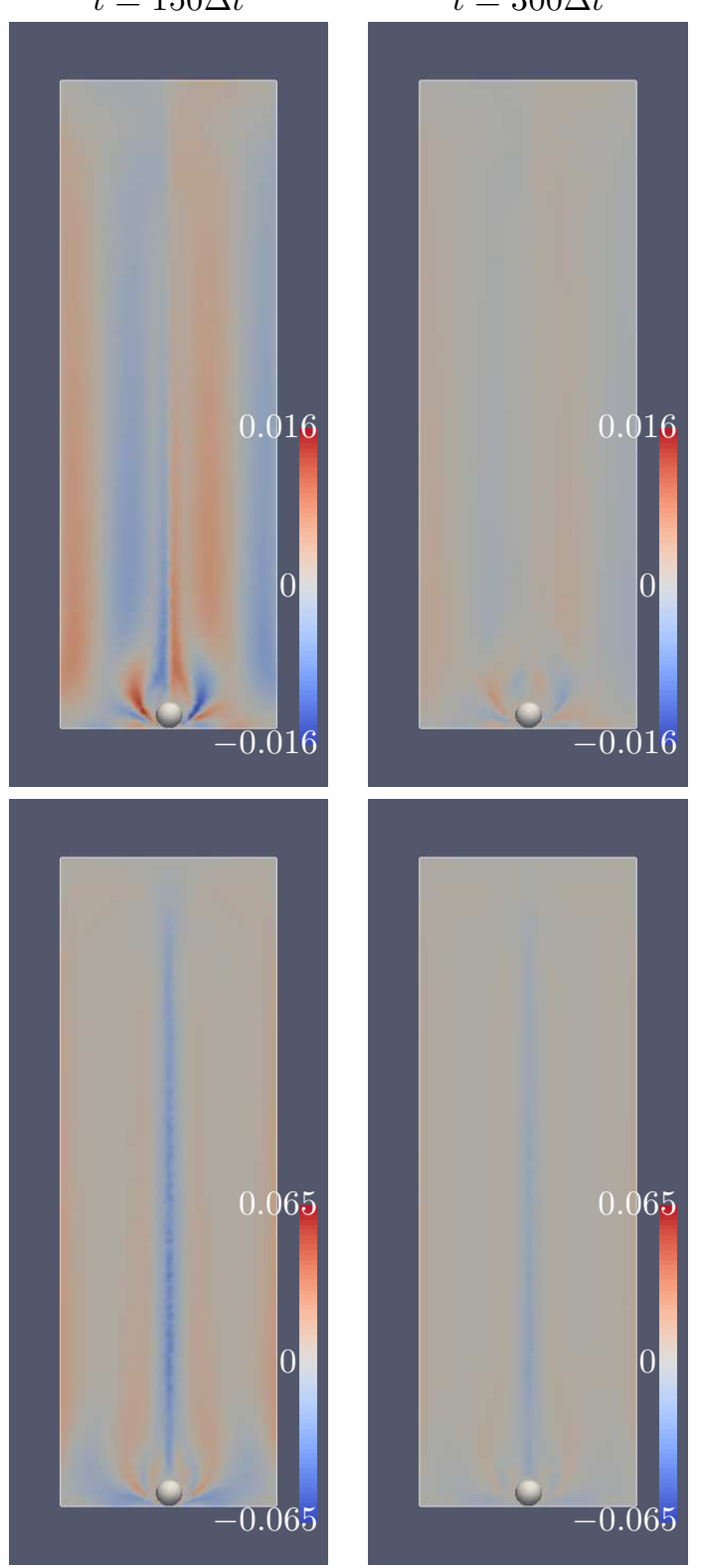

Figure 4: Experiment $\left[\mathrm{A}_{1}\right]$. Sedimenting disk in a viscoelastic fluid in a non-inertial regime: a) shear component $\sigma_{x y}$, b) elongational component $\sigma_{x x}-\sigma_{y y}$ of the elastic tensor at different time steps. 

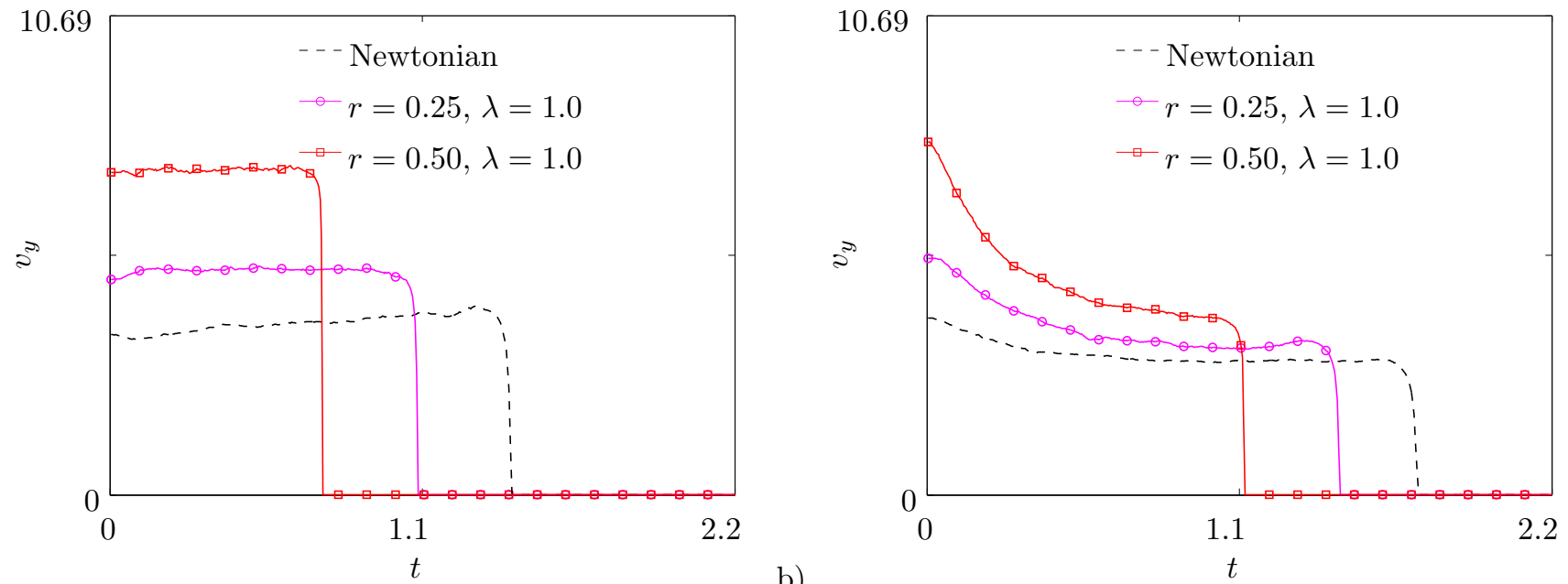

a)

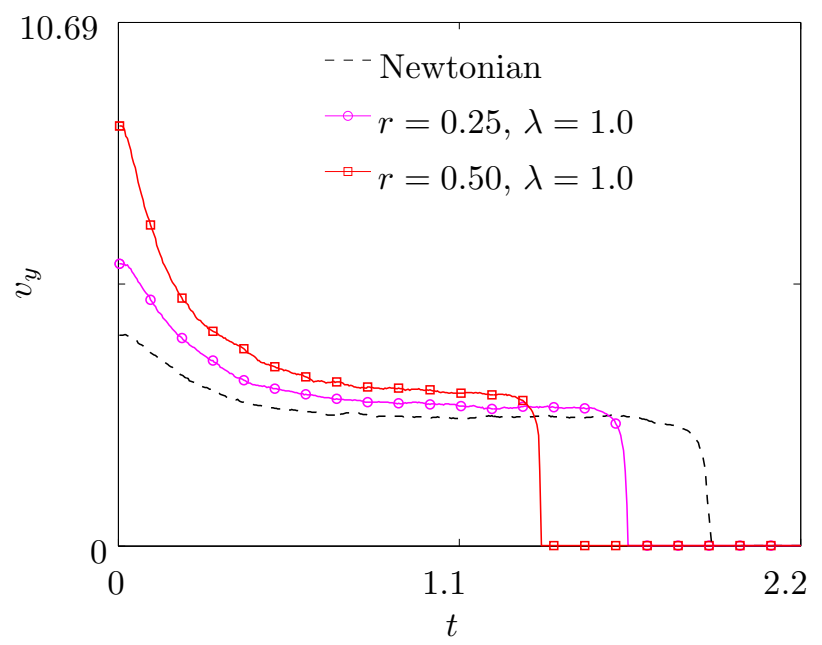

b)

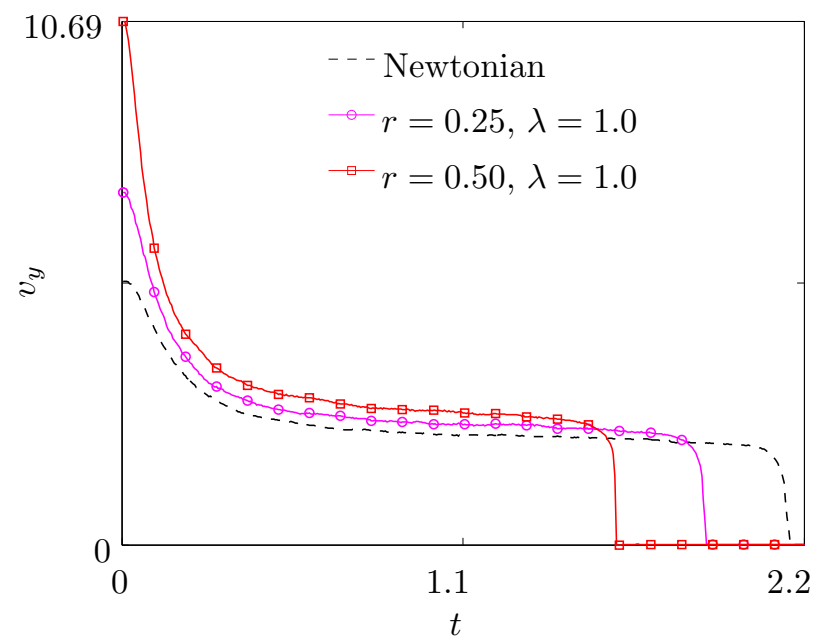

Figure 5: Experiment $\left[\mathrm{A}_{2}\right]$. Sedimentation of a disk in a viscoelastic fluid: influence of the retardation parameter and inertial effects over the evolution of the sedimenting velocity. a) $R e=0$ (non-inertial regime obtained by neglecting the total time derivative in the momentum equation), b) $R e=12.5$ (obtained with $\rho_{\mathrm{f}}=0.1$ ), c) $\operatorname{Re}=25$ (obtained with $\rho_{\mathrm{f}}=0.2$ ), d) $\operatorname{Re}=60$ (obtained with $\rho_{\mathrm{f}}=0.5$ ). Note that the Newtonian case is obtained with $\lambda=0.0$ and $r=0.0$ (but any value for $r$ is valid). 

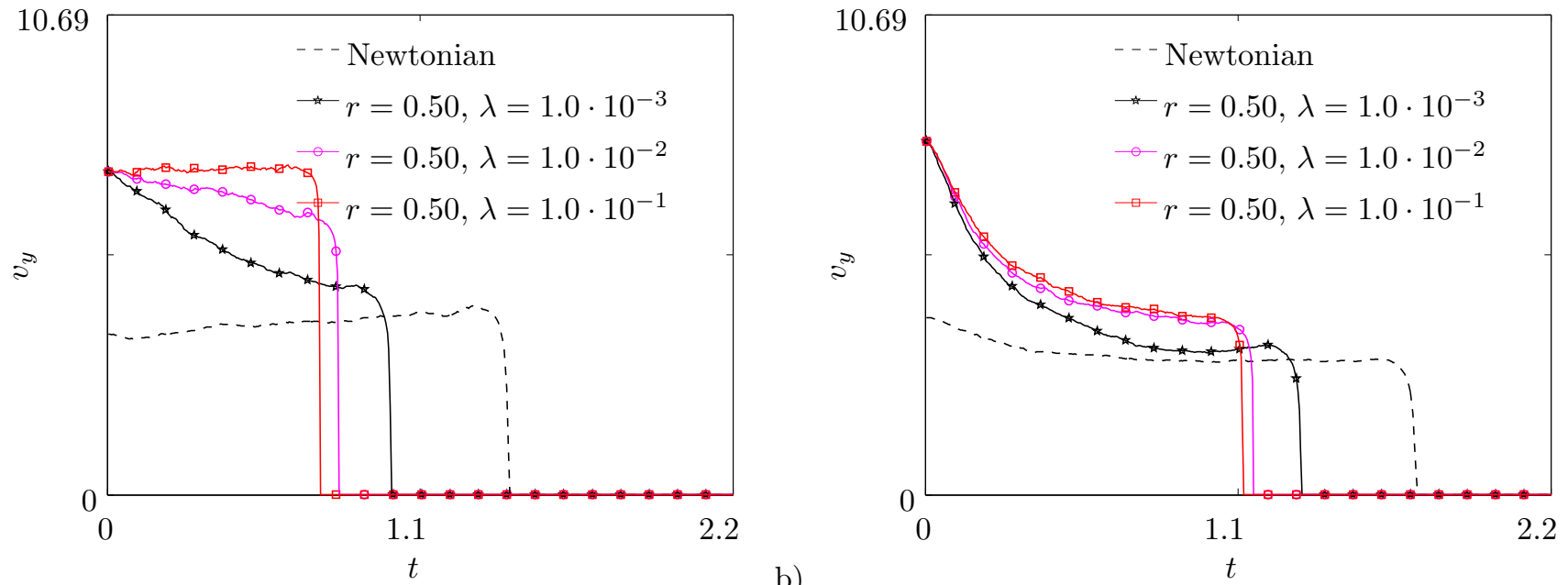

a)

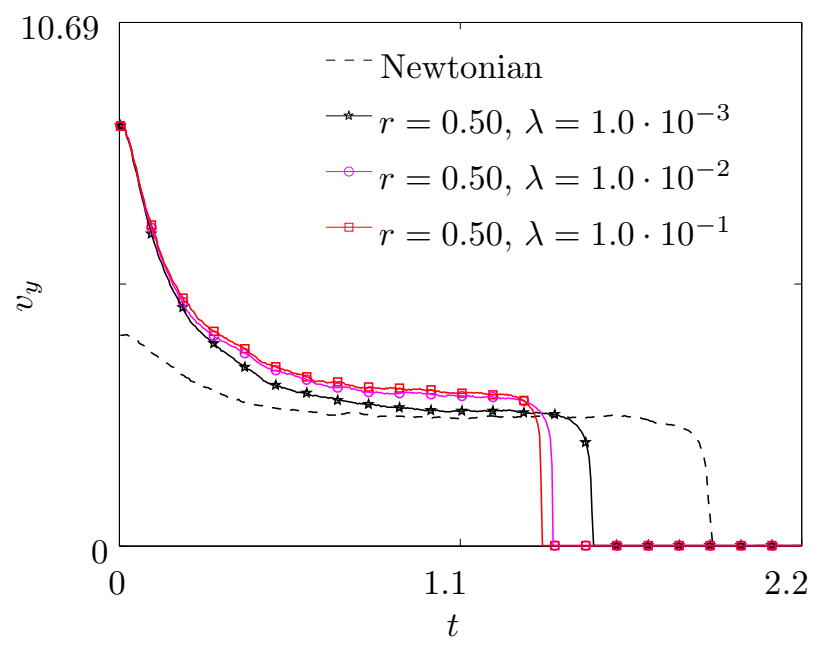

b)

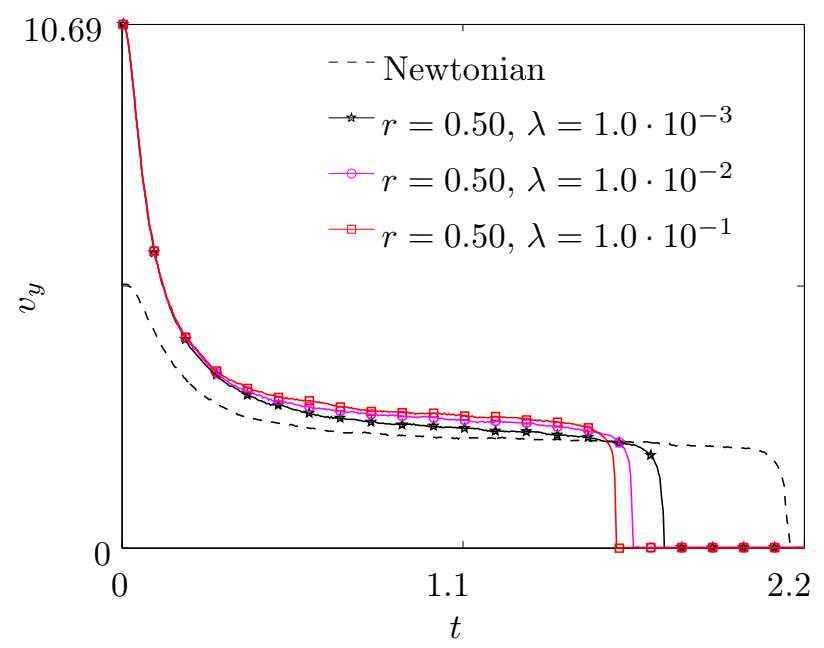

Figure 6: Experiment $\left[\mathrm{A}_{2}\right]$. Sedimentation of a disk: influence of the relaxation time and the inertial effects over the evolution of the sedimenting velocity. a) $\mathrm{Re}=0$ (non-inertial regime obtained by neglecting the total time derivative in the momentum equation), b) $\operatorname{Re}=12.5$ (obtained with $\rho_{\mathrm{f}}=0.1$ ), c) $\operatorname{Re}=25$ (obtained with $\rho_{\mathrm{f}}=0.2$ ), d) $\operatorname{Re}=60$ (obtained with $\rho_{\mathrm{f}}=0.5$ ). Note that the Newtonian case is obtained with $\lambda=0.0$ and $r=0.0$ (but any value for $r$ is valid). 

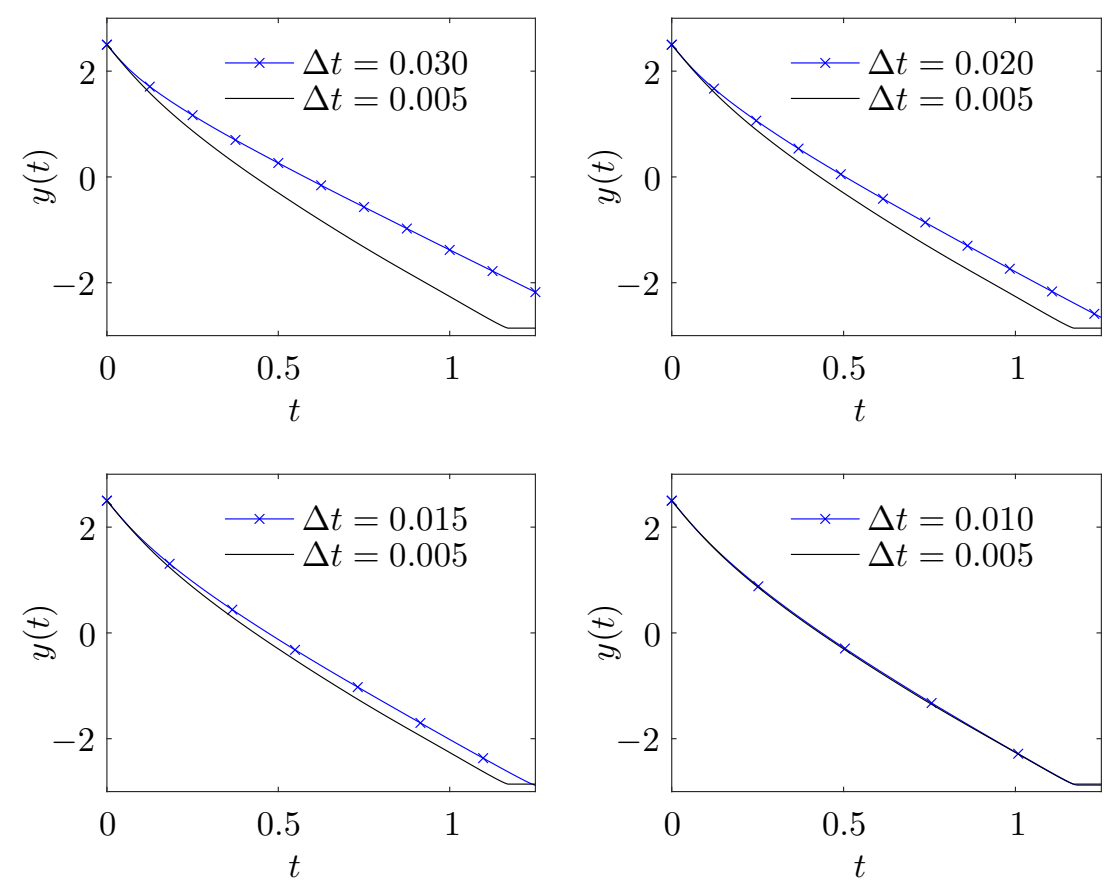

Figure 7: Influence of the time step $\Delta t$ on the position of a sedimenting sphere in a viscoelastic fluid.
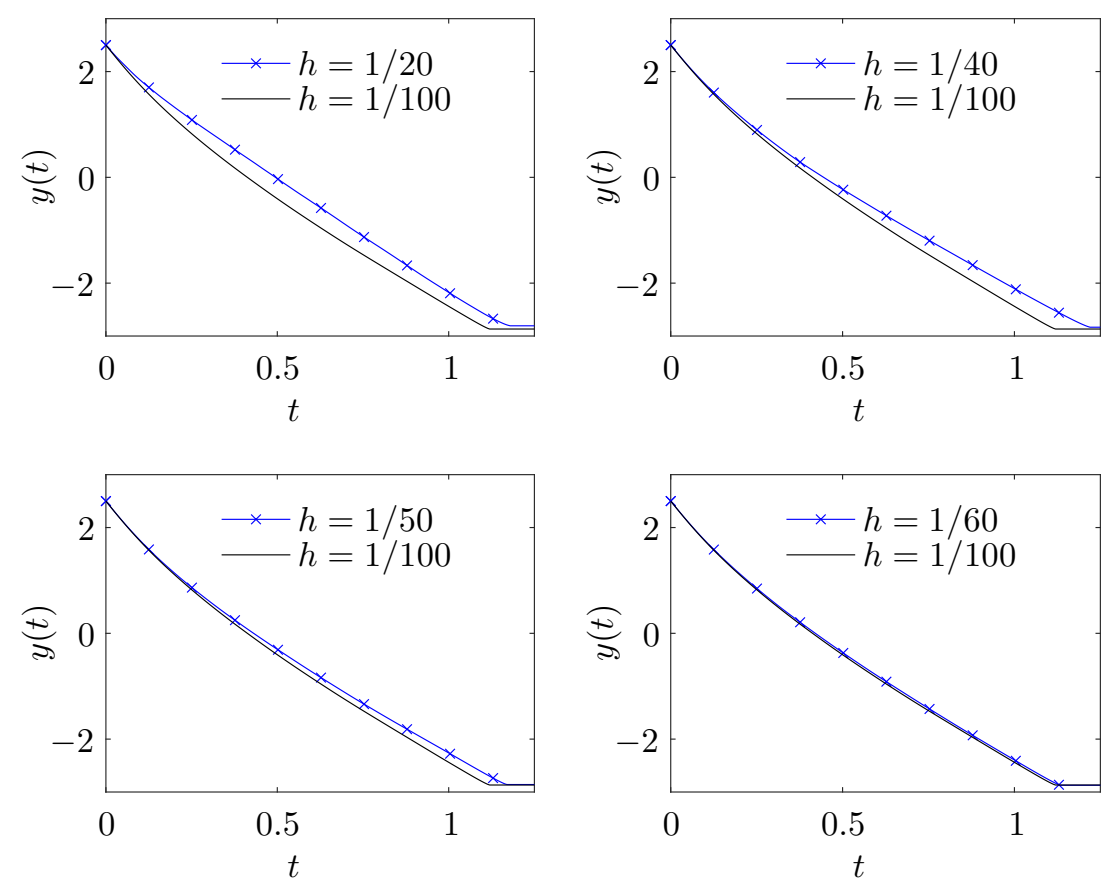

Figure 8: Influence of the mesh size $h$ on the position of a sedimenting sphere in a viscoelastic fluid. 


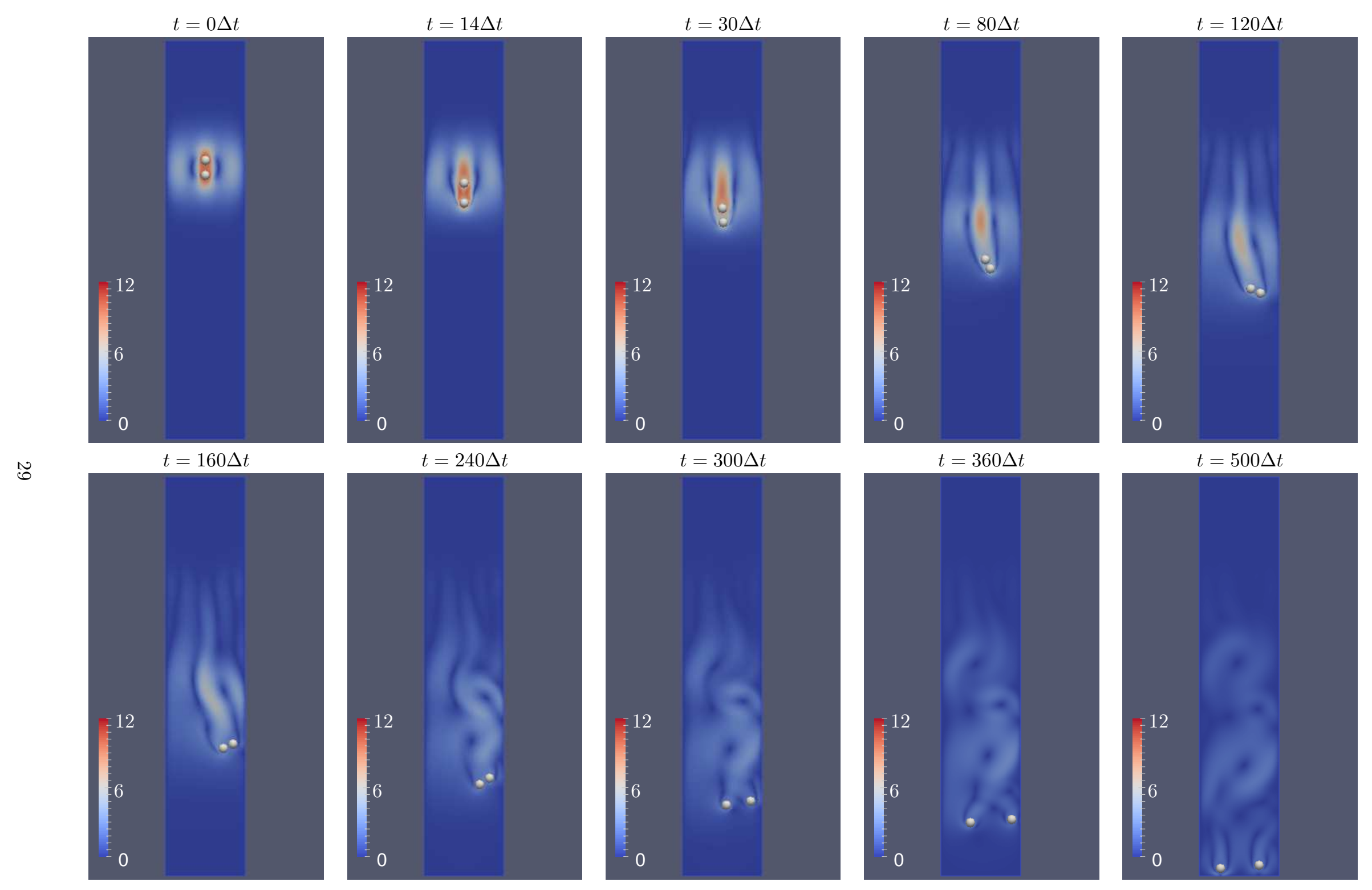

Figure 9: Experiment [B]. Drafting and kissing and tumbling: sedimenting disks in a Newtonian fluid and modulus $\|\mathbf{u}\|$ of the velocity field. 


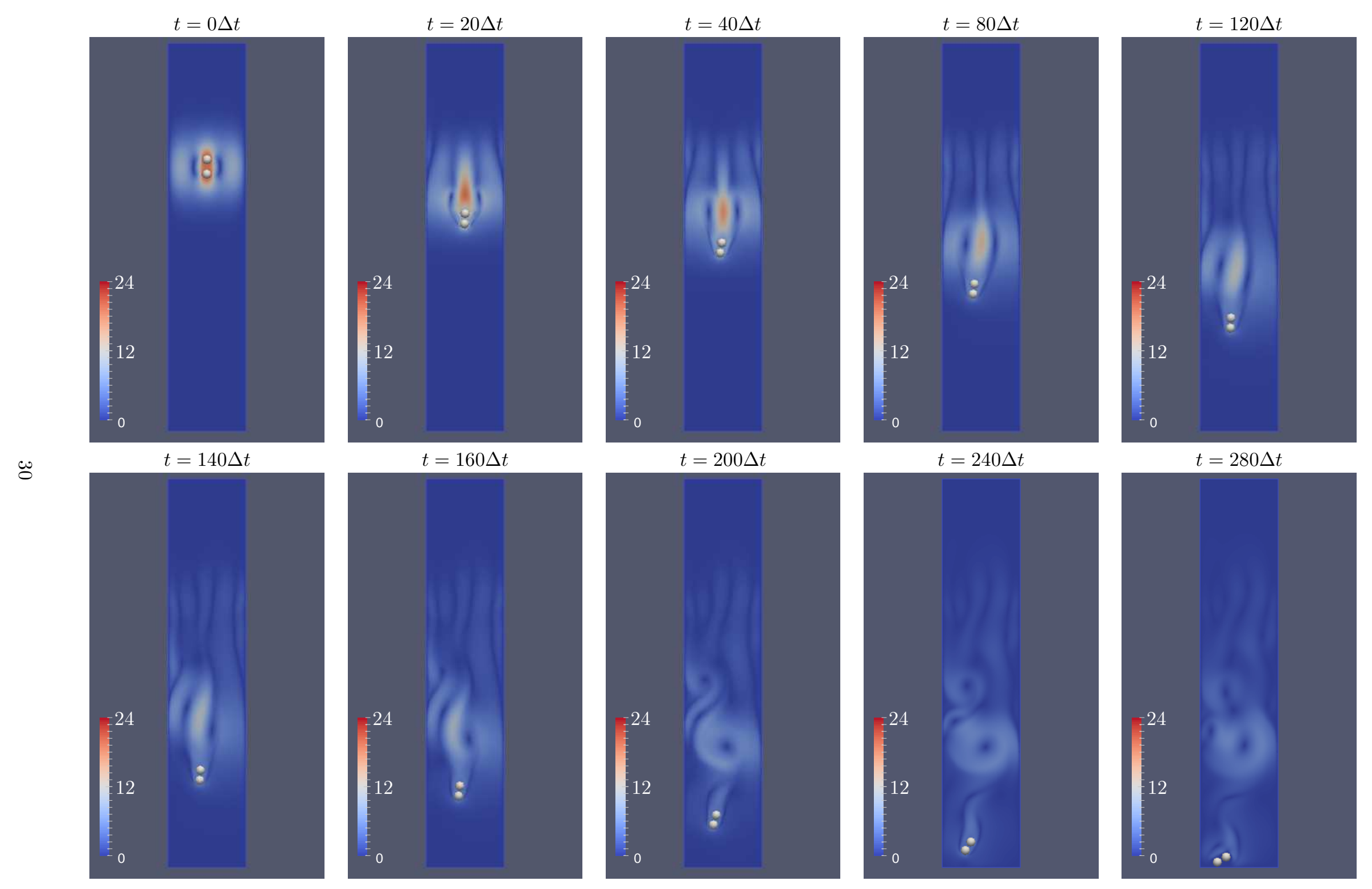

Figure 10: Experiment [B]. Two sedimenting disks in a viscoelastic fluid: modulus $\|\mathbf{u}\|$ of the velocity field. 

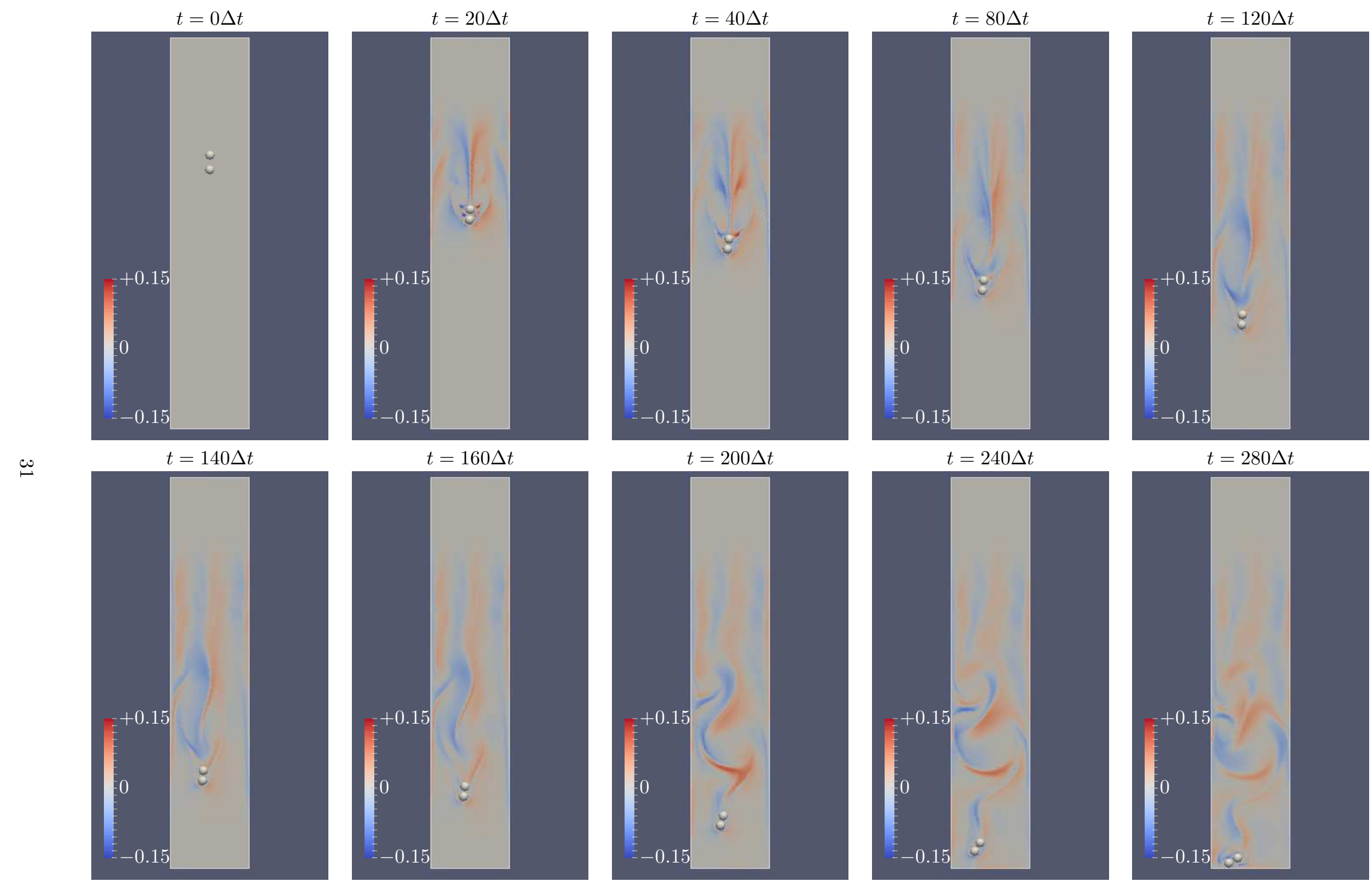

Figure 11: Experiment [B]. Two sedimenting disks in a viscoelastic fluid: shear component $\sigma_{x y}$ of the elastic tensor. 


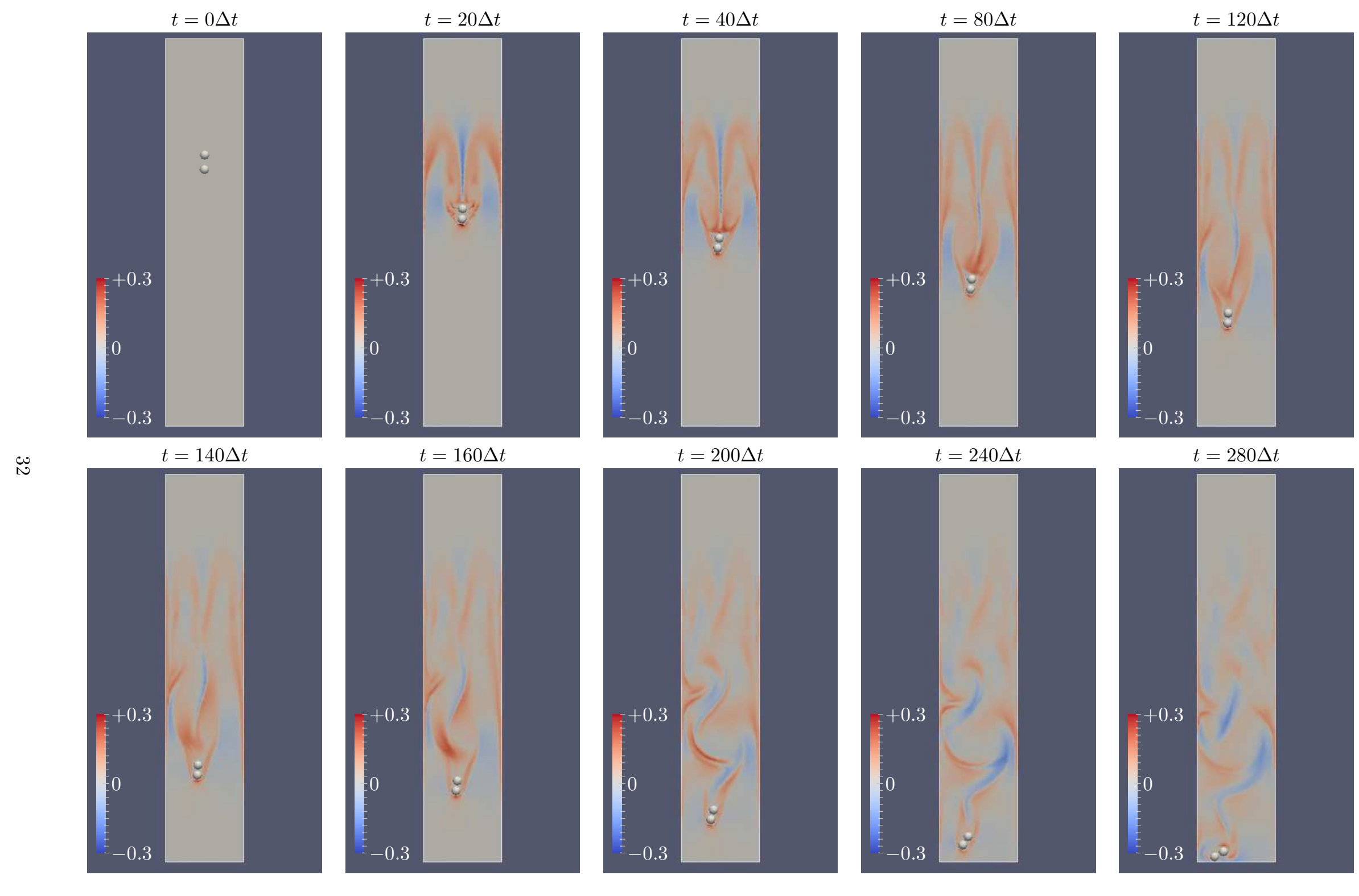

Figure 12: Experiment [B]. Two sedimenting disks in a viscoelastic fluid: elongational component $\sigma_{x x}-\sigma_{y y}$ of the elastic tensor. 


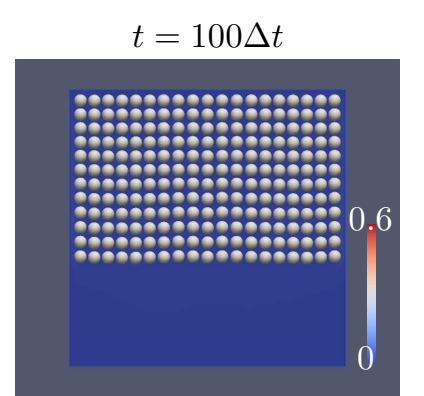

$t=1300 \Delta t$

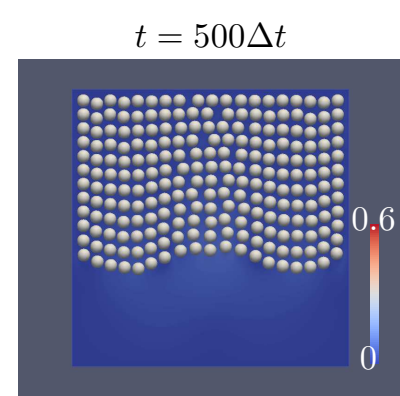

$t=1500 \Delta t$
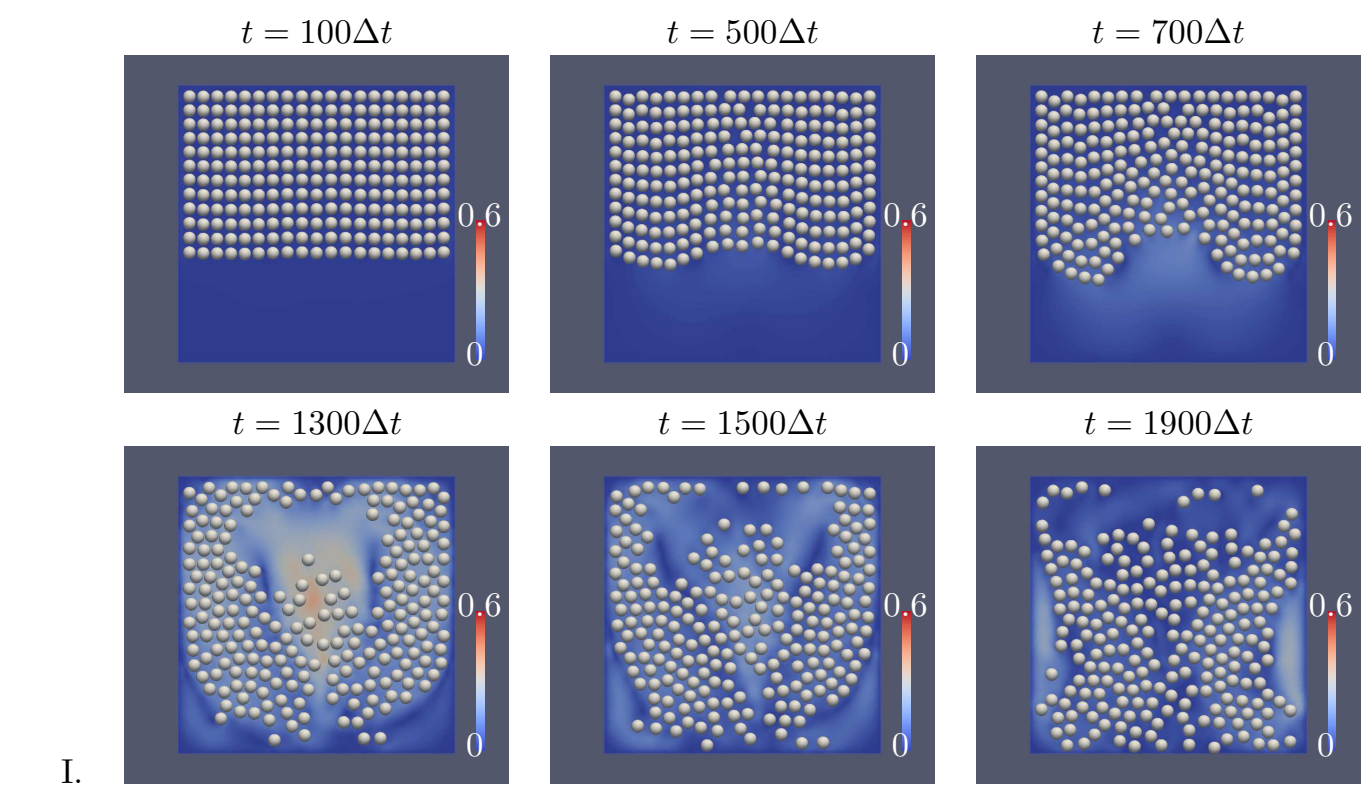

$t=1900 \Delta t$
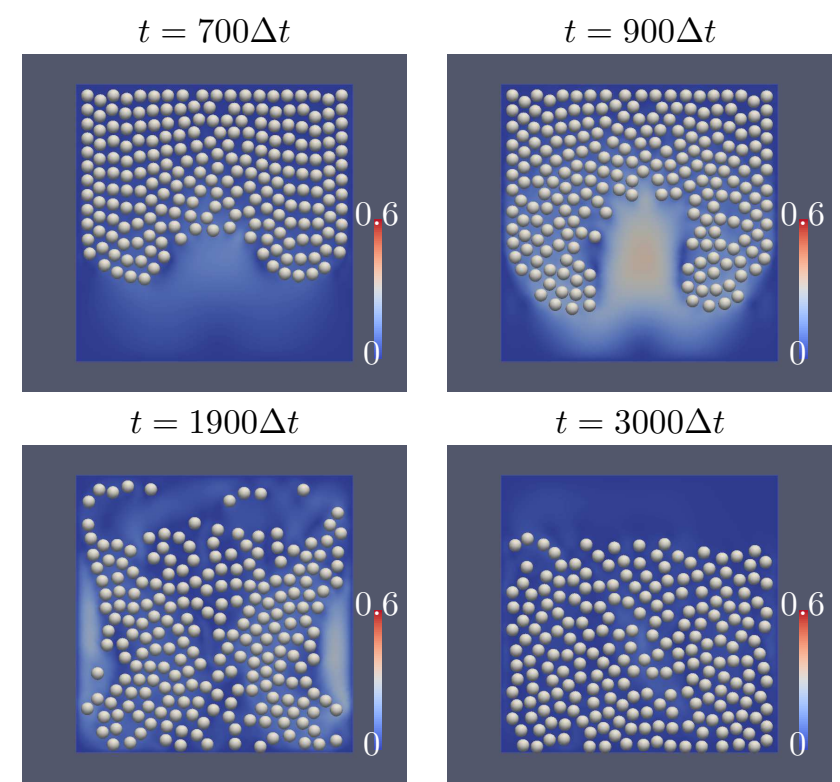

$t=3000 \Delta t$
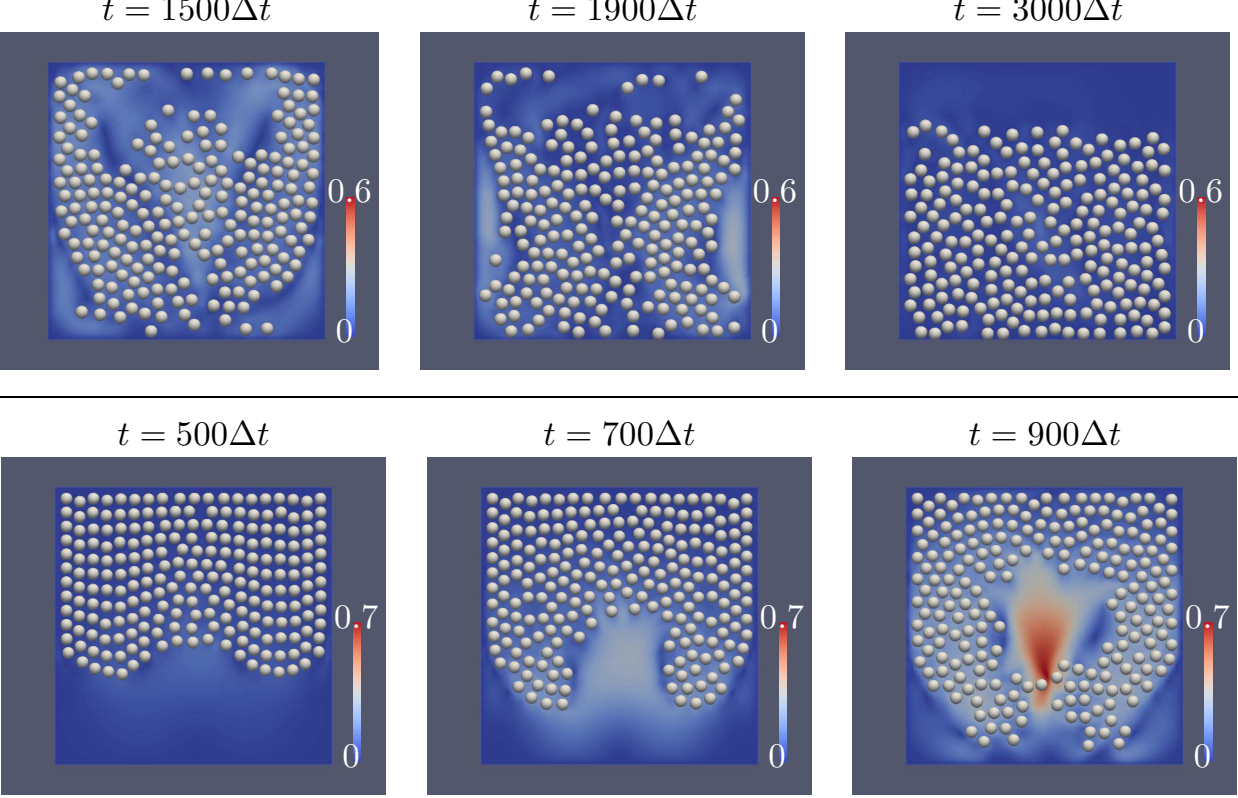

$t=1900 \Delta t$

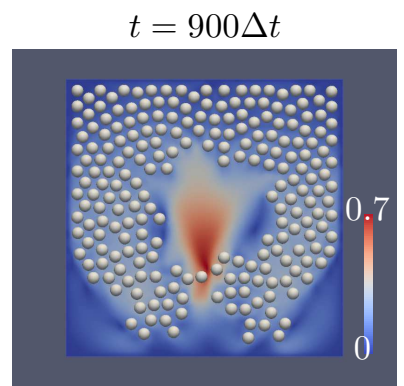

$t=3000 \Delta t$
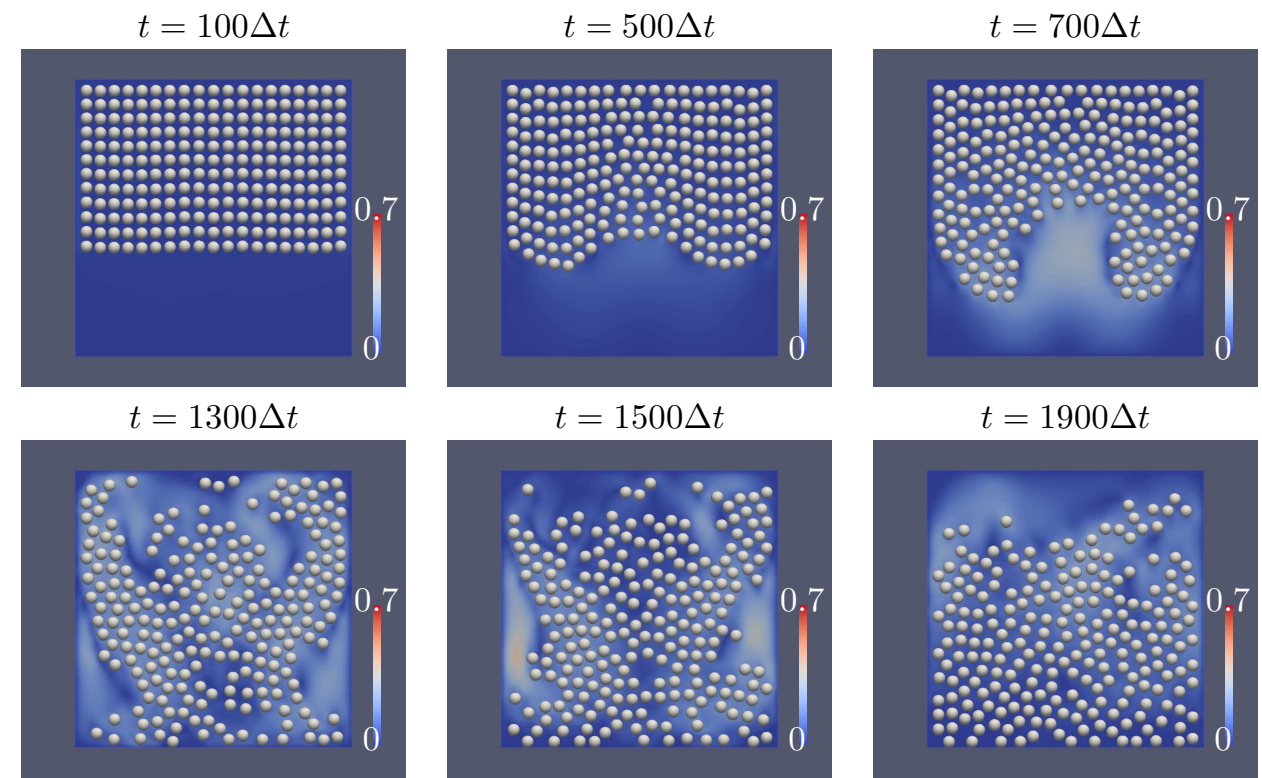

$t=1300 \Delta t$

II.
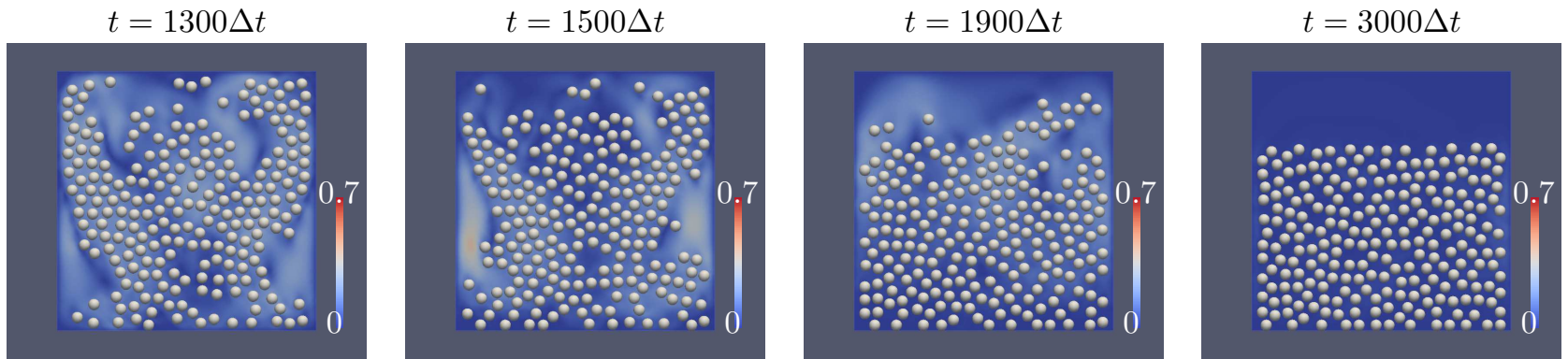

$t=1100 \Delta t$

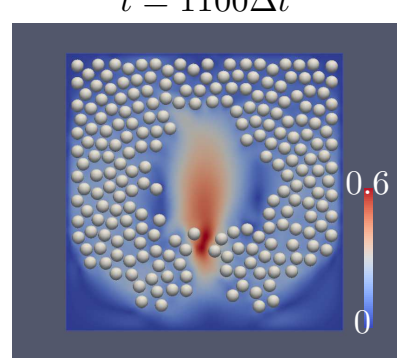

$t=40000 \Delta t$

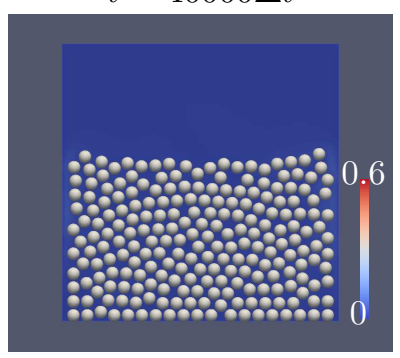

ن.

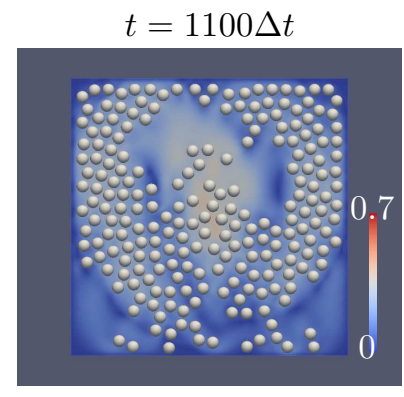

$t=40000 \Delta t$

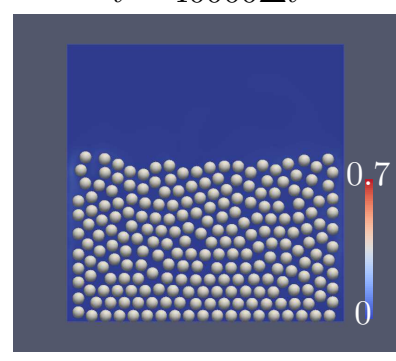

Figure 13: Experiment [C]. Sedimentation of 228 particles and modulus $\|\mathbf{u}\|$ of the velocity field: I) Newtonian fluid, II) viscoelastic fluid. 


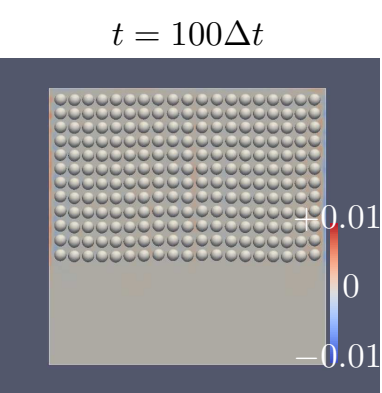

$t=1300 \Delta t$

II.a)

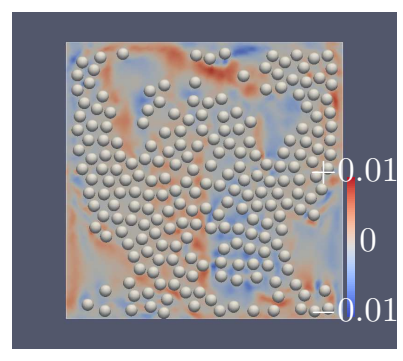

$$
t=100 \Delta t
$$

$t=500 \Delta t$

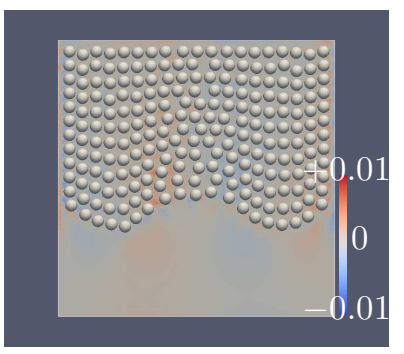

$t=1500 \Delta t$

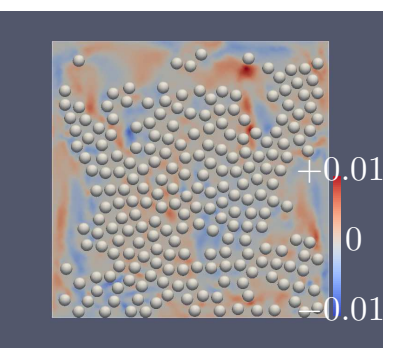

$t=500 \Delta t$

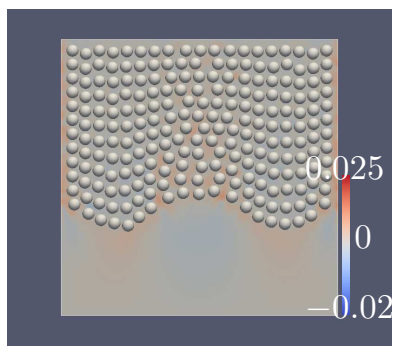

$t=1500 \Delta t$

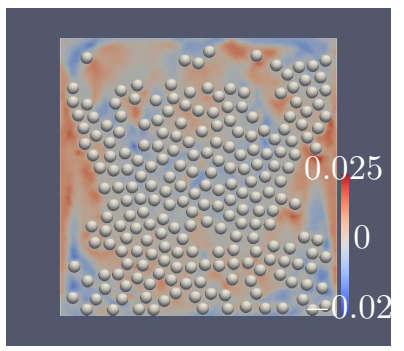

$t=700 \Delta t$

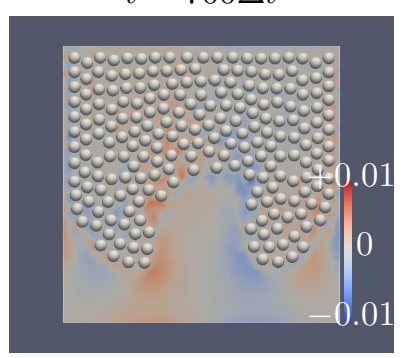

$t=1900 \Delta t$

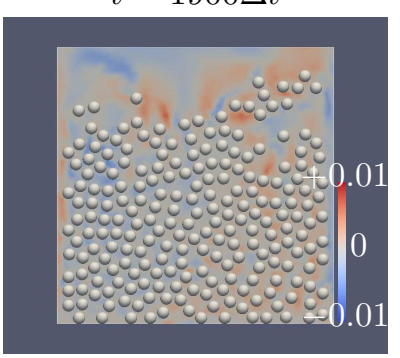

$t=700 \Delta t$

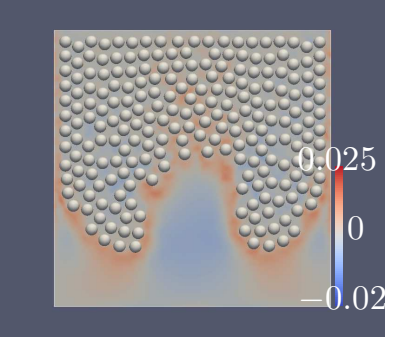

$t=1900 \Delta t$

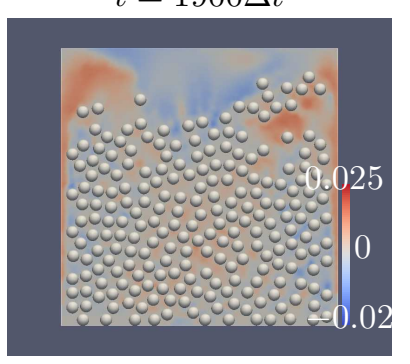

$t=900 \Delta t$

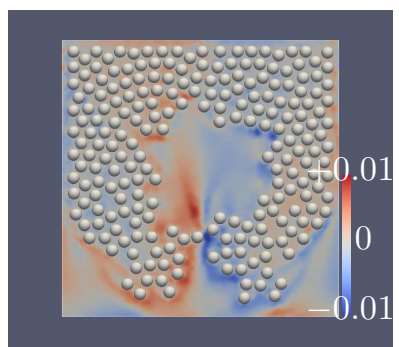

$t=3000 \Delta t$

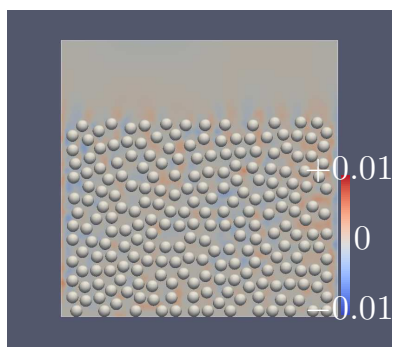

$t=900 \Delta t$

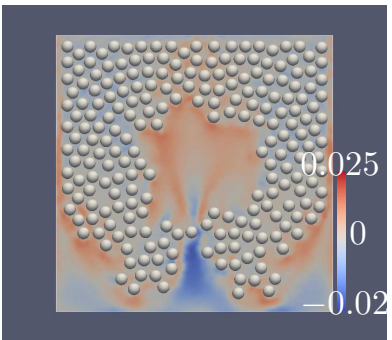

$t=3000 \Delta t$

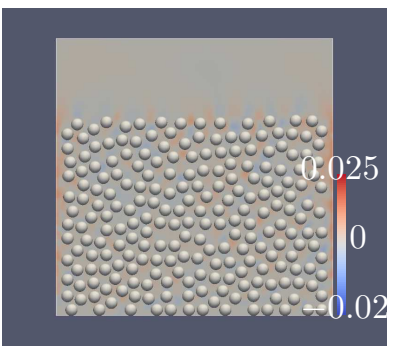

$t=1100 \Delta t$

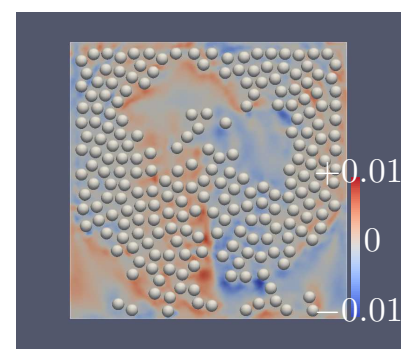

$t=40000 \Delta t$

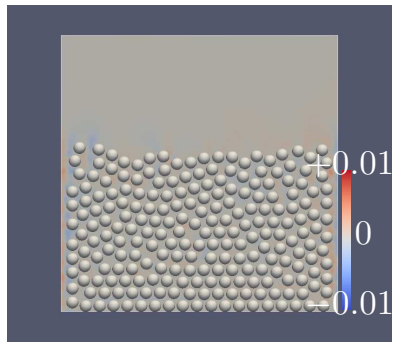

$t=1100 \Delta t$

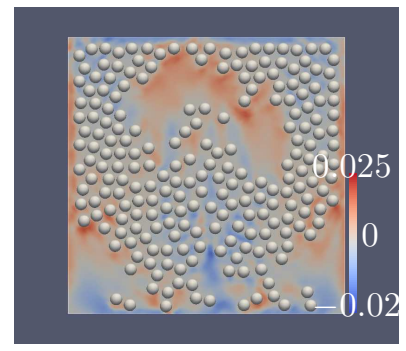

$t=40000 \Delta t$

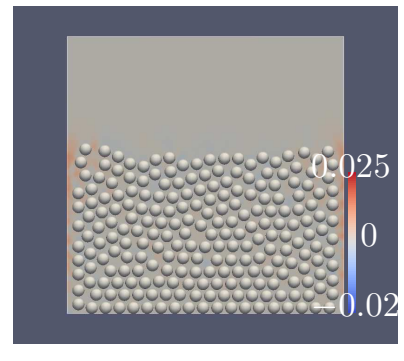

II.b)

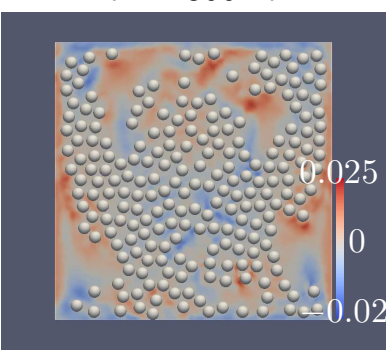

Figure 14: Experiment [C]. Sedimentation of 228 particles in a viscoelastic fluid and: II.a) shear component $\sigma_{x y}$ of the elastic tensor, II.b) elongational component $\sigma_{x x}-\sigma_{y y}$ of the elastic tensor. 


\begin{tabular}{l||c|c|c|c|c|c} 
& $\phi=0.32 \%$ & $\phi=0.96 \%$ & $\phi=1.59 \%$ & $\phi=3.18 \%$ & $\phi=6.37 \%$ & $\phi=12.74 \%$ \\
\hline$\mu_{\text {eff. }}$ & 1.0083 & 1.0247 & 1.0421 & 1.0858 & 1.1819 & 1.4391 \\
$\mu_{\text {eff.,th. }}=\mu(1+2 \phi)$ & 1.0064 & 1.0191 & 1.0318 & 1.0637 & 1.1274 & 1.2547 \\
$\mathcal{E}:=\frac{\left|\mu_{\text {eff. }}-\mu_{\text {eff.,th. }}\right|}{\mu_{\text {eff.,th. }}}$ & $0.19 \%$ & $0.54 \%$ & $1.00 \%$ & $2.08 \%$ & $4.84 \%$ & $14.69 \%$
\end{tabular}

Table 3: Experiment [D]. Effective viscosity of a suspension of circular particles in a Newtonian fluid. Comparison between the numerical result and the theoretical result for various values of the solid fraction $\phi$. Here, $\mathcal{E}=\frac{\left|\mu_{\text {eff. }}-\mu_{\text {eff.,th. }}\right|}{\mu_{\text {eff.,th. }}}$ measures the relative distance between the numerical value $\mu_{\text {eff. }}$ and Einstein theoretical value $\mu_{\text {eff.,th. }}$ of the effective viscosity.

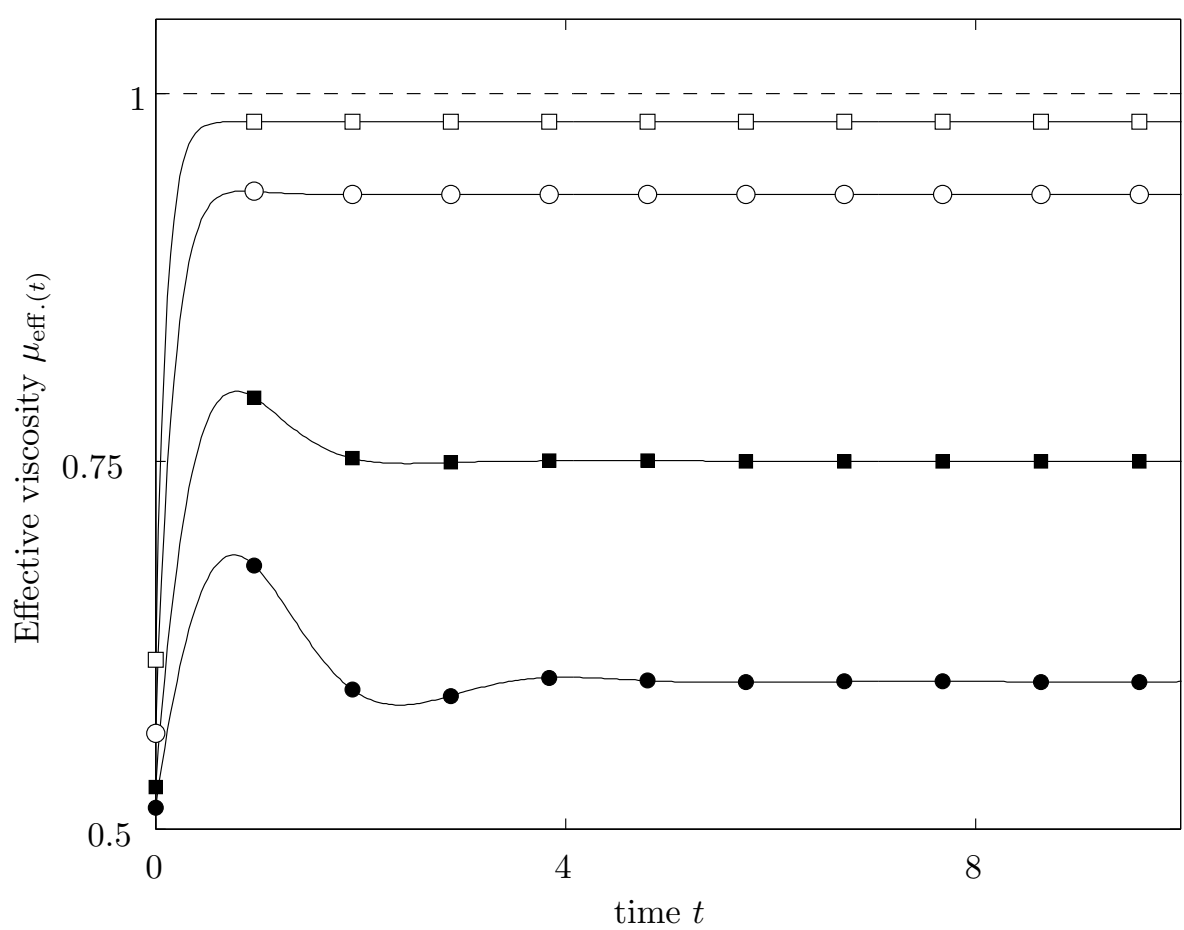

Figure 15: Experiment [E]. Effective viscosity of a viscoelastic fluid with $r=0.50$ and different values of $\lambda$. Newtonian case is represented as $\lambda=0.00(-)$; viscoelastic cases are represented as $\lambda=0.10(\square), \lambda=0.20(0), \lambda=0.50(\square)$, $\lambda=1.00(\bullet)$. 

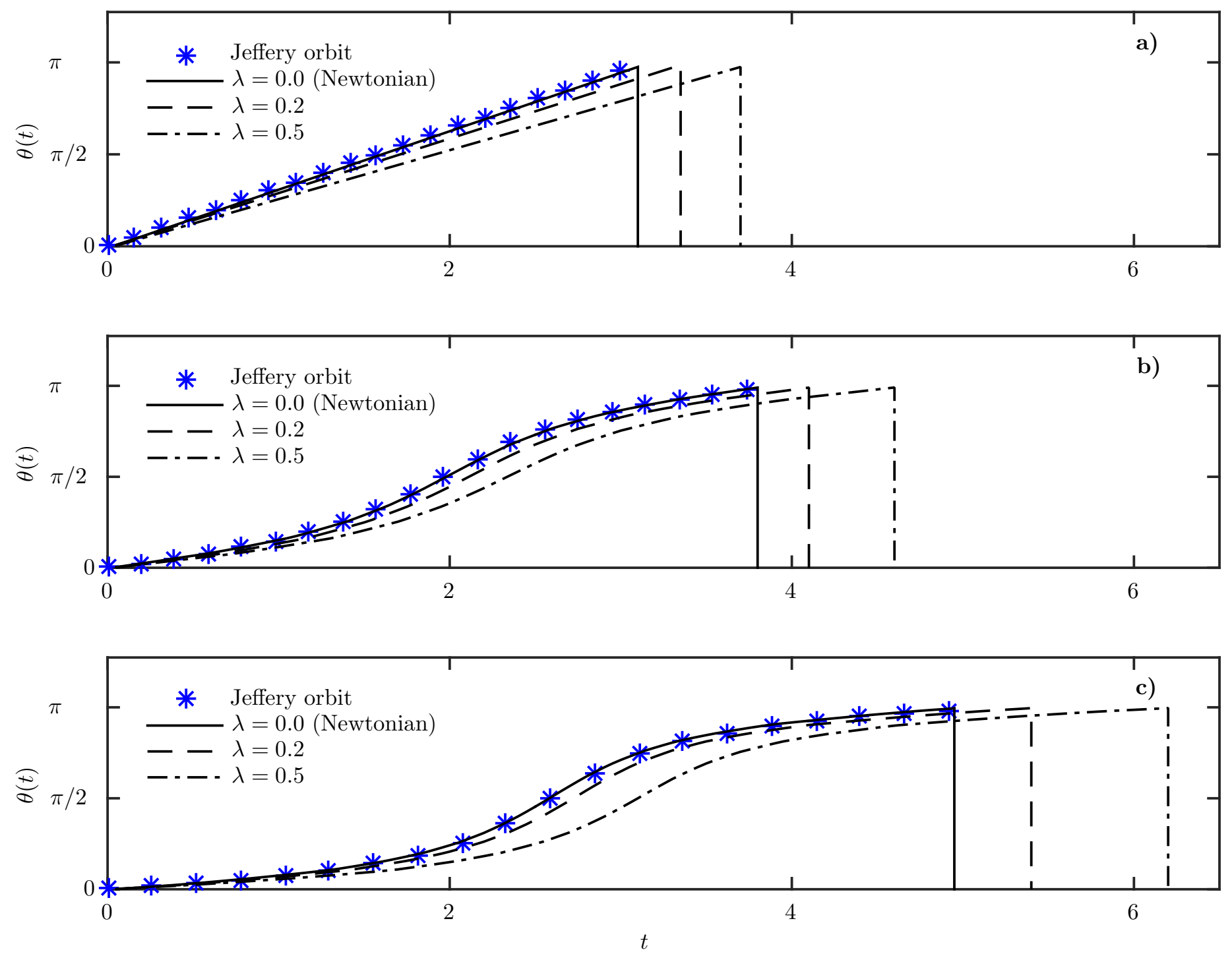

Figure 16: Experiment $\left[\mathrm{F}_{1}\right]$. Jeffery orbits of an ellipsoïd in a viscoelastic fluid, for various eccentricity values. Time scales over a half-period of revolution. The semi-axes are denoted $R_{i}$ and the product $R_{1} R_{2}$ is assumed to be constant: a) $R_{1} / R_{2}=1$ (disk), b) $R_{1} / R_{2}=2$, c) $R_{1} / R_{2}=3$. 

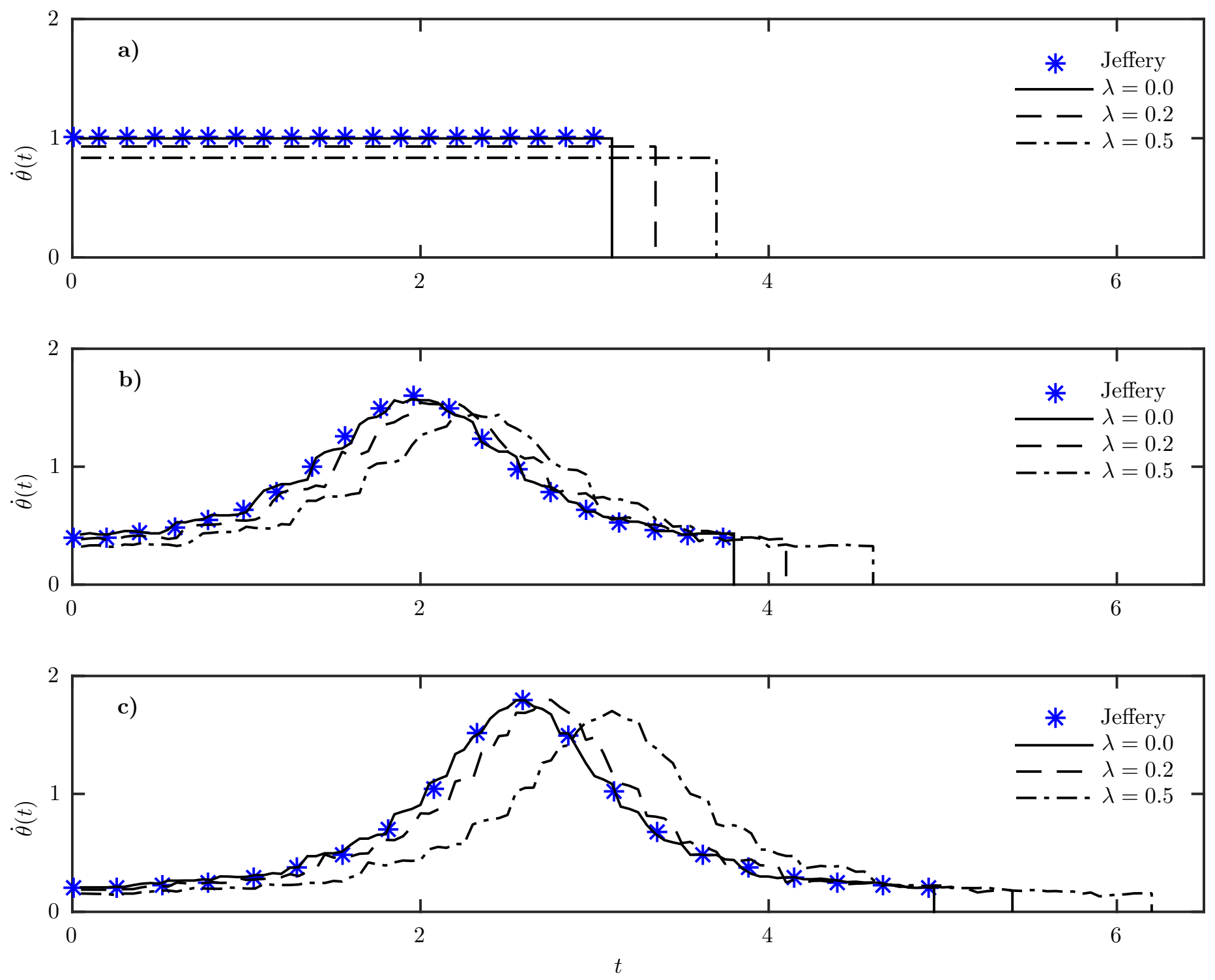

Figure 17: Experiment $\left[\mathrm{F}_{1}\right]$. Angular velocity of an ellipsoïd in a viscoelastic fluid, for various eccentricity values. Time scales over a half-period of revolution. The semi-axes are denoted $R_{i}$ and the product $R_{1} R_{2}$ is assumed to be constant: a) $R_{1} / R_{2}=1$ (disk), b) $R_{1} / R_{2}=2$, c) $R_{1} / R_{2}=3$. 

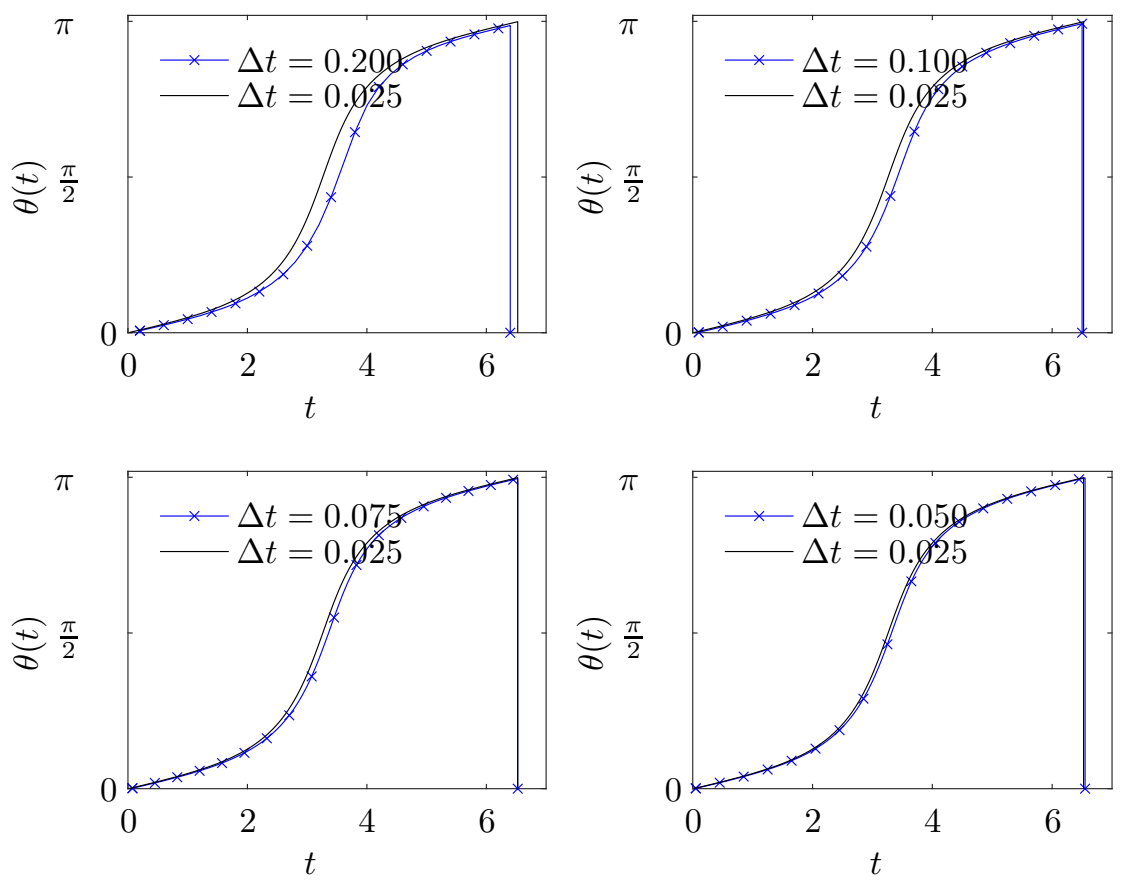

Figure 18: Influence of the time step $\Delta t$ on the dynamics of an ellipse in a shear flow in a viscoelastic case.
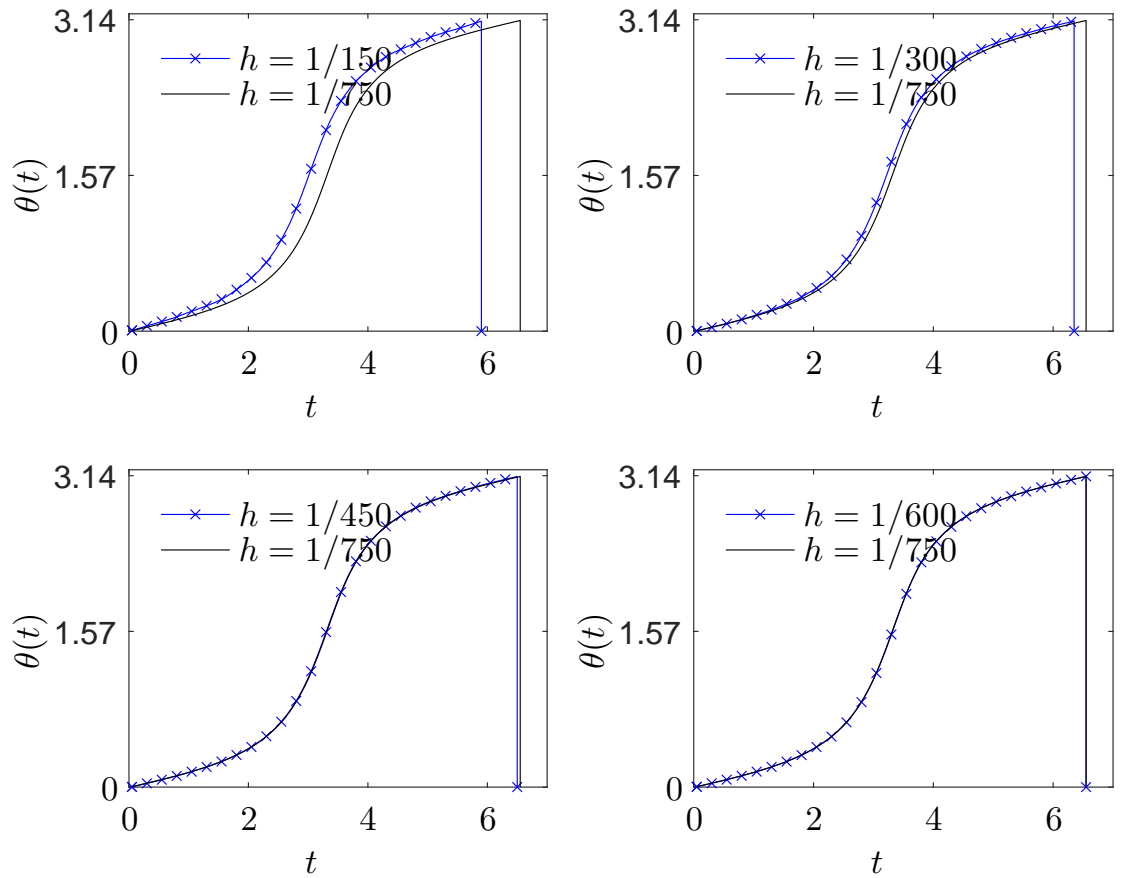

Figure 19: Influence of the mesh size $h$ on the dynamics of an ellipse in a shear flow in a viscoelastic case. 

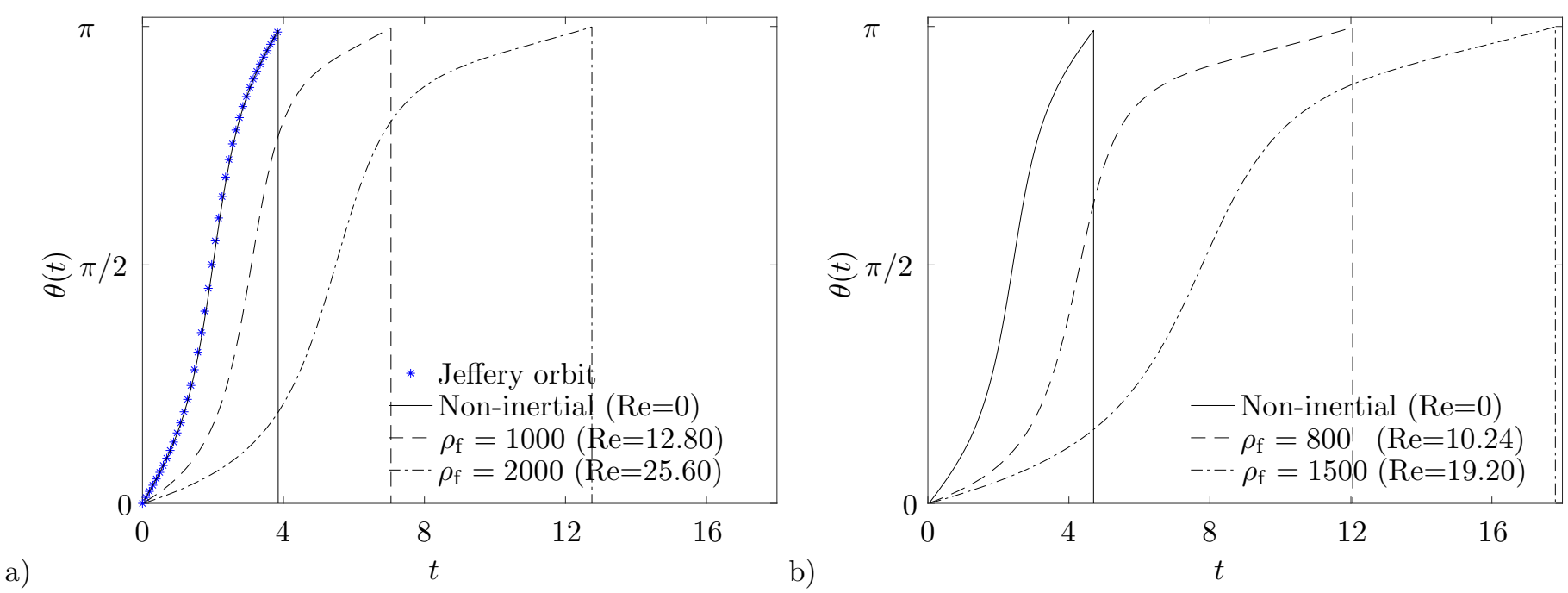

Figure 20: Experiment $\left[\mathrm{F}_{2}\right]$. Angular orbit of an ellipse with ratio $R_{1} / R_{2}=2$ for different values of the Reynolds number. a) Newtonian fluid $(r=0.0, \lambda=0.0)$, b) viscoelastic fluid $(r=0.5, \lambda=0.5)$. 


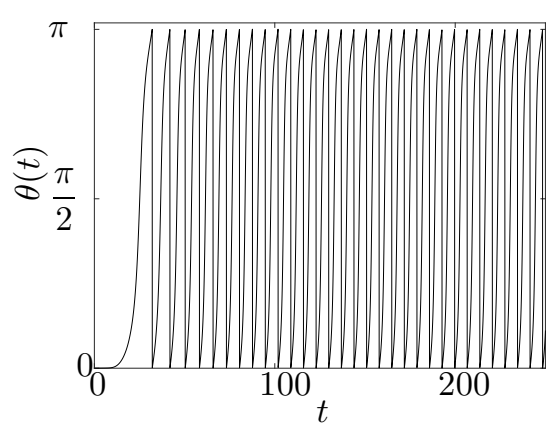

$\mathrm{Re}=12.80$

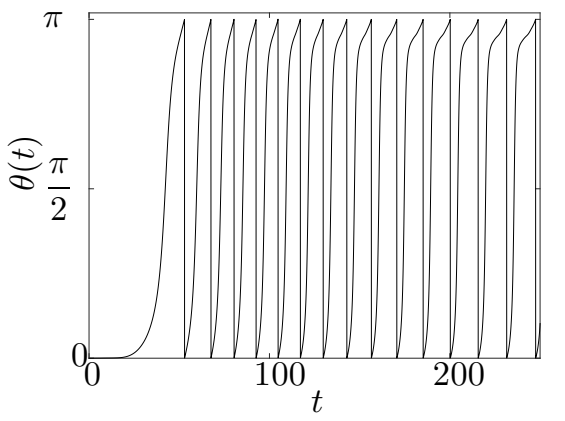

$\mathrm{R} e=25.60$

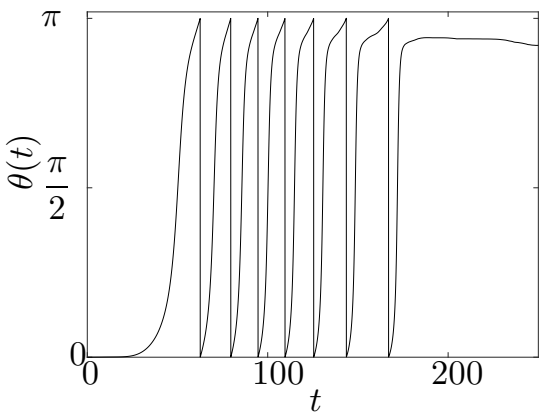

$\mathrm{R} e=32.00$

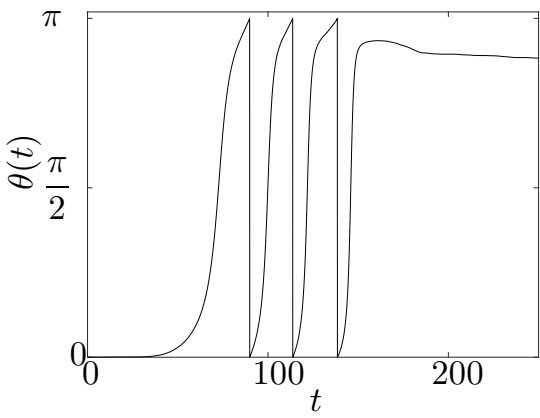

$\mathrm{R} e=51.20$

Figure 21: Experiment $\left[\mathrm{F}_{2}\right]$. Behaviour of an ellipse with ratio $R_{1} / R_{2}=2$ in a Newtonian fluid (parameters: $r=0.0, \lambda=0.0$ ). Evolution of the orientation angle for various inertial regimes: $\rho_{\mathrm{f}}=1000,2000,2500,4000$.

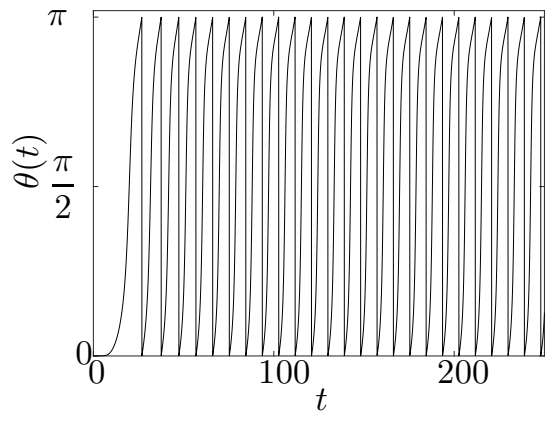

$\mathrm{R} e=6.40$

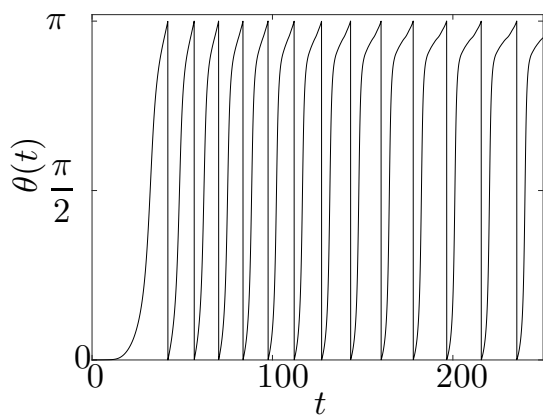

$\mathrm{R} e=12.80$

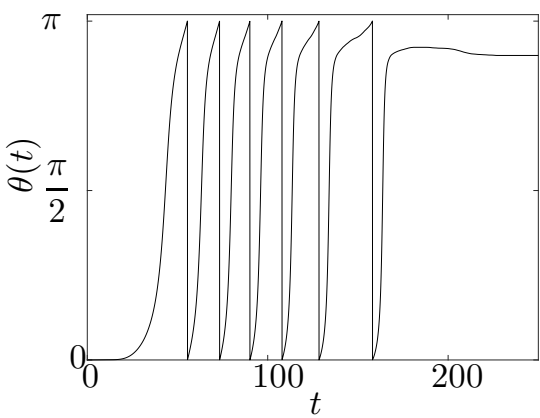

$\mathrm{R} e=19.20$

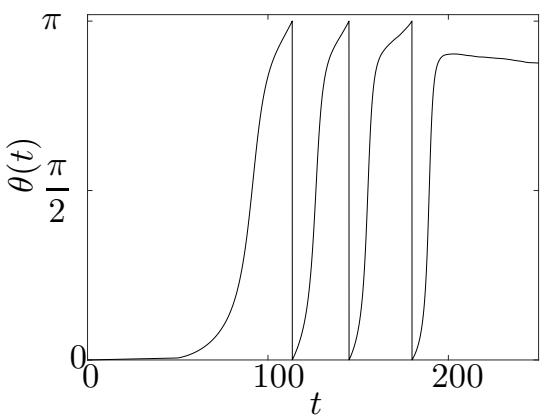

$\mathrm{R} e=51.20$

Figure 22: Experiment $\left[\mathrm{F}_{2}\right]$. Behaviour of an ellipse with ratio $R_{1} / R_{2}=2$ in a viscoelastic fluid (parameters: $r=0.5, \lambda=0.5$ ). Evolution of the orientation angle for various inertial regimes: $\rho_{\mathrm{f}}=500,1000,1500,4000$. 

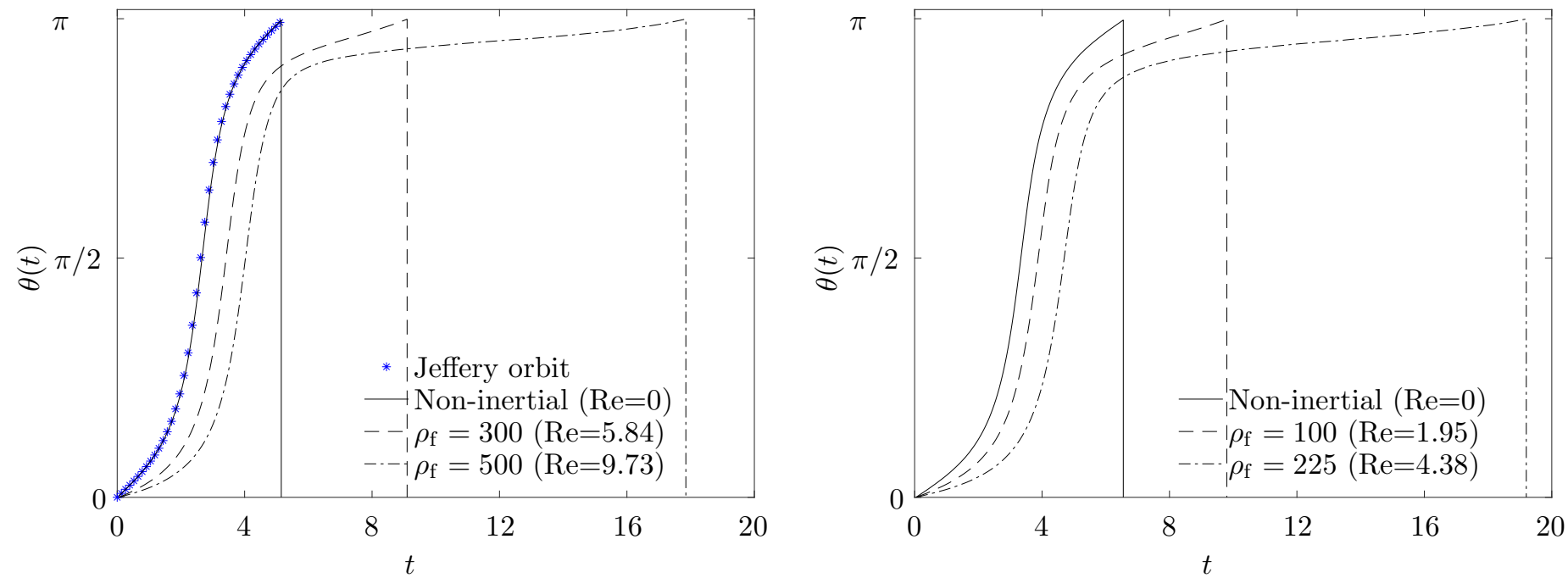

Figure 23: Experiment $\left[\mathrm{F}_{2}\right]$. Angular orbit of an ellipse with ratio $R_{1} / R_{2}=3$ for different values of the Reynolds number. a) Newtonian fluid $(r=0.0, \lambda=0.0)$, b) viscoelastic fluid $(r=0.5, \lambda=0.5)$. 

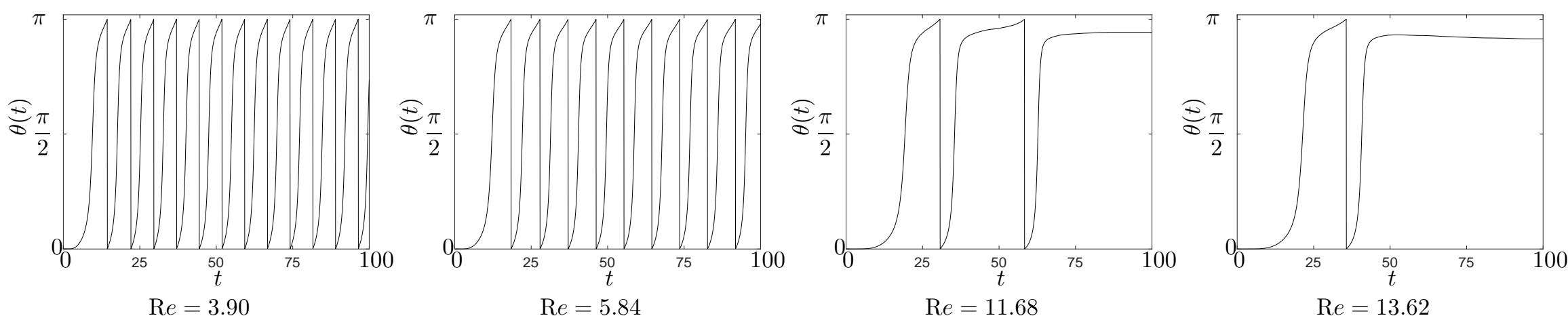

Figure 24: Experiment $\left[\mathrm{F}_{2}\right]$. Behaviour of an ellipse with ratio $R_{1} / R_{2}=3$ in a Newtonian fluid (parameters: $r=0.0, \lambda=0.0$ ). Evolution of the orientation angle for various inertial regimes: $\rho_{\mathrm{f}}=200,300,600,700$.
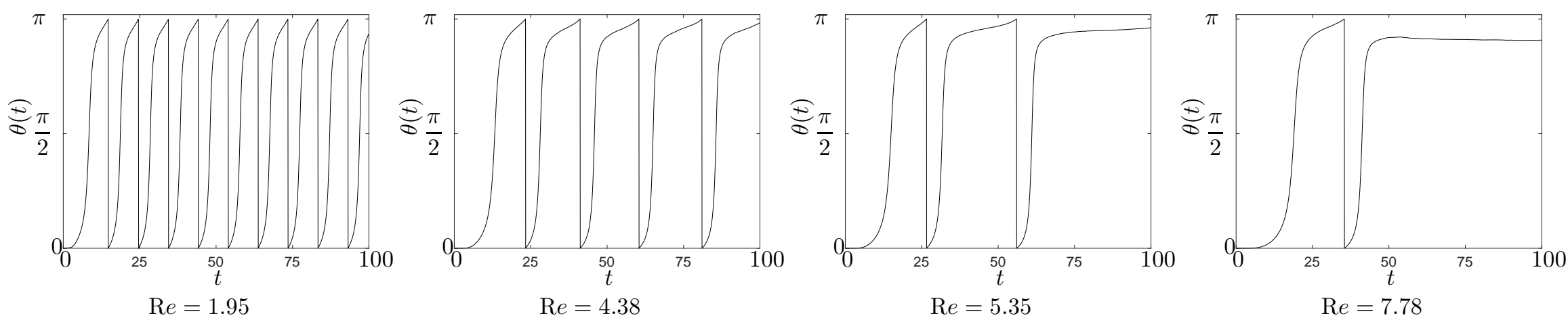

Figure 25: Experiment $\left[\mathrm{F}_{2}\right]$. Behaviour of an ellipse with ratio $R_{1} / R_{2}=3$ in a viscoelastic fluid (parameters: $r=0.5, \lambda=0.5$ ). Evolution of the orientation angle for various inertial regimes: $\rho_{\mathrm{f}}=100,225,275,400$. 
$t=0 \Delta t$

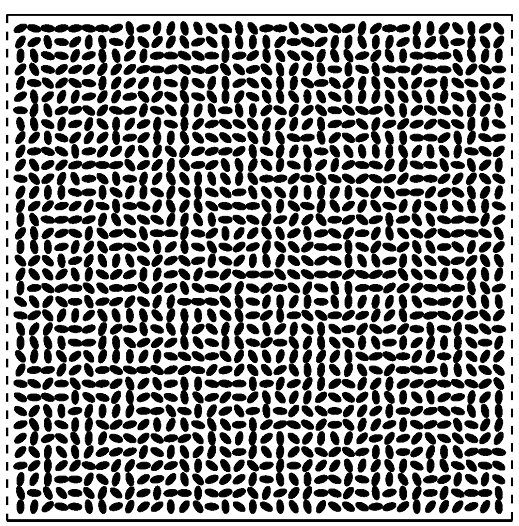

$t=40 \Delta t$

记

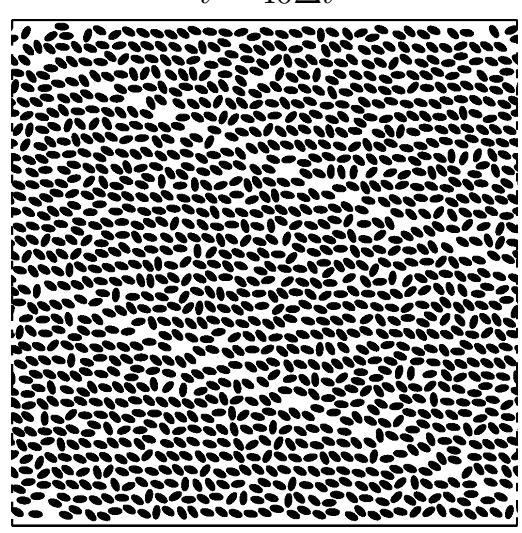

$t=10 \Delta t$

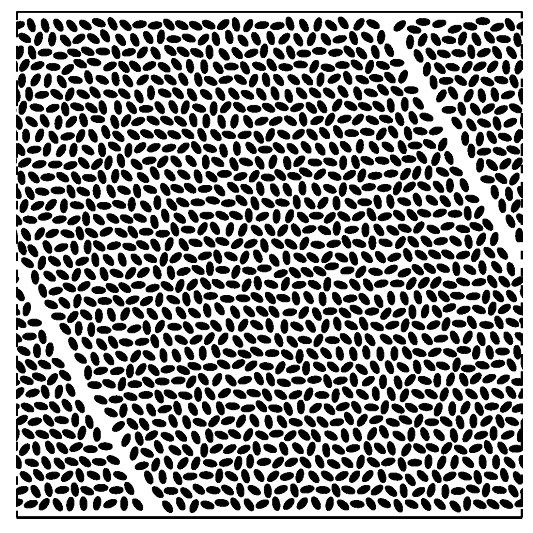

$t=50 \Delta t$

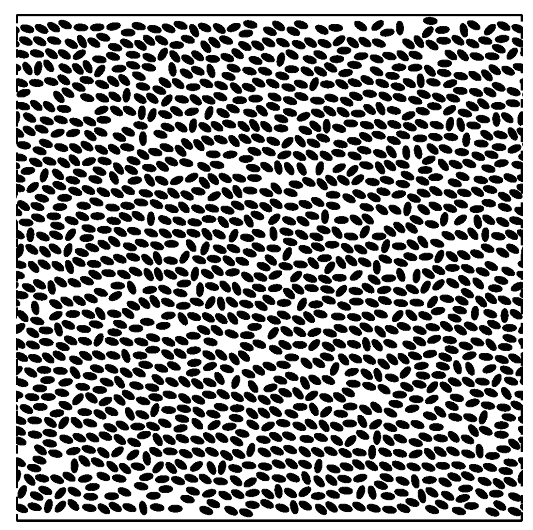

$t=20 \Delta t$

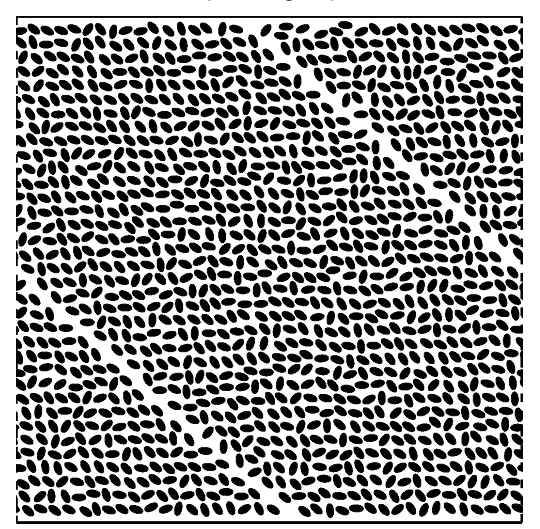
$t=60 \Delta t$

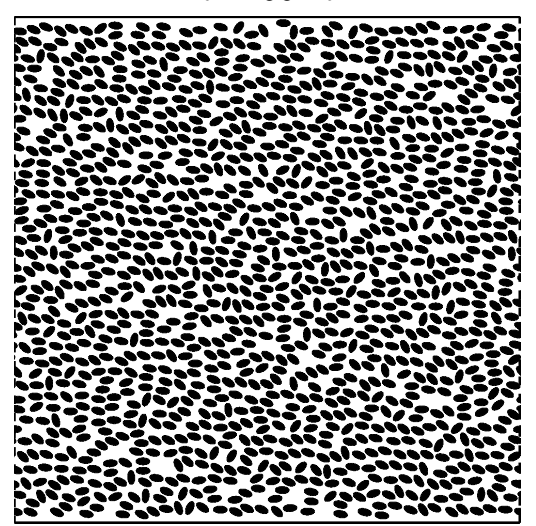

$t=30 \Delta t$

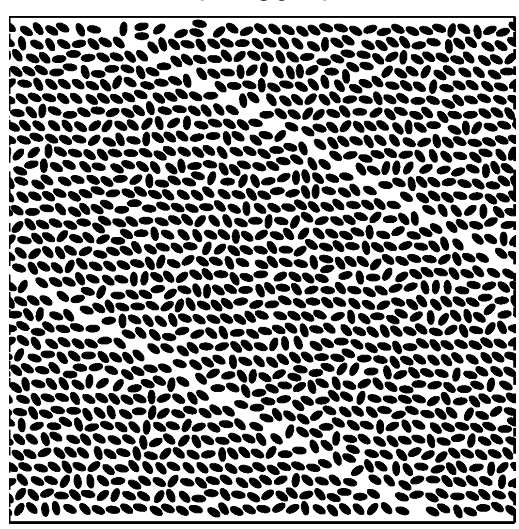

$t=250 \Delta t$

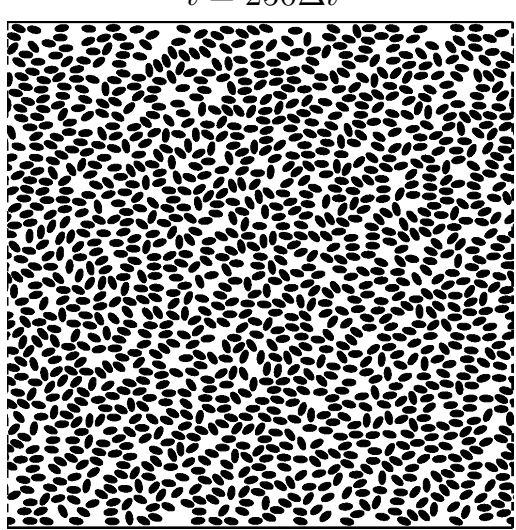

Figure 26: Experiment [G]. Shear of 1296 rigid ellipsoids (solid fraction $\sim 36 \%$ ) in a viscoelastic fluid. Parameters: $r=0.50, \lambda=1.00$. 


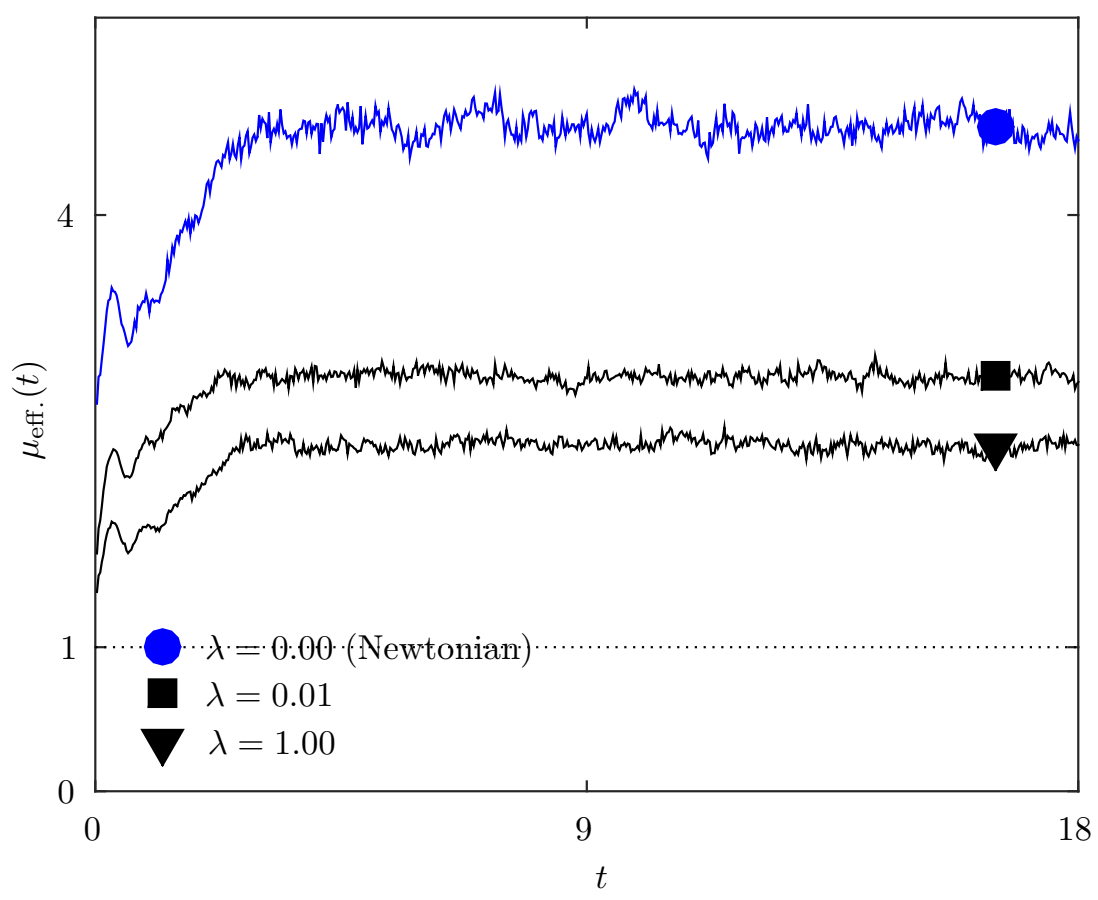

Figure 27: Experiment [G]. Effective viscosity of a suspension of 1296 rigid ellipsoids (solid fraction $\sim 36 \%$ ) with interpolation parameter $r=0.5$ and different values of the elastic relaxation time: $\lambda=0.00$ (Newtonian case), $\lambda=0.01$ and $\lambda=1.00$. 


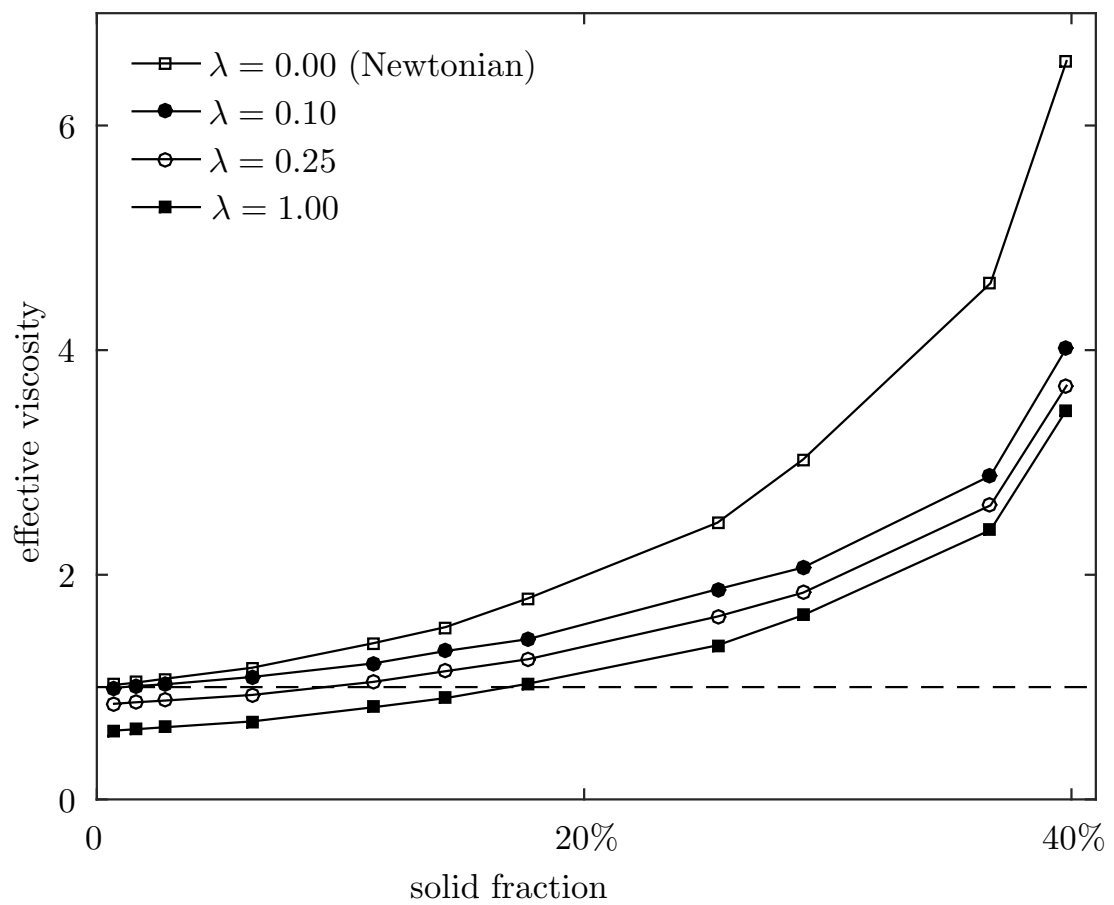

Figure 28: Experiment [G]. Influence of the solid fraction over the effective viscosity in a suspension of rigid ellipsoids in a viscoelastic fluid of Oldroyd type.

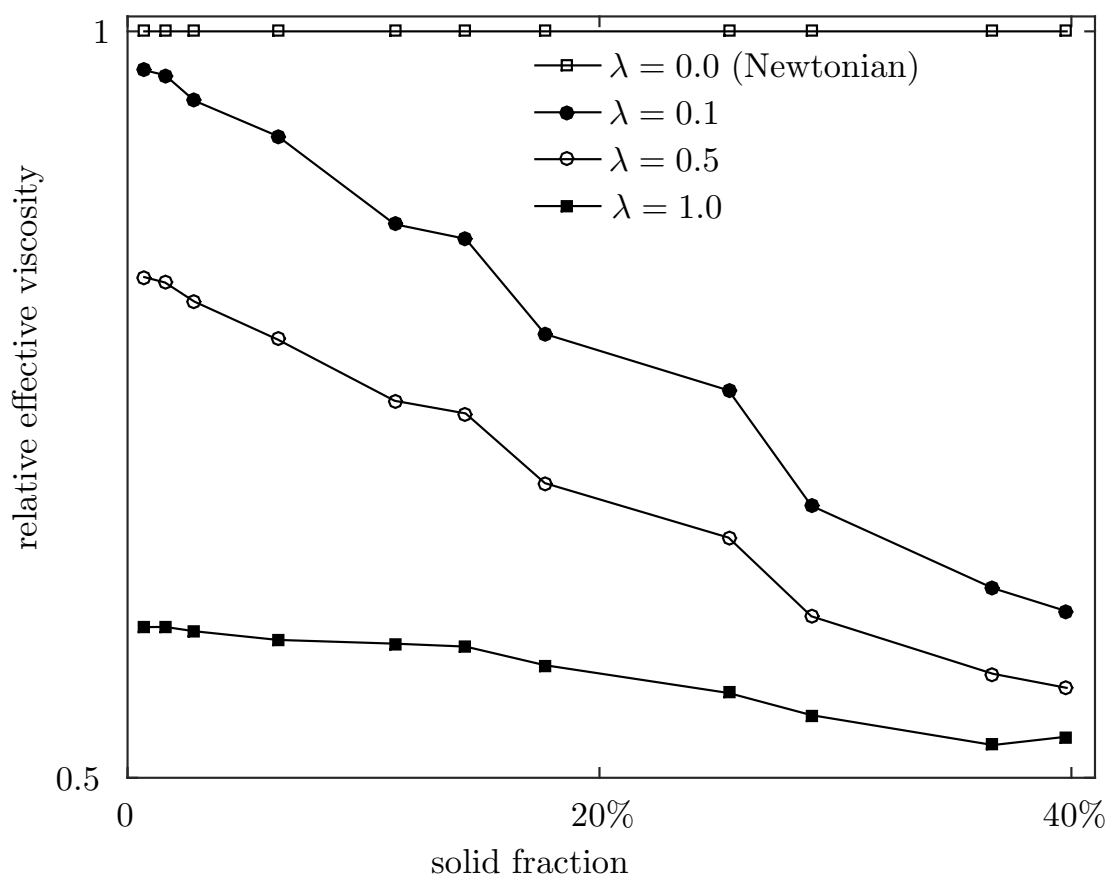

Figure 29: Experiment $[\mathrm{G}]$. Influence of the solid fraction over the relative effective viscosity in a suspension of rigid ellipsoids in a viscoelastic fluid of Oldroyd type. 

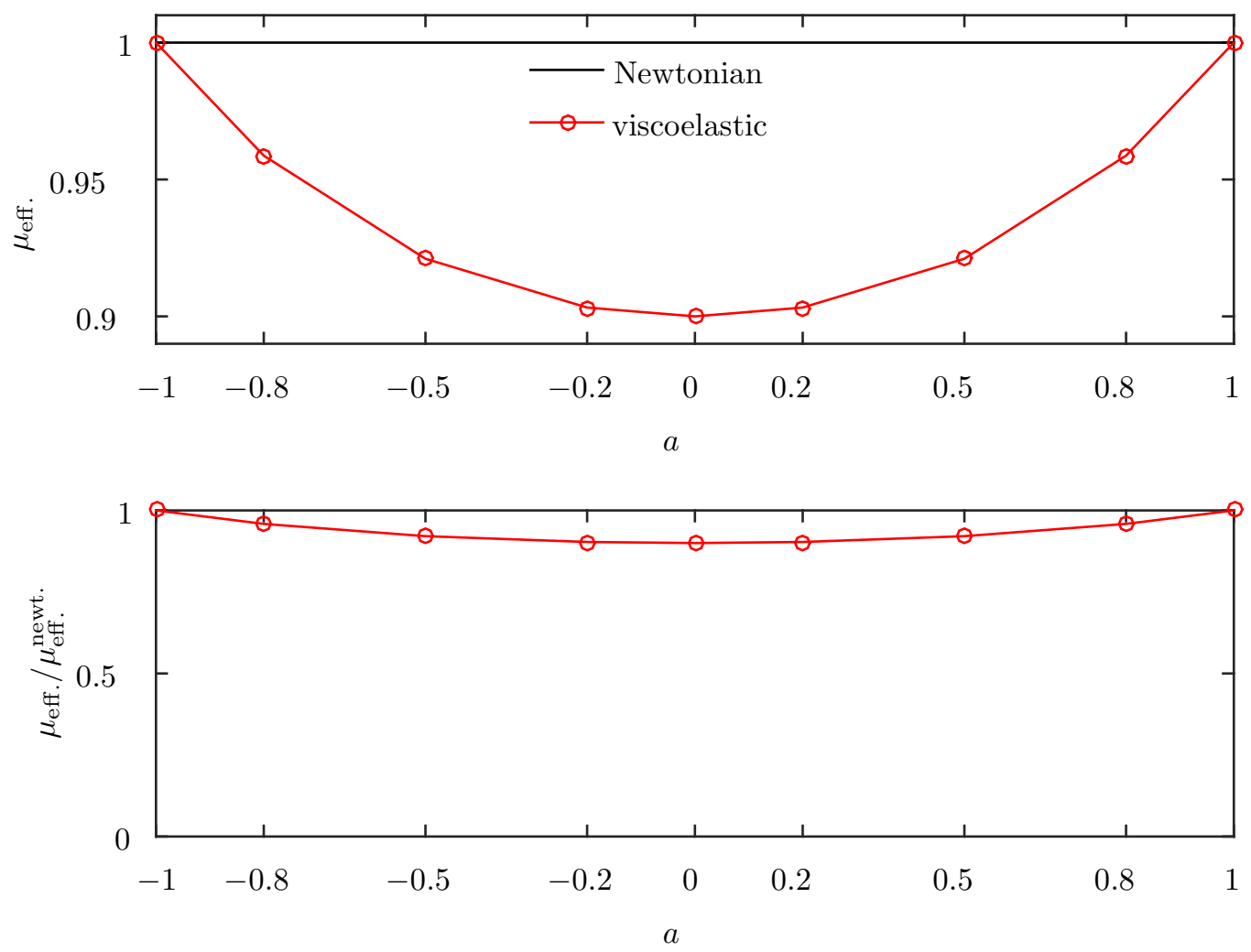

Figure 30: Experiment $[\mathrm{H}]$. Influence of the derivative parameter $a \in(-1,1)$ over the effective viscosity for a suspension driven by the Johnson-Segalman model. Parameters: $N=0$ (number of rigid entities), $r=0.5$ (interpolation parameter), $\lambda=0.25$ (elastic relaxation time). 

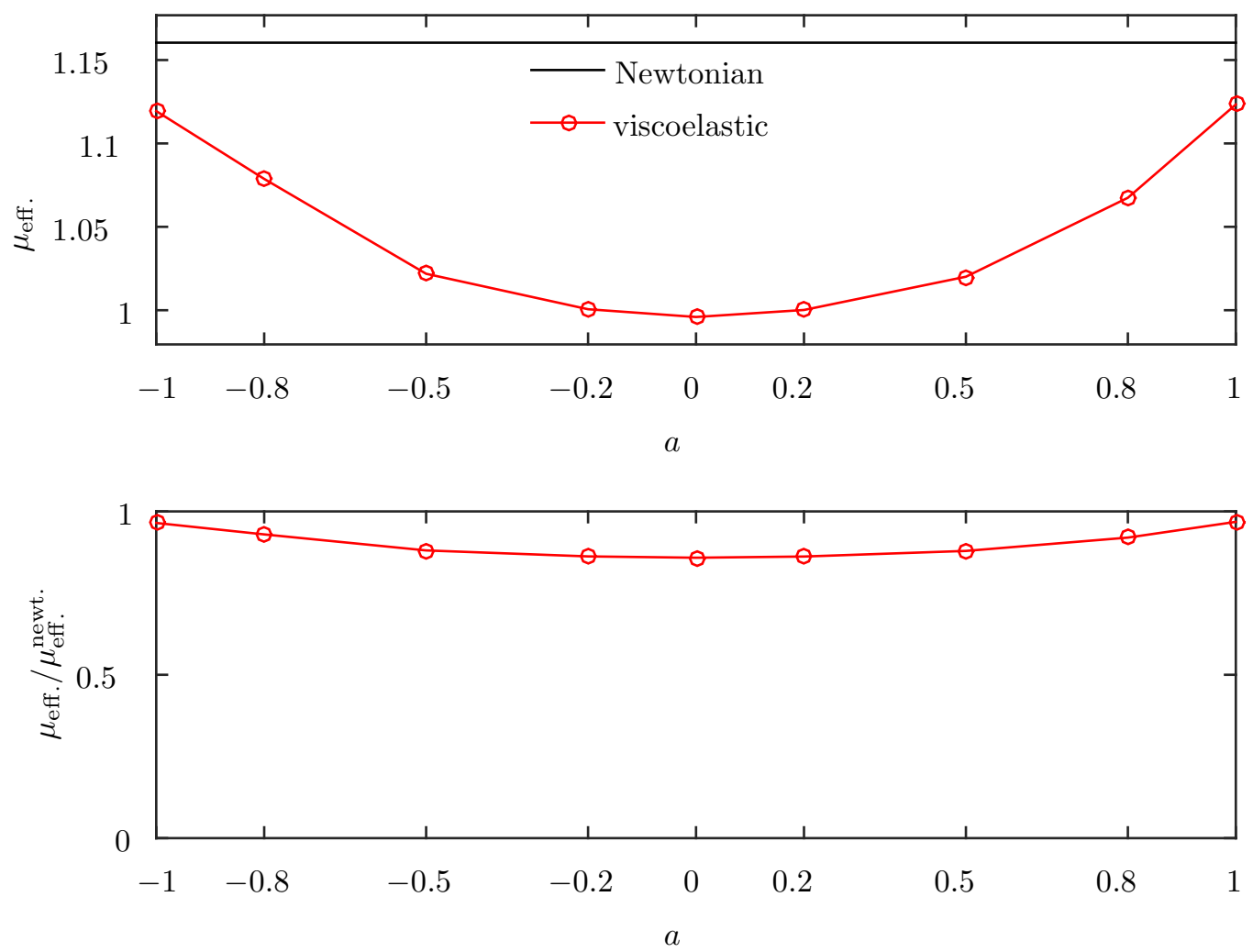

Figure 31: Experiment $[\mathrm{H}]$. Influence of the derivative parameter $a \in(-1,1)$ over the effective viscosity for a suspension driven by the Johnson-Segalman model. Parameters: $N=100$ (number of rigid entities), $r=0.5$ (interpolation parameter), $\lambda=0.25$ (elastic relaxation time). 

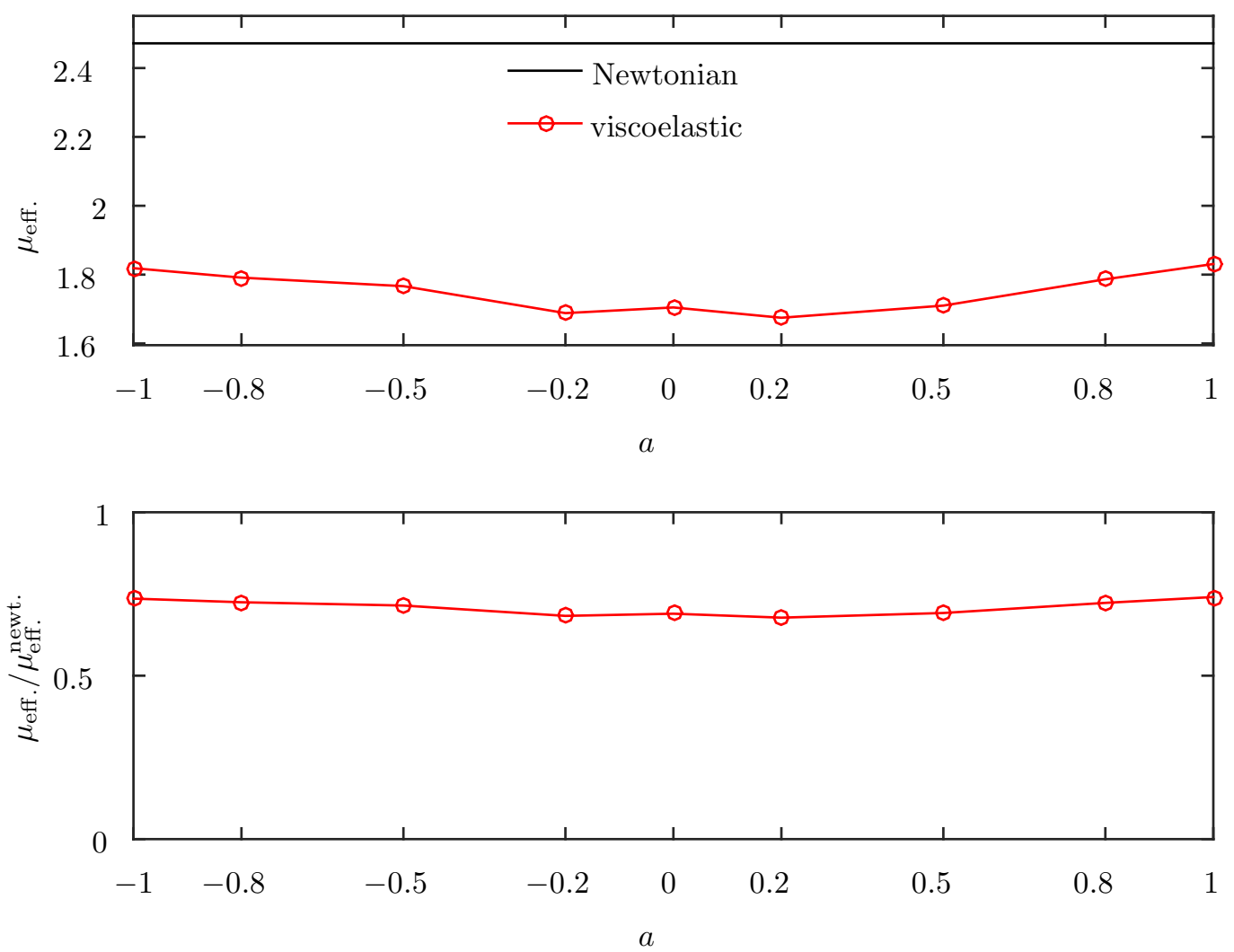

Figure 32: Experiment $[\mathrm{H}]$. Influence of the derivative parameter $a \in(-1,1)$ over the effective viscosity for a suspension driven by the Johnson-Segalman model. Parameters: $N=400$ (number of rigid entities), $r=0.5$ (interpolation parameter), $\lambda=0.25$ (elastic relaxation time). 